TE WHARE WĀNANGA O TE ŪPOKO O TE IKA A MĀUI

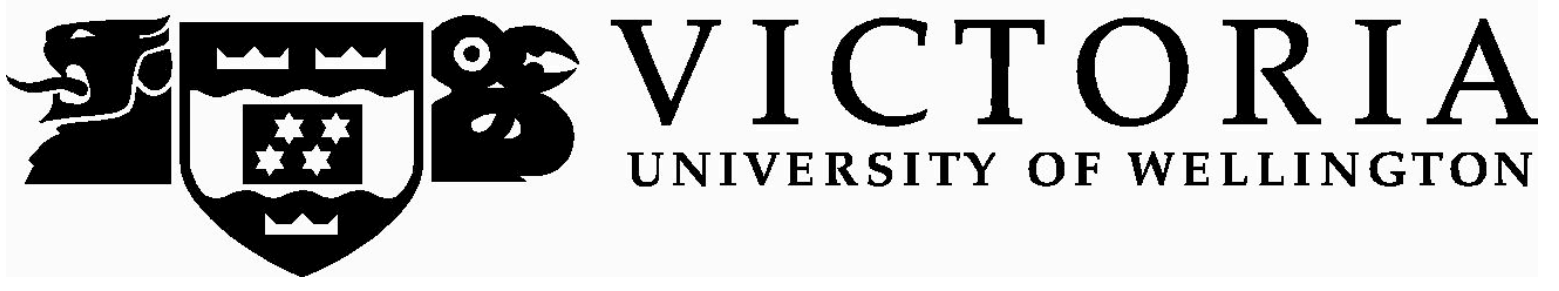

School of History, Philosophy, Political Science and International Relations

\title{
CAVE ART AND THE EVOLUTION OF THE HUMAN MIND
}

\author{
MARTIN PAUL GRAY
}

A thesis submitted to Victoria University of Wellington in fulfilment of the requirements for the degree of Master of Arts in Philosophy 
For Mum and Dad

The more I learn, the less I seem to know... 


\begin{abstract}
The discovery of cave paintings made by our Upper Paleolithic ancestors in Western Europe was an astonishing find - so astonishing, that they were originally believed to have been fakes. However, as more sites were uncovered, their authenticity was confirmed. But how could these people, who at the time of the discovery were believed to be merely dumb brutes, create such beautiful and naturalistic representations? And an even more difficult question to answer was, why?
\end{abstract}

In this thesis I examine the phenomenon of Paleolithic cave art and what it might be able to tell us about the minds of the Cro-Magnon artists who produced it. I survey the paintings that have so far been discovered, as well as the processes involved in creating them. I also discuss and critique a selection of the many theories that have attempted to explain the motivation behind this radically different type of human behaviour. But due to the lack of hard evidence, none of these theories are ever likely to be fully substantiated. So a more promising line of investigation I take is to appraise the cognitive abilities Cro-Magnons would have needed to produce the paintings - and this then allows me to consider whether cave art was indicating any new cognitive development. I therefore highlight one of the effects that creating cave paintings had: it allowed information from the brain to be stored in the environment. But the manner in which this form of epistemic engineering might enhance human cognition is a hotly debated subject. I examine two theories: the extended mind hypothesis, and the theory of niche construction. In concluding this thesis, I argue that cave art seems more like an example of epistemic niche construction than a constituent of an extended mind. 


\section{Acknowledgements}

First of all I have to thank my primary supervisor Professor Kim Sterelny. Kimbo, thanks for your undying patience with both me and this project, along with its many previous incarnations. I am sure, like me, you wondered at times whether this was ever going to be completed, but we both hung in there and I got it done.

Nick Agar was my second supervisor, and although I never ultimately made use of his assistance, he was the one who first introduced me to consciousness studies. It was to be the start of a long and winding path that ended up in the caves of Western Europe!

My initial contact with Victoria's philosophy department was swift: I arrived on campus for an informal chat following a brief and speculative phone call made a mere thirty minutes earlier! The first person I met was Ed Mares. I wandered into an office that looked like a bomb had hit it, and as I introduced myself I suddenly realised that all I really wanted to do right there and then was study philosophy - even though I had never formally encountered the subject before. Fortunately, Ed was so helpful, and in less than another thirty minutes he had provided me with a route into the department and a plan that would allow me to study for this Masters Degree.

There are so many people to thank in Victoria's philosophy department, but I shall only mention a few by name. Tony Scott has been such a great help: whenever I had a question, often a stupid one, Tony would always listen and give me an answer that would make me realise that some of this stuff is actually pretty hard to fathom; he also spent much time reading through this thesis and providing me with excellent feedback, and at the level that I needed. Matt Gers provided me with the article that linked cave art and the extended mind - just at the time when I needed some impetus. Dan Turton (sorry, but Dan Weijers just doesn't sound right!) was always around for a chat and willing to impart some wise words and guidance. Whilst Ben Jeffares provided plenty of technical advice when I got totally bogged down. The department even managed to supply me with a few extra golfing buddies!

Finally, my thanks go to my partner Carole, who did her best to remove many of my day-to-day distractions, which allowed me to concentrate on completing this thesis. 


\section{Contents}

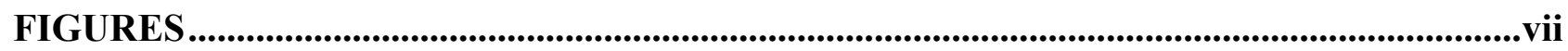

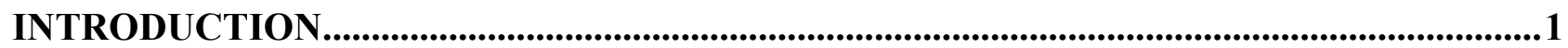

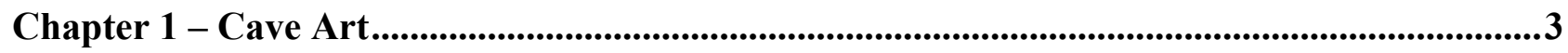

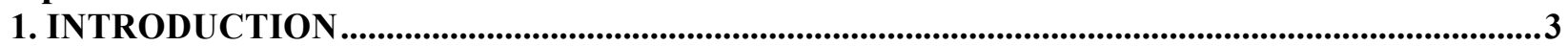

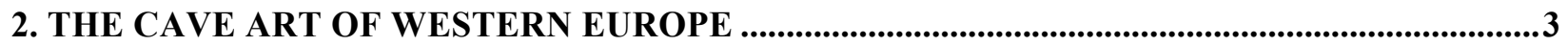

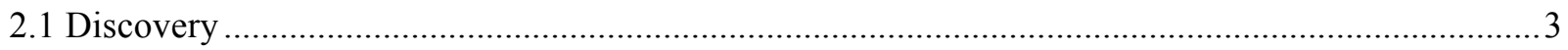

2.2 Geography

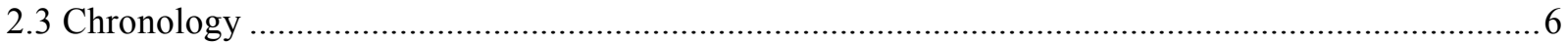

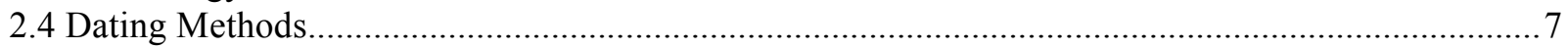

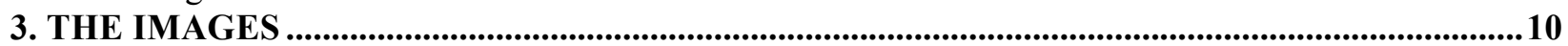

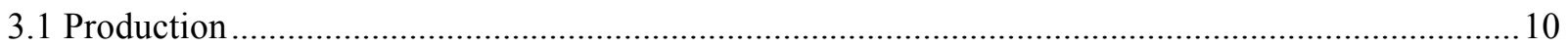

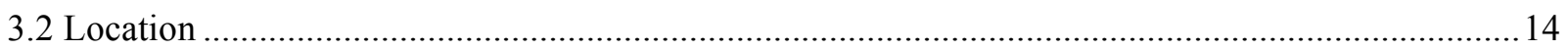

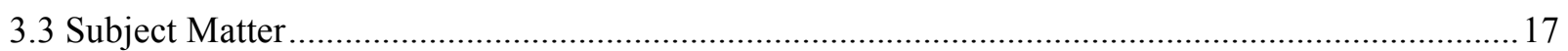

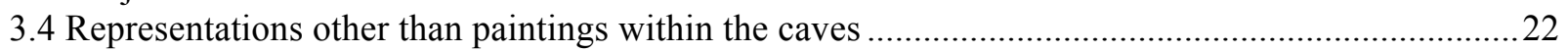

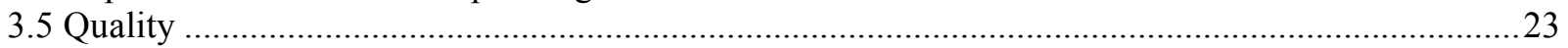

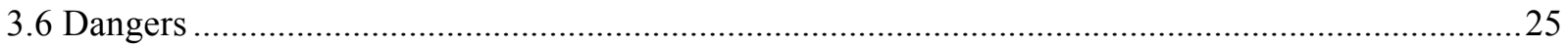

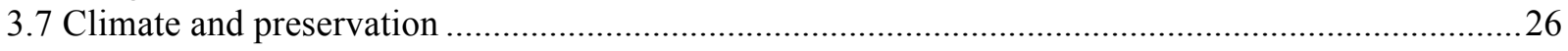

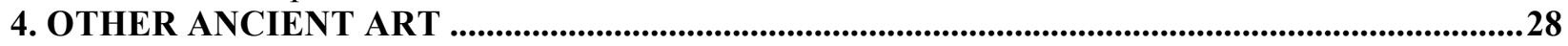

4.1 Other Paleolithic images and representations found in Western Europe........................................28

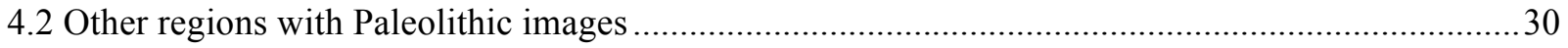

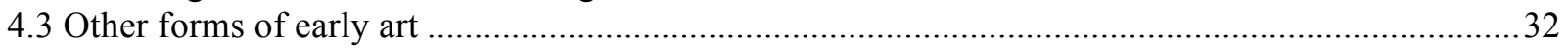

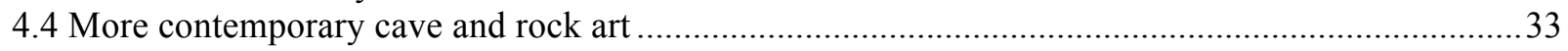

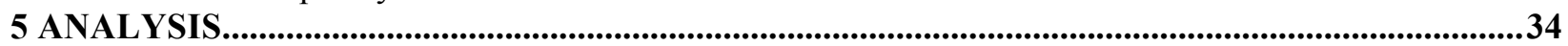

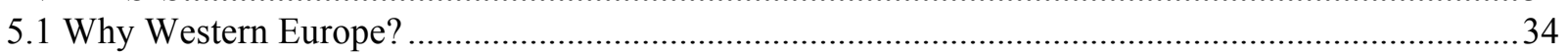

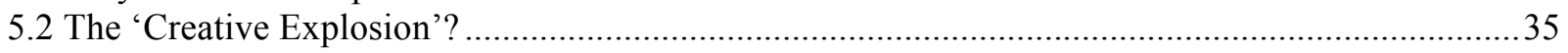

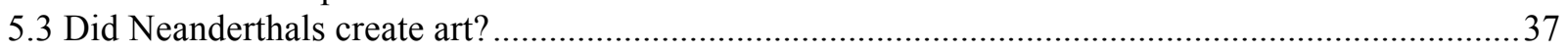

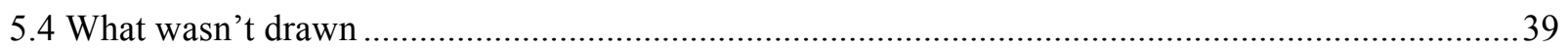

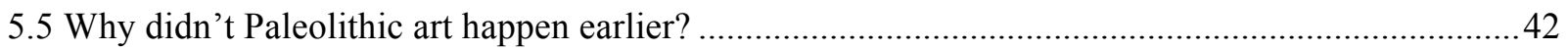

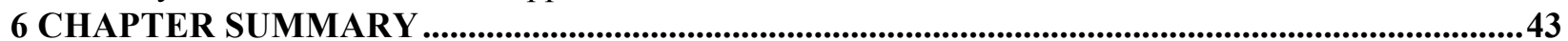

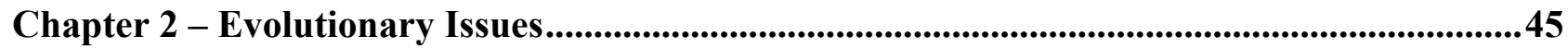

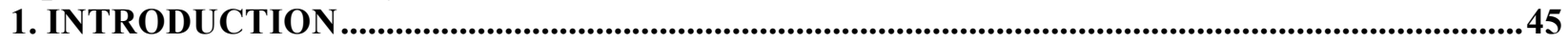

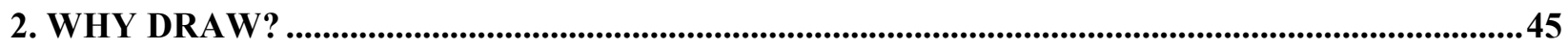

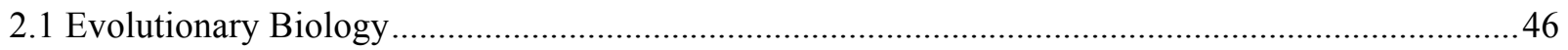

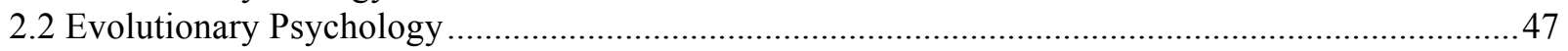

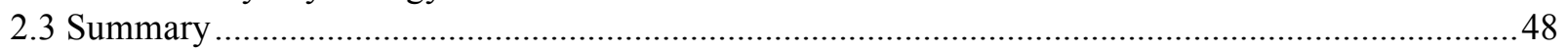

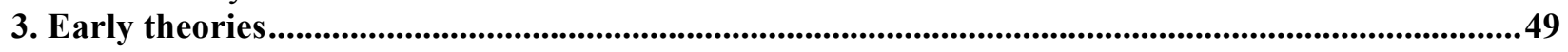

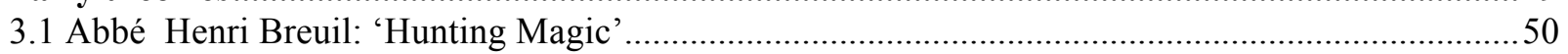

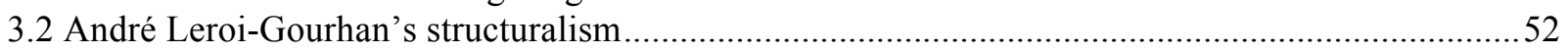

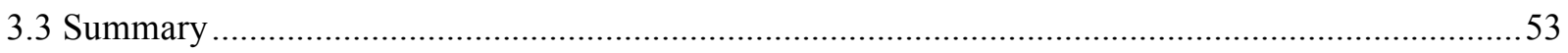

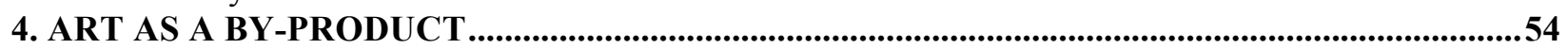

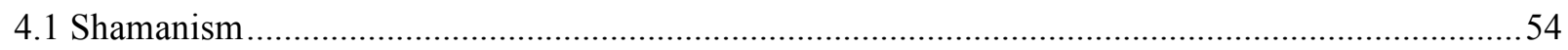

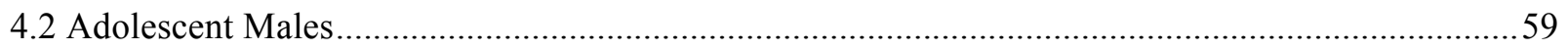

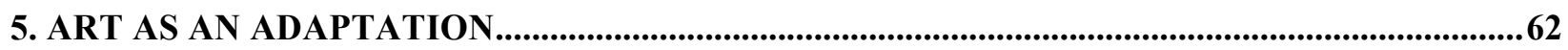

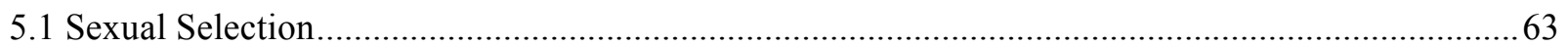

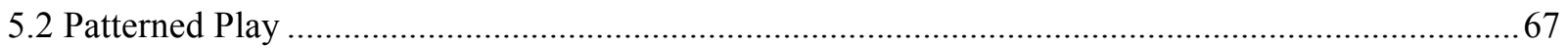

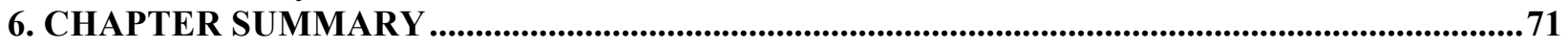

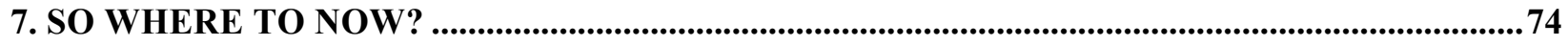


Chapter 3 - The Cognitive requirements of Cave Art ........................................................................75

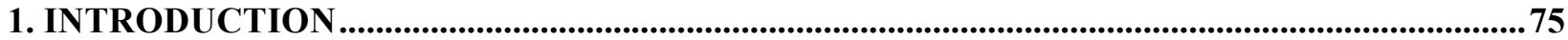

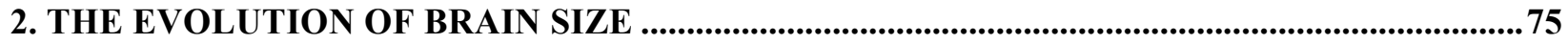

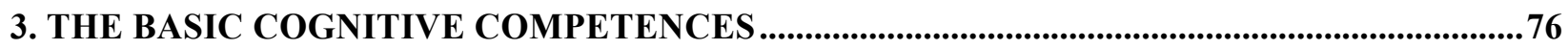

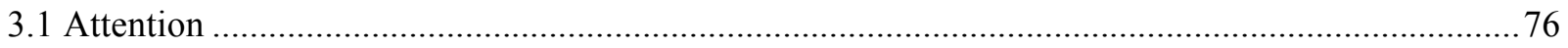

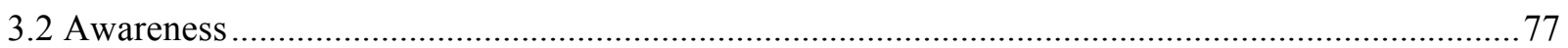

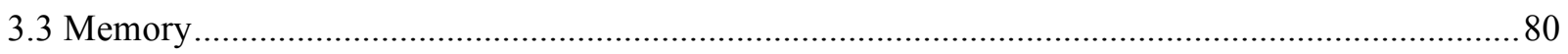

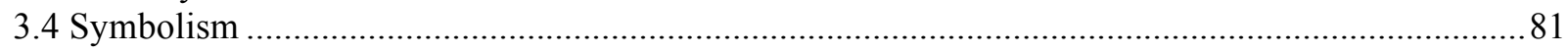

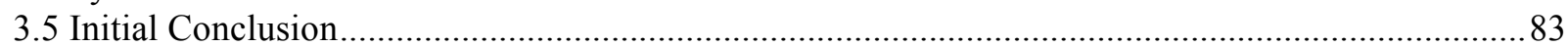

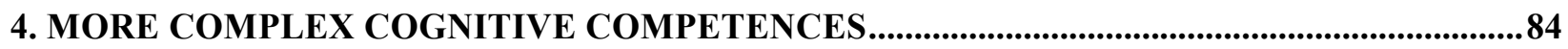

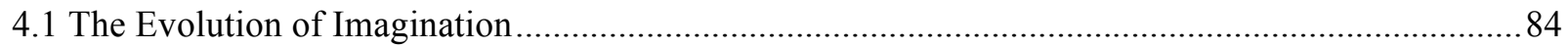

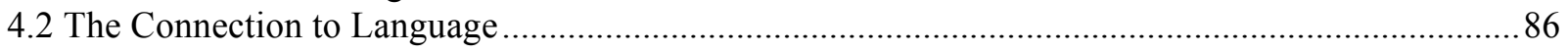

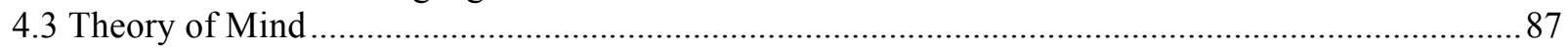

5. CHAPTER SUMMARY ...................................................................................................................91

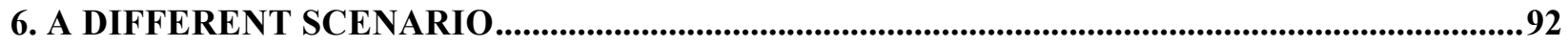

Chapter 4 - Cave Art: Freezing a Thought? ...........................................................................................93

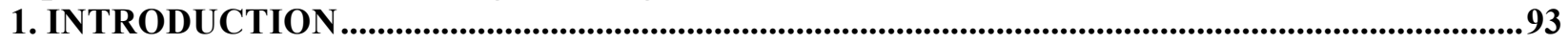

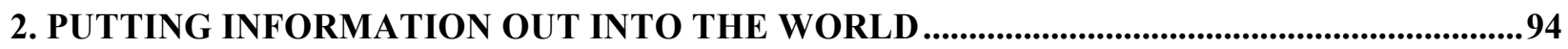

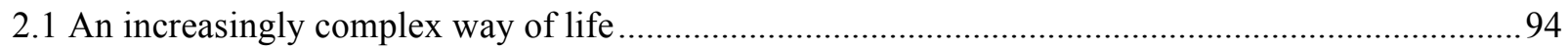

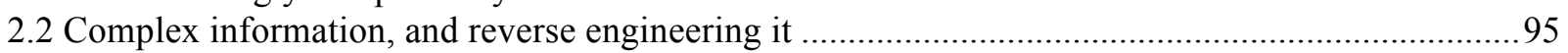

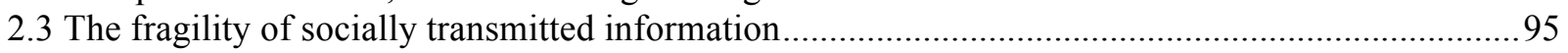

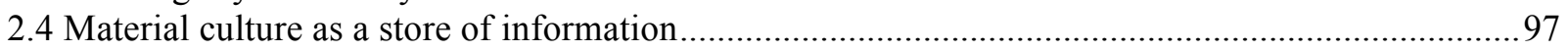

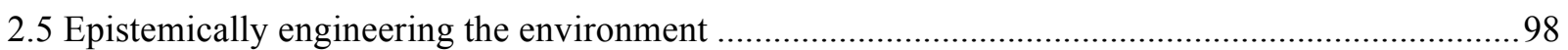

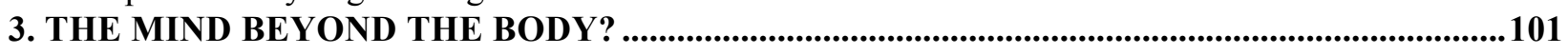

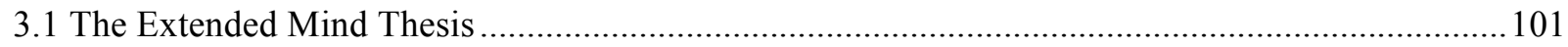

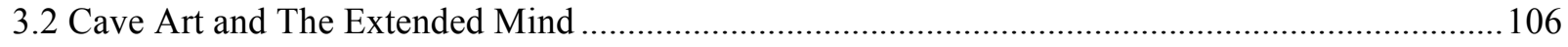

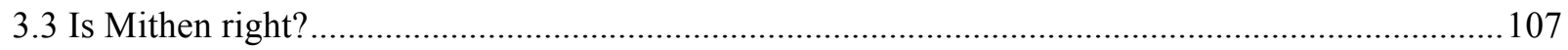

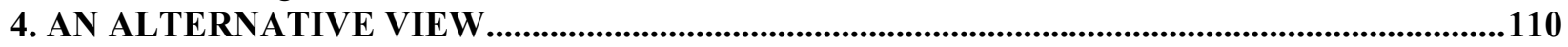

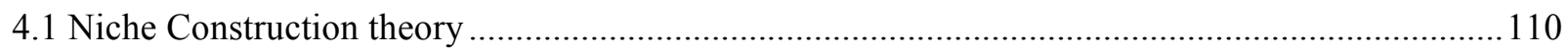

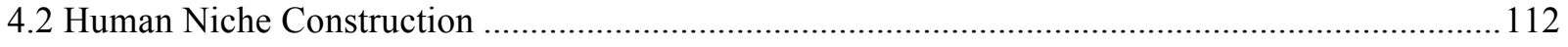

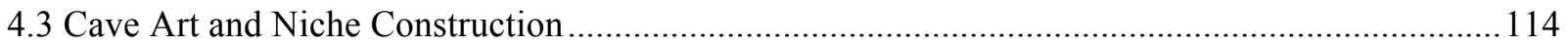

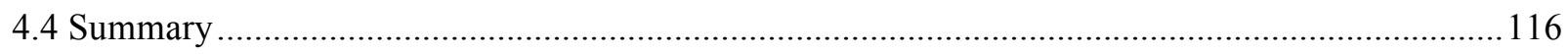

5. CHAPTER SUMMARY ….............................................................................................................117

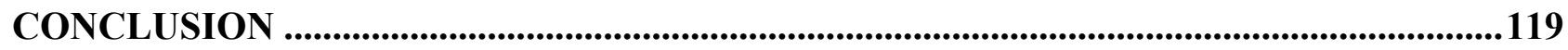

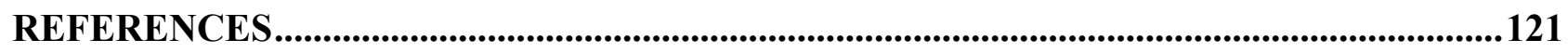




\section{FIGURES}

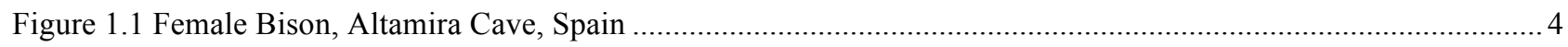

Figure 1.2 Some of the main sites of Paleolithic art in Western Europe ................................................................... 5

Figure 1.3 Chronological table of cultures and periods of the Upper Paleolithic in which cave art flourished ............... 7

Figure 1.4 Jean-Marie Chauvet, standing next to the Panel of the Horses, Chauvet Cave, France ................................ 9

Figure 1.5 Representation of a Bison or Mammoth using a stippling effect, Chauvet Cave, France............................ 14

Figure 1.6 Black dappled horses drawn around a crack in the wall, Pech-Merle Cave, France................................. 15

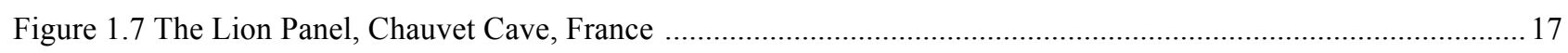

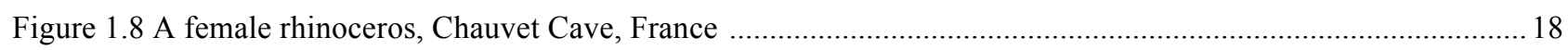

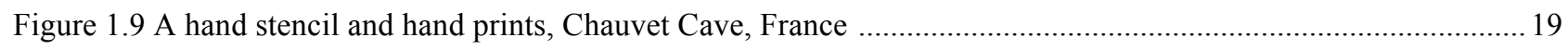

Figure 1.10 Pubic triangle drawn on a stalactite-like protrusion, Chauvet Cave, France ............................................20

Figure 1.11 'Venus with horn' bas-relief, Laussel Shelter, France ….................................................................20

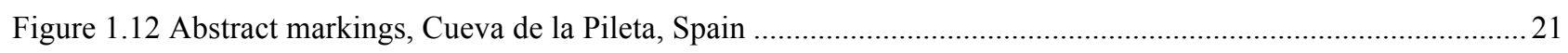

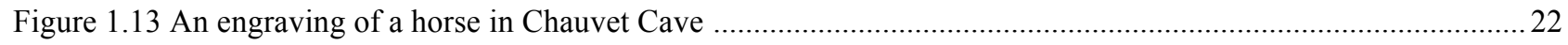

Figure 1.14 Bison moulded from clay, Le Tuc d'Audoubert Cave, Ariège, France ...............................................23

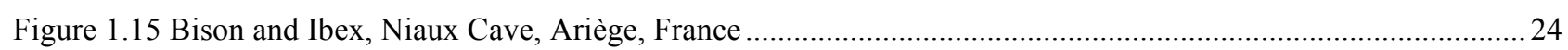

Figure 1.16 Decorated spear thrower made from reindeer antler, Ariège, France …..............................................29

Figure 1.17 Venus of Hohle Fels figurine made from mammoth-ivory, southwestern Germany ..................................29

Figure 1.18 'Vogelherd horse', made from mammoth ivory, Cave of Vogelherd, Germany ...........................................30

Figure 1.19 'Lion man’ figure made from mammoth ivory, Stadel Cave, Hohlenstein, Germany …...........................30

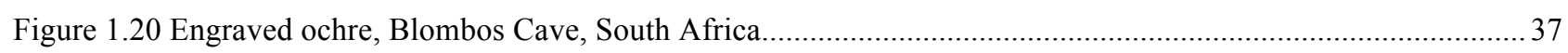

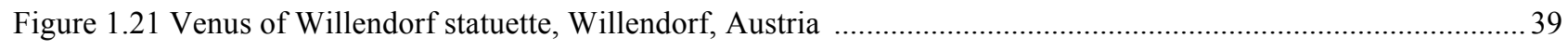

Figure 1.22 Composite being 'The Sorcerer', Cave of the Trois-Frères, France ........................................................ 41

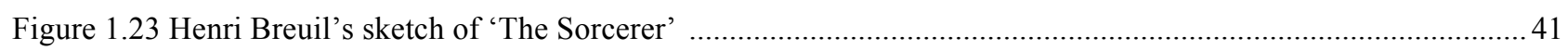

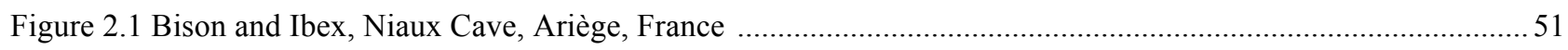

Figure 2.2 San rock painting of hybrid human-animal figures and an eland ...........................................................56

Figure 2.3 A cave bear skull placed on a fallen rock in Chauvet cave .......................................................................58

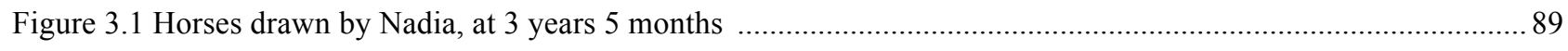

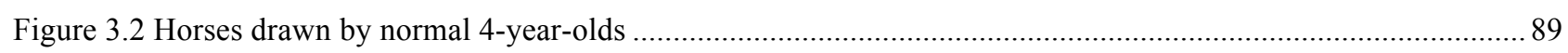

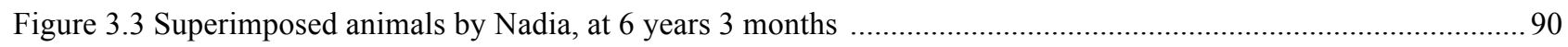

Figure 3.4 Composite animal, part giraffe, part donkey, by Nadia at approximately 6 years .................................... 90

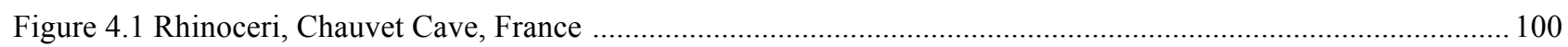




\section{INTRODUCTION}

Cave art appeared in Western Europe some 40,000 years ago and perhaps even earlier in other parts of the world, but what function - if any - did it play in human cognitive evolution? The creation of a cave painting involved a considerable cost to the individual in terms of the amount of time and energy spent collecting all the necessary materials. Pigments and sources of lighting all required a lot of effort in both their gathering and subsequent preparation. Furthermore, paintings made deep in caves also involved an element of risk with a need to negotiate hazardous routes through the cave systems. These combined costs would seem to indicate that the motivation to produce cave art was strong.

The production of cave art in Western Europe appears to herald an important advance in the behaviour of early humans, and this is what I intend to examine in this thesis. It is my opinion that the creation of cave art by our ancestors was indeed a truly significant cognitive event. It demonstrated an ability to place raw information in the form of naturalistic representations out in the world, which was an unparalleled cognitive feat at that particular time in our evolution. Furthermore, cave art clearly demonstrates an indubitable awareness of their awareness of the environment - something that other cognitive 'tricks' do not necessarily require.

But what sort of cognitive change does cave art indicate? Before being able to address this question, and demonstrating that cave art was a major development in our cognitive evolution, I will first have to consider the skills and demands, both physical and psychological, that it required. In chapter $1 \mathrm{I}$ therefore begin with a review of the phenomenon of cave art and focus on its discovery in Western Europe, as well as investigate its history and where it has been found. I also highlight the tools and materials that were used. Following this, I then analyse the images: what did they draw, and where in the caves did they choose to place them. I conclude the chapter by reviewing what other art existed in the world at around this time, and then also attempt to address some of the initial questions that cave art's discovery has evoked.

Once an overview of cave art has been provided, in chapter 2 I discuss the reasons why it might have been drawn. There are two types of question here. The first question asks what the motivations were for the artists' to make cave paintings. Whilst a second question goes much deeper, and requires an evolutionary explanation: was the act of producing cave art an evolutionary adaptation 
that in some way improved our ancestors' chances of survival? Or was it a by-product of our evolutionary history that provided no selective advantage. When analysing behaviour from an evolutionary viewpoint, the disciplines of evolutionary biology and evolutionary psychology are important, so I begin the chapter with a brief summary of them both. I next move on to summarise some of the major early theories put forward to explain why cave art occurred. These early theories have now mostly been dismissed, but they do provide a background to the more popular modern theories that I subsequently discuss. I finish the chapter by evaluating all the theories that I have discussed, and then consider how else cave art can be analysed.

In chapter 3 I move away from conjecture and instead focus upon what we can deduce from cave art with near certainty. I therefore examine what cognitive skills would have been required to create the paintings. The production of a cave painting is an incredibly complex cognitive process and an artist needed to employ many different skills and competences. After an initial review of human brain evolution, I examine the basic cognitive competences that would have been called upon. For example, the drawing of a painting requires a good deal of concentration, and remaining focussed on the task at hand, whilst resisting distraction, is a great cognitive skill. When combined, these basic brain functions produce much more complex cognitive abilities; I therefore appraise some of these competences and consider how they might connect to what was cognitively required for cave painting. I conclude by addressing the question of whether cave art could be indicating any other cognitive development.

So, having analysed in the previous chapter what cognitive skills our ancestors' brains were capable of, I next move on in chapter 4 to evaluate what other cognitive developments might have been happening. Organisms often manipulate their environments to improve their chances of both survival and reproduction, but some also place information there too. Humans manipulate their environments as well, but they have also slowly evolved to use it as a store of information far better than any other organism. Cave art appears to be a very early example of the epistemic engineering of the environment by humans, but how can we best interpret this behaviour? Some philosophers, such as Andy Clark and David Chalmers, believe that this epistemic engineering is best explained by their theory of an extended mind. Steven Mithen has argued that cave art represents an early stage in extended mind's development. I investigate these claims, before considering an alternative method of analysing the modification of an organism's environment: the theory of niche construction. I conclude by explaining why I believe that cave art is more likely to be an example of epistemic niche construction. 


\section{Chapter 1: Cave Art}

\section{INTRODUCTION}

The beautiful Paleolithic cave paintings of Western Europe were a remarkable achievement by an apparently primitive people. The naturalistic style and accuracy of the paintings was so advanced that it was far ahead of its time, with many of the styles - such as the ability to show movement only rediscovered many thousands of years later. To create these paintings, in such inhospitable locations, required many skills and lots of planning, all of which indicates that the people of the Upper Paleolithic were much more advanced than many had believed.

In this chapter I will begin by providing an overview of the Paleolithic cave art of Western Europe, including details of its initial discovery, where it has been found, and how old it is. In the next section I will examine in more detail the images themselves: how they were made, where in the caves they were located, and what was represented, as well as other factors such as conservation. I will then try to place cave art in the context of other ancient art found throughout the world, and attempt an initial assessment of its significance.

\section{THE CAVE ART OF WESTERN EUROPE}

\subsection{Discovery}

The phenomenon of cave art is a comparatively recent revelation and it has only been just over a century since our first knowledge of it came to light. In 1879, on the Cantabrian coast of Northern Spain, Marcelino Sanz de Sautuola, a local landowner and amateur archaeologist, made the first ever discovery of prehistoric cave paintings, which he claimed were from the Paleolithic era. Whilst performing some investigative research on the caves near to where he lived, and accompanied by his young daughter Maria, he came across one particular cave and ventured inside. As he was looking down at the ground searching for fossils, Maria raised her light, looked upwards, and saw the now famous paintings on the ceiling (Spivey 2005). Unfortunately, due to the superb quality of the paintings they were declared fake and the aspiring archaeologist was ridiculed for attempting to 
break into the established archaeological circles of the day. He died in 1888, and it wasn't until 1902 that these cave paintings were finally recognised as authentic (Clottes and Lewis-Williams 1998). The many paintings in the Cave of Altamira have been dated from 13,500-19,000 years ago (Clottes 2008).

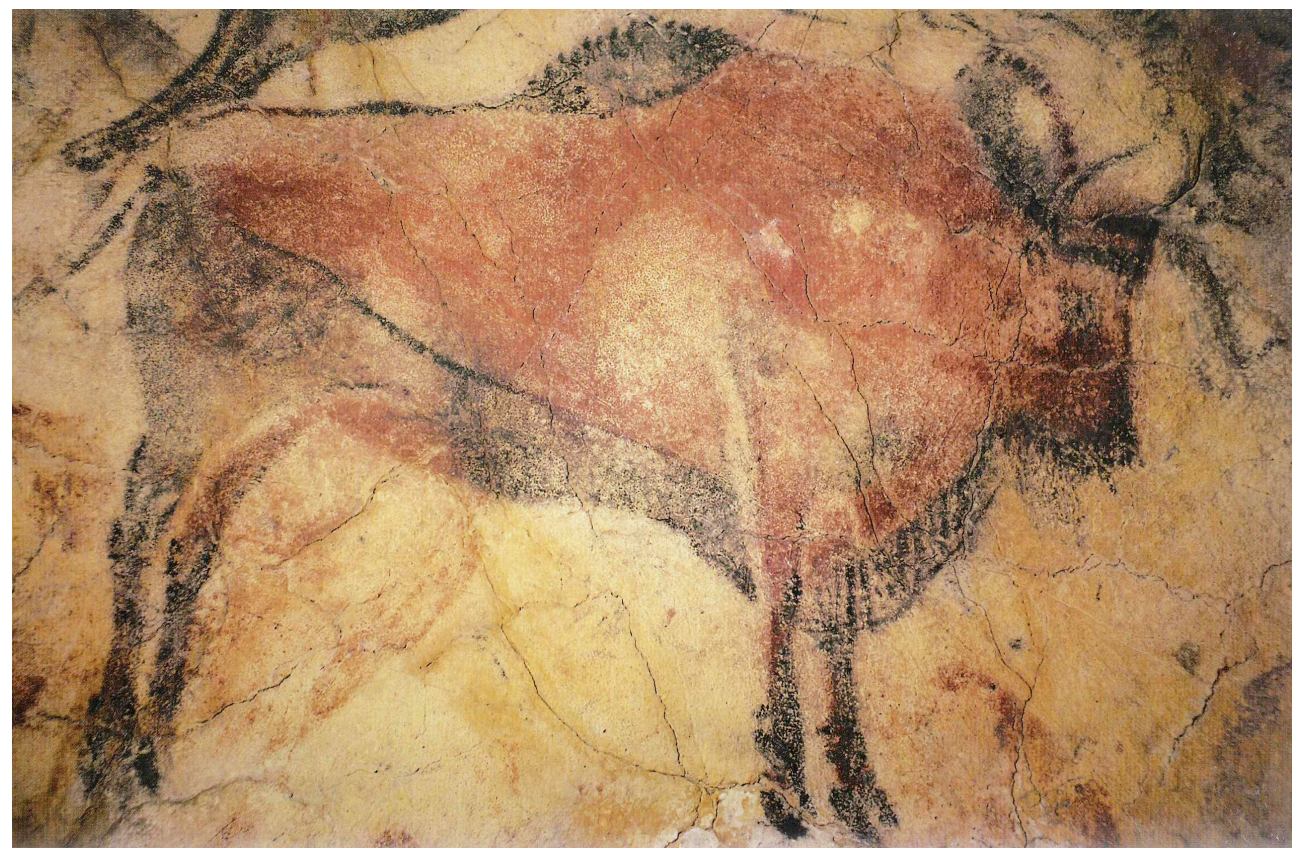

Figure 1.1 Female Bison, Altamira Cave, Spain - approximately 15,000 yrs old (Early or Middle Magdalenian) (Clottes 2008)

Major finds of caves containing Paleolithic art in the form of paintings and engravings are still continuing. In recent times there have been two major discoveries in the south of France: Chauvet and Cosquer caves. Chauvet Cave (see below for more details) was only found in 1994 and what it contained has led to a radical re-evaluation of previously held assumptions. The discovery of Cosquer Cave in 1985 (but only made public in 1991) is even more surprising, because its entrance was located 37 metres below sea level. From the cave's entrance a narrow access passage of some 175 metres had to be carefully negotiated by divers over several years of expeditions before its treasure was finally revealed. And the only known cave art (engravings) in the United Kingdom at Creswell Crags in Derbyshire was discovered as recently as 2003 - yet the area had been regularly frequented since the Victorian era; it is currently the most Northerly example in Europe. These recent discoveries make it seem highly likely that many more important archaeological finds pertaining to cave art will be made.

In contrast to the art found in caves, our knowledge of rock art, which is found out in the open on exposed rock, has been written about and recorded by various explorers in different parts of the 
world for many hundreds of years - and even for several thousand years in the case of some Chinese writings (Bahn 1998). But the rock art found so far is neither as old nor as exquisite as the cave art that has been discovered in Western Europe.

\subsection{Geography}

The earliest known examples of cave art have predominantly been found in Western Europe, with the main archaeological finds being in south-west France and north-east Spain, although examples within Europe have been found as far away as the Ural mountains of Western Russia. France and Spain account for around $95 \%$ of the cave art that has been found so far (Clottes and LewisWilliams 1998; Clottes 2008). To date there have been approximately 350 caves discovered in Western Europe, together containing thousands of Paleolithic images (Guthrie 2005).

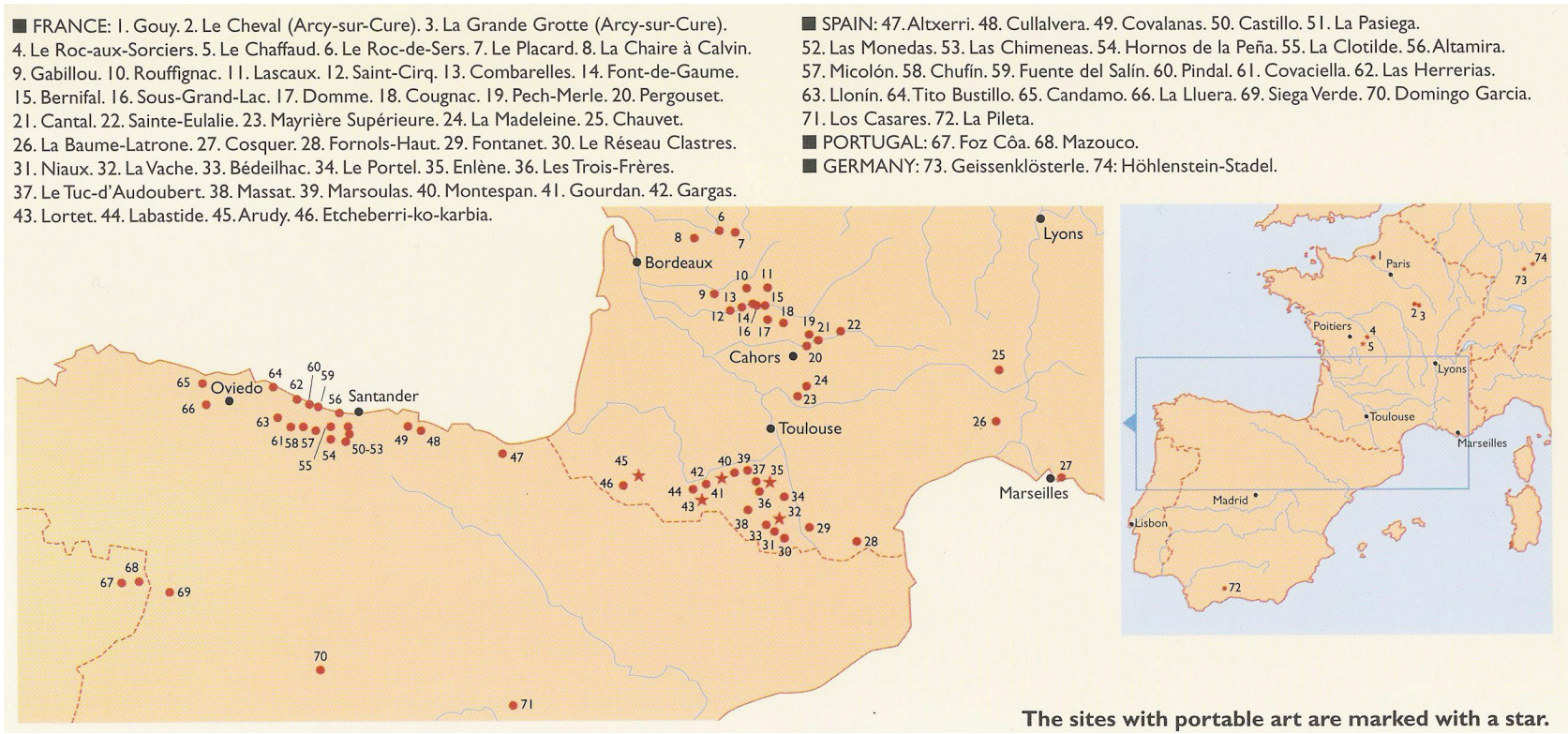

Figure 1.2 Some of the main sites of Paleolithic art in Western Europe (Clottes and Lewis-Williams 1998)

The discovery of a decorated cave is often made by chance, but a hint that one may exist nearby can be provided if portable art from the Upper Paleolithic, such as engraved bone carvings, has been found in the same area. If the inhabitants were capable of creating portable art then they were probably also capable of creating parietal ${ }^{1}$ images. Indeed, Paul Bahn states that this was how Creswell Crags in England was discovered in 2003; a group of cave art experts had noted that whilst the area contained caves that were known to have been occupied during the Paleolithic, and

\footnotetext{
1 Artwork created on cave walls or large blocks of stone.
} 
where many artefacts had been discovered including a bone that had a horse's head engraved on it, no examples of cave art had previously been found there (Pettitt, Bahn et al. 2007). A detailed examination of the area eventually found the faintest remnants of parietal images within the caves.

\subsection{Chronology}

According to current archaeological data, anatomically modern humans (Homo sapiens) arrived in Western Europe approximately 40,000 years ago (Clottes 2008). Prior to the appearance of this second species of the Homo genus, the area had been occupied by Neanderthals (Homo neanderthalensis) alone for some 200,000 years or more. Anatomically modern humans had migrated from Africa after a long journey lasting many millennia that took them through the Middle East and then Eastern Europe, before eventually settling in the west of Europe. They have been termed Cro-Magnons after the cave in southwest France where their remains were first found. Unlike the Neanderthals who were from a separate evolutionary line, anatomically modern humans were our direct ancestors. They were very much like us physically and in brain size.

It has been somewhat controversially claimed that the arrival of anatomically modern humans in Europe coincided with a major change. In this period our ancestors in the region began to adopt modern-day behaviours that set them apart from their earlier generations; hunting techniques improved and tool manufacture became much more complex. This significant change in behaviour has been referred to as 'The Creative Explosion' - a phrase coined by John Pfeiffer and used as the title of his 1982 book on the subject. The period in time that these changes occurred is classified as the Upper Paleolithic (but also known as the Late Stone Age) and it lasted from approximately 40,000 years ago until 10,000 years ago. But the evidence supporting the claims of there being a creative explosion has been called into question, as I will discuss later on in this chapter. It was also during this period that the production of parietal art (on walls, and in the form of cave paintings and engravings) and portable art (in the form of easily transported figurines and carvings) first appeared.

At present the oldest known cave art, discovered at Chauvet Cave, has been dated to approximately 32,500 years ago (Chauvet, Brunel Deschamps et al. 1996). The table below provides a summary of the approximate age of some of the more important sites containing cave art, along with the respective cultural era that it is from. 


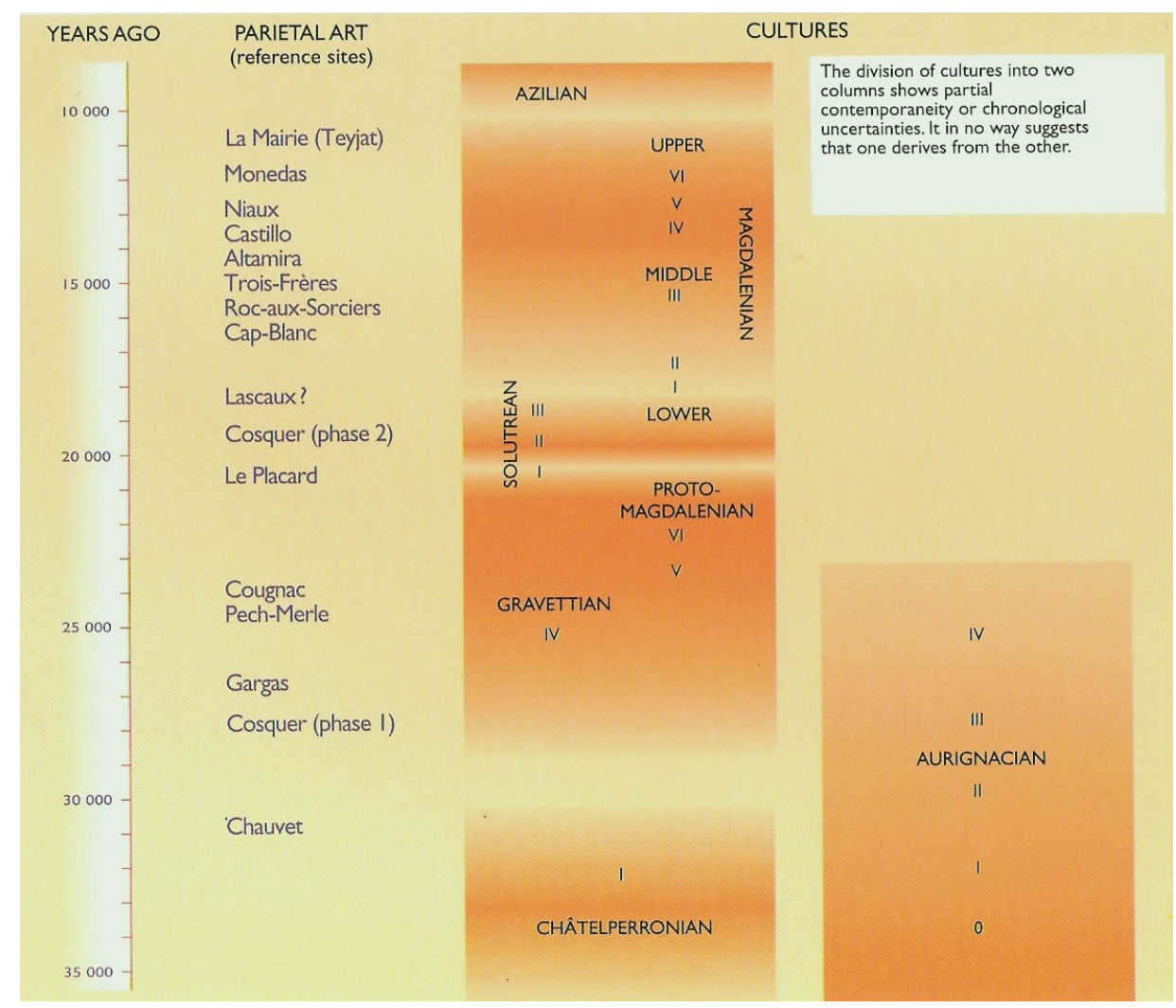

Figure 1.3 Chronological table of cultures and periods of the Upper Paleolithic in which cave art flourished (Clottes and Lewis-Williams 1998)

\subsection{Dating Methods}

The dating of inorganic materials such as pigment can be problematic and until relatively recently the assessing of a cave painting's age has been very difficult. Other archaeological discoveries of inorganic objects such as handaxes and portable carvings can be subjected to contextual dating methods because they accrue layers of earth and other deposits on top and below themselves, but cave paintings do not. These layers provide extremely useful and reasonably reliable information for evaluating how old an item may be. So cave paintings have no archaeological context in which they fit. In contrast, objects lying on the ground have several advantages when it comes to dating. For example, other items associated with a particular era may surround them, changes in climate may produce different effects indicating a specific date, or organic materials found nearby can be radiocarbon dated and the object dated by association. Pollen analysis is an additional method available to assist in the contextual dating of archaeological finds by calculating the age of pollen found in the habitation layers that surround them (Ruspoli 1987).

One method for dating a cave painting is to examine the subject matter: many animals such as woolly mammoths, cave bears, and certain types of deer have approximate extinction periods and 
thus any cave paintings of them would specify a period after which the painting could not have been drawn. Dating can be further complicated because some paintings were made in several stages, sometimes separated by many thousands of years, whilst others were painted over and covered by newer images (Clottes and Lewis-Williams 1998). So whilst dating techniques are reliable, their inability to be used effectively on some cave paintings has resulted in age dispute claims.

Current radiocarbon dating methods can be applied to paintings that were drawn using charcoal, as this is an organic material, but any paintings made using inorganic pigments cannot be dated using this technique. Luckily, sometimes paintings were first sketched out in charcoal before paint was applied over the top of them and thus the charcoal part can be dated (Ruspoli 1987; Bahn 1998). Furthermore, if pieces of organic matter such as strands of fibre from the brushes, or splinters of wood used in applying the colour are found in the painting's pigments, then these too can be accurately dated using the radiocarbon dating method (Bahn 1998). One additional indication of the date of a cave painting - or at least when the cave has been used - is the carbon deposits that were often left on the walls by the different methods of lighting used. The paintings in Chauvet Cave were doubted for some time until an analysis of torch marks left on the calcite that had accumulated over the top of a painting was dated and confirmed the cave's authenticity (Chauvet, Brunel Deschamps et al. 1996).

More recently it has been discovered that some cave painting pigments consist of a mixture of powdered ochre and an organic binding agent such as urine, saliva or animal blood. New techniques, such as Accelerator Mass Spectrometry, are able to separate out even the smallest traces of these organic constituents that can then be dated. The introduction of AMS (a supplement to radiocarbon dating) has meant that now only a very small amount of organic matter (such as charcoal) is needed for accurate dating - prior to its use, a large sample was needed, so much that the amount required would have destroyed the image (Chauvet, Brunel Deschamps et al. 1996). Additionally, X-ray diffraction and Infrared Spectrometry are amongst a number of methods that can identify the mineral constituents of the pigments used, which can then assist in the dating of each painting.

Improved radiocarbon dating methods are now able to accurately date cave paintings to within plus or minus several hundred years. The technique can be used to date objects up to 60,000 years old and does not provide an exact age but instead produces a date range with an accompanying degree of accuracy. So for instance, a painting in Chauvet Cave that has been dated as being 32,410 +/- 
720 years old, actually has a $68 \%$ chance of being accurate; for $95 \%$ accuracy the amount of variation has to be doubled, i.e. 32,410 +/- 1440 (Chauvet, Brunel Deschamps et al. 1996).

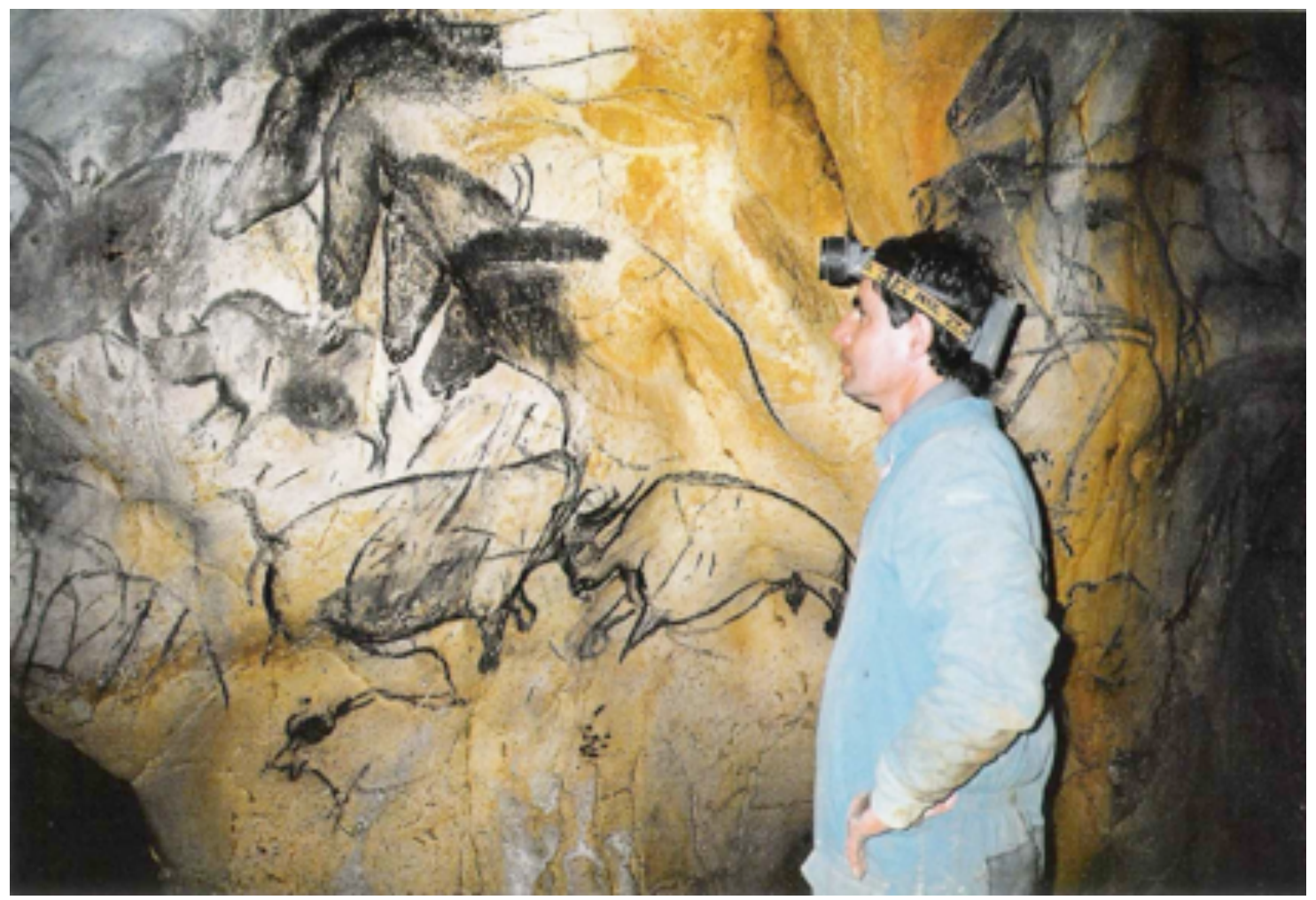

Figure 1.4 Jean-Marie Chauvet, one of the discoverers of Chauvet Cave, standing next to the Panel of the Horses which has been dated up to approximately 32,000 years ago (Chauvet, Brunel Deschamps et al. 1996)

The increased accuracy of dating methods has helped to verify the disputed authenticity of caves such as at Cosquer in France and it has also been able to detect fakes such as the cave at Zubialde in Spain (Bahn 1998). In 2008, the paintings from the Cave of Altamira were re-dated using the new 'uranium-series dating' technique and the oldest have now been shown to be between 25,000 and 35,000 years old (Pike 2008). This method involves analysing the carbonate deposits that slowly form over the top of the paintings.

But the ability to accurately date cave paintings is not only important for verifying authenticity. Accurate dating also allows cave paintings to be placed chronologically alongside other aspects of material culture and social life of the Upper Paleolithic. And as a consequence, this type of behaviour can then be compared and contrasted with other behaviours of that era, allowing philosophers and scientists the possibility of analysing its relative significance. 


\section{THE IMAGES}

Producing the images found in the caves of Western Europe required a great deal of planning and effort. Firstly, the materials needed for their creation had to be assembled; then a cave's terrain had to be negotiated; and finally, a decision had to be made for what image to draw and where to place it. In this section I will discuss the practicalities of creating cave art, along with the chances of it being preserved until the present day.

\subsection{Production}

The materials and methods used to produce cave paintings were varied and the artists used a range of colours to create naturalistic representations. The easiest and most accessible material for marking a cave wall was to use the charcoal from the remains of fires and a large percentage of the cave art produced was drawn this way. As previously stated, even when pigments were used, outlines of the intended paintings were sometimes first drawn in charcoal.

\subsubsection{Colours}

Of the pigments used for painting, ochre was a popular choice as this substance has a long history of being utilized by our ancestors - possibly for body art, but it has many uses including medicinal qualities that make it suitable for dressing wounds (Bahn 1998). Ochre was also a component of glues made for producing compound tools (Wadley 2010). Possible evidence of ochre use dates back as far as 800-900,000 years ago in South Africa (Bahn 1998). Ochre dated to 200-300,000 years ago has been found in India with marks on it that appear to indicate that it had been deliberately rubbed against some rock (Bahn 1998). Although interpretations such as this are possible, in many cases there is an equally plausible explanation that the marks were made by natural geological processes. There have been discoveries of ochre dating from 250-300,000 years ago at a rock shelter in Bečov in the Czech Republic and at Terra Amata in France. In both cases the ochre was found in non-naturally occurring locations that had been occupied by early human ancestors and bore surface scratches that were deliberately made (Bahn 1998). The combined factors of where it was found, along with the accompanying surface markings, support the claim that these natural pigments were actively sought out. A more contemporary finding of red ochre, thought to be used as part of a burial ritual 100,000 years ago, has been discovered at Qafzeh Cave in Israel (Hovers, Ilani et al. 2003). 
Manganese and iron oxides were also used as pigments, to produce black and red respectively. It is interesting to note that almost all cave paintings were drawn in these two colours - even though the artists also had access to the colours of yellow and white, these were rarely used (Clottes and Lewis-Williams 1998). It also appears that the artists deliberately changed the colours of these raw pigments by heating them, based upon pieces of ochre found in a hearth by André Leroi-Gourhan that were at various stages of oxidation (Ruspoli 1987). Tools have been found at several sites in South Africa dated from 164,000 years ago that support this sophisticated use of fire, because they show signs that heat treatment was used in their engineering (Brown, Marean et al. 2009).

Often the pigments were extracted from their original source and then moulded into crayons; to do this, the raw material was broken down into a powdery form and then washed so that any impurities could be removed. Finally, a binding agent was added to allow the powder to stick together and although organic materials such as blood or urine were sometimes used, experiments at Lascaux found that the best binding agent was actually the water from the caves as it is rich in salts that would have helped to form a hard end product (Ruspoli 1987). Crayons - like charcoal - could be applied directly to the walls, but raw pigments had the disadvantage of needing to be first mixed with an applicator before they could be painted on a cave wall; there were various options for doing this and turning it into liquid form by adding the binding agents mentioned above was the easiest option. Artists could use fingers or hands to smear the liquid onto the wall, but the use of twigs was another simple way to mark the walls with the pigment. Alternatively, they also made brushes from hair and bristle, or used pieces of animal fur as a kind of sponge (Ruspoli 1987). A further method they used was to spray the pigment from their mouths after chewing it ${ }^{2}$ (Guthrie 2005).

It has been reported that many of the older pieces of ochre contain smooth surfaces, something that could have resulted from rubbing it against human skin (Bahn 1998). So if ochre had been used for decorative purposes such as body painting long before the advent of cave art, then this may to some extent explain why pigments were used even when charcoal was plentiful, as it was already available due to another use. And as previously mentioned, it may have originally been used for medicinal purposes before then.

\footnotetext{
2 For an interesting explanation of this method see page 126 of Bahn, P. G. (1998). The Cambridge illustrated history of prehistoric art. Cambridge, U.K., New York : Cambridge University Press.
} 
The acquisition and subsequent processing of the pigments used to produce the paints for cave paintings involved a significant cost with respect to both time and resources. The mining, transfer, and refining of these natural pigments would have been a major task, not simply a minor distraction from their daily life-sustaining activities. Furthermore, the pigments were often sourced from locations many miles from where the caves were situated (Ruspoli 1987). Overall then, the amount of preparation involved in manufacturing the pigments needed for making the paintings was substantial; it was not simply a case of picking up a coloured piece of stone and drawing with it.

\subsubsection{Lighting}

In the process of creating cave paintings, the artists would have spent many hours or even days inside the deeper caves searching for appropriate locations to place their drawings - and a prerequisite of being able to do this is that they needed some form of lighting. The easiest form of lighting was to use a piece of burning wood, a torch. As the torches burnt down they were often wiped against the walls to remove the excess charcoal (Chauvet, Brunel Deschamps et al. 1996) these telltale marks have been found in many caves and have been used in radiocarbon dating. But the problem with torches is that they did not last too long, so the main light source were lamps that used a fuel of animal fat or tallow - which were also valuable food resources - and a wick often made of lichen, juniper, or grass. The fuel could either be cut directly from the animal (e.g. the renal cavity of an ox), or sourced from bone marrow (Ruspoli 1987). The lamps were made out of pieces of stone with a handle at one end and a wider, ground out area, at the other end. Well over a hundred of these lamps have been found in the Cave of Lascaux, many on the ground directly below the paintings (Ruspoli 1987). These Paleolithic lamps were not very bright; they were much dimmer than the light emitted by a candle - but human eyes can adjust relatively quickly to poor quality light and so it would have proved adequate for their needs.

The torches and lamps would also have produced important and provocative effects: the weak and flickering light would have made the contours of the cave appear to come alive with fleeting shadows appearing everywhere. Indeed, many of the cave paintings are best seen in this sort of lighting - and seeing them in this way would give a far better idea of what these ancient people saw as they ventured into the caves. Modern lighting produces completely different effects and the colours in the paintings that we now see would not have been anything like as vivid in the much dimmer light. Soot and smoke deposits from these lamps and torches have been found on top of the paintings, and these remnants have sometimes been used to authenticate the age of these paintings (Guthrie 2005). 
Once again, like the collection and processing of pigments, the use of fat-burning lamps involved appreciable costs. Artists first had to create the stone lamps by grinding out and shaping the bare pieces of rock; they then had to acquire the fat needed as the fuel, as well as whatever material they used as the wick. And if they were intending to venture deep into a cave then the amount of fuel and wicks required would have been substantial.

\subsubsection{Special techniques and styles}

There has been extensive analysis of the styles and content of the cave paintings and their possible changes over time, and although Leroi-Gourhan argued that there was a chronological sequence of four successive styles (Bahn 1998) (discussed in the following chapter), there have been no obvious patterns of major stylistic changes detected so far. Another interesting point is that all the paintings stand on their own and do not narrate any kind of story - they are just simple, individual representations, which are for the most part of large animals, usually herbivores (Leroi-Gourhan 1982). Also, unlike almost every other example of cave art, the images discovered in Chauvet Cave also contain shading and attempts at perspective (Chauvet, Brunel Deschamps et al. 1996).

Sometimes paintings were also made on surfaces that were first prepared by scraping away the uneven top layer to provide a smoothed out, flat area to draw on, or to make the image stand out more (Chauvet, Brunel Deschamps et al. 1996) - they created a kind of 'rock canvas'. In some cases, older paintings were scraped off before newer ones were painted on the surface, but in other examples new images were painted straight over the top of older ones - and in these situations the use of carbon-dating techniques have been made even more challenging than they already were.

As previously mentioned, outlines were sometimes drawn using charcoal before being painted over and the complete drawing filled in. André Leroi-Gourhan has shown that sometimes the artists would use stencils cut out of animal skin or wood to help trace the required outlines over the uneven rock surfaces (Ruspoli 1987). There are also examples of drawings that use a stippling effect whereby a series of large red dots form the complete image (Guthrie 2005). Chauvet Cave contains a good example of this style (see below) with the image of a bison being drawn. 


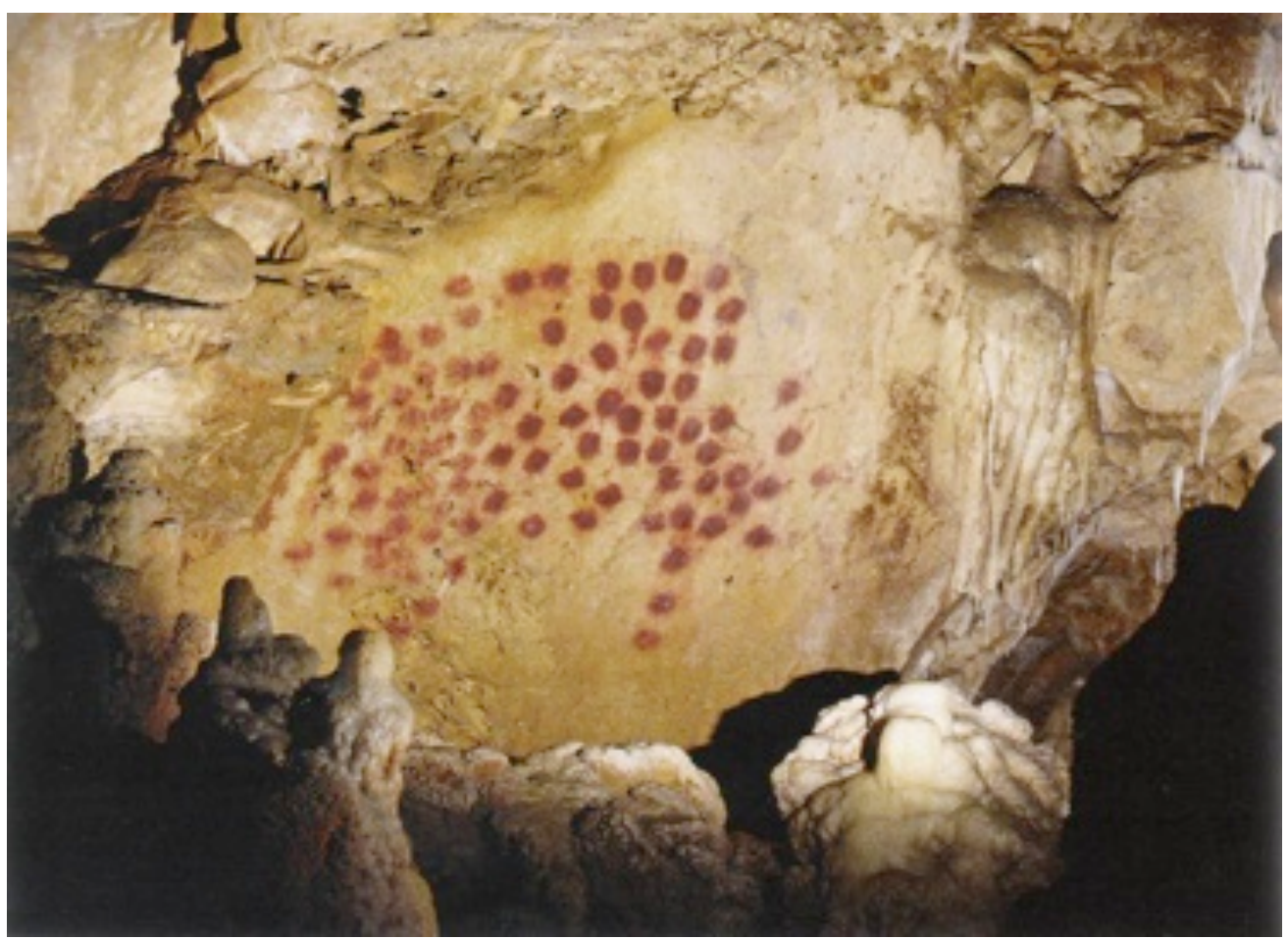

Figure 1.5 Representation of a Bison or Mammoth using a stippling effect, Chauvet Cave, France (Aurignacian) (Clottes 2008)

\subsection{Location}

\subsubsection{Inside the caves}

The location of paintings within the caves was varied and there seems to be no particular pattern. Some were created deep within the recesses of a cave where there would have been no natural light, whilst others were painted closer to the openings where some light was present. They have been found in big chambers as well as in the small ones. They were placed in open areas, as well as hardto-access locations that in some cases could only be accessed via traversing along dangerous ledges. Cave paintings have been discovered in areas used regularly (supported by other archaeological evidence) and those used only rarely. But despite this variation, all the paintings more or less contain the same sort of subject matter (Clottes 2008). Bahn points out that the art found in the open and readily accessible parts of the cave would have been 'public' and available for all to see, but the paintings produced in the very deep and difficult places to access appear to have been very much for 'private' appreciation and shared with very few others, if not solely by the artist alone (Bahn 1998) - some of the possible reasons for this will be discussed in the next chapter. 
It is important to note that not all the caves in any given area were used for painting and it has been argued that the choice of cave was not randomly made (Clottes and Lewis-Williams 1998). The topography of the cave was an important factor in determining where a painting was placed. A cave wall's size, features, and contours, as well as its general condition, would all determine how appealing its surface was for making the representations. The use of dim lighting to reproduce the conditions in which Paleolithic people painted, highlights just how significant the cave wall contours would have been - effects that are not present when modern and much brighter equipment is used in the caves. The unevenness of the rock surface that the artists painted on was utilised to add a three-dimensional appearance to what they had drawn (Lewis-Williams 2002). The artists often included in their paintings any unusually-shaped cracks or protrusions - as well as stalagmites (Bahn 1998) - that resembled or suggested some part of an animal's body (Clottes and LewisWilliams 1998). Leroi-Gourhan (1982) also observes that the Paleolithic artists often sought to express volume by making use of concave walls or ridges.

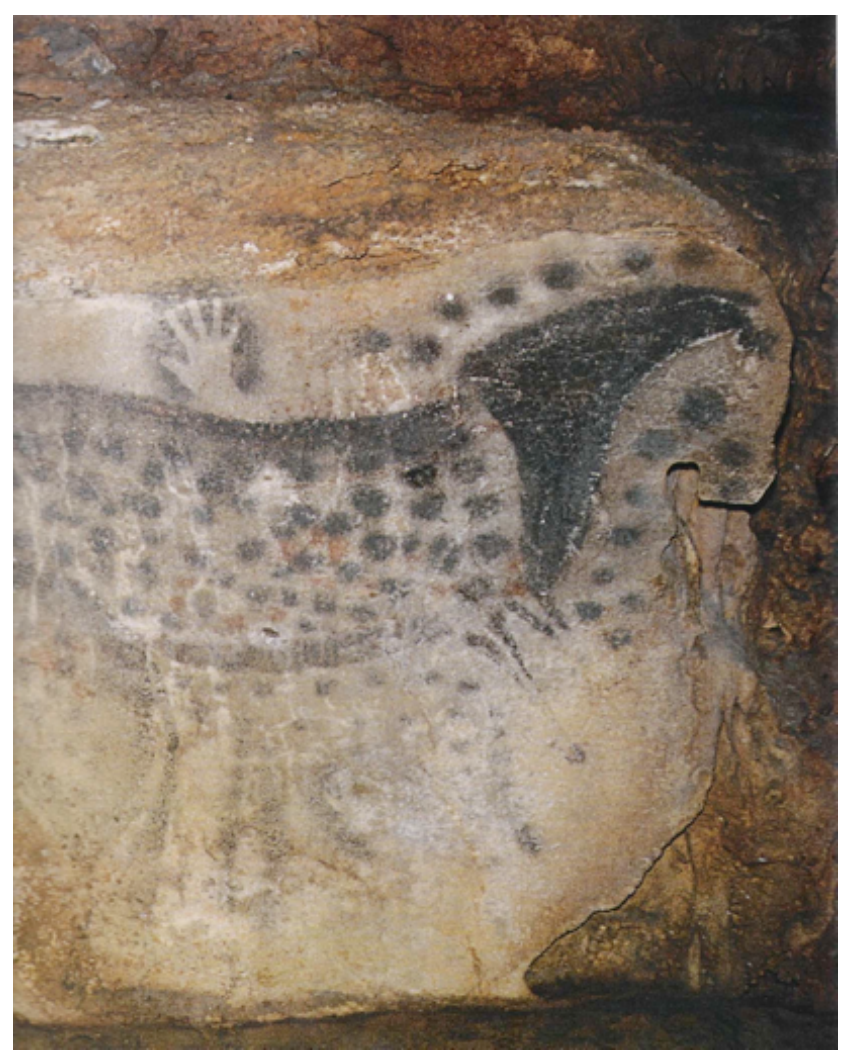

Figure 1.6 Black dappled horses drawn around a crack in the wall, Pech-Merle Cave, France-about 25,000 yrs old (Gravettian) (Clottes 2008)

Clottes in particular highlights the choice of location within the cave and how the artists used markings on the walls to stimulate what they drew: 
Everywhere, be it in the entrance zones or the areas at the end of the cave, the same attention is given to the striking topographic elements in the chambers and galleries; everywhere the artists made use of the hollows, the fissures, the contours of the walls. These parts of the cave either played a determining role in the production of the animal itself or were used as backdrops from which the animal was drawn as if emerging from a hole or the back of a gallery. (Clottes 2003, p. 149)

Analysis has shown that the use of deep caves for creating paintings occurred throughout the era of Paleolithic cave art, but it was more common towards the end of it (Clottes and Lewis-Williams 1998). Paintings have been found incredibly deep inside some caves, even as far away as a mile and a quarter from the cave entrance (Ruspoli 1987). The representations made in the deeper parts appear to have been produced in a shorter period of time than in the main caves, for they lacked the same amount of detail - as Guthrie says, "technically more demanding and time-consuming works are seldom located in deep caves. They are usually found in shelters or sunlit parts of caves" (Guthrie 2005, p. 37). Furthermore, they were mostly drawn by making use of the suggestive topographical features of the rock (Clottes and Lewis-Williams 1998). But the fuel for lamps could have been an expensive resource and the deeper one ventured into a cave, the more fuel was required. So it's possible that deeper cave art had to be done quickly and making use of suggestive contours would have resulted in a faster execution. As they became more efficient in later years, the fuel for lamps may have been produced in higher quantities at less cost.

\subsubsection{Outside of the caves}

Evidence of Paleolithic paintings have also been found in many rock shelters (Clottes and LewisWilliams 1998) - and drawing in these locations would have the added advantage of being accessible to daylight, but at the same time they were more open to the elements and so would be much less likely to have been preserved. And indeed there have been only the faintest tracings of paintings found (sometimes having been protected by habitation layers that built up on top of them) - this is almost certainly due to being exposed to the elements rather than it being the case that paintings were rarely made in these locations (Bahn 1998). Wall engravings have also been found at some of these shelters.

A number of discoveries have also been made of Paleolithic art produced in the open, on the sides of rock faces, where engravings into it have been made - for example at the site of Foz Côa in Portugal (Clottes and Lewis-Williams 1998). Due to erosion by the elements, this is the only type of exposed art that appears to have survived. So it can only be assumed that Paleolithic people also 
painted on rocks that were in the open-air, but the chances of having the right conditions to preserve any of it (and so proving this assumption to be correct) are likely to be extremely small.

\subsection{Subject Matter}

\subsubsection{Animals}

One of the main subject matters drawn on the cave walls is that of animals. From what has been discovered so far the choice of which animals to draw appears to be highly selective, with a majority of images being those of large herbivores. The most commonly depicted animal in Paleolithic cave paintings is the horse, although in some places there are many paintings of bovines, mammoths, ibex, deer, rhinoceroses, and others (Clottes and Lewis-Williams 1998). But it is also interesting to note which animals were very rarely or even never represented: fish, birds, and reptiles are almost non-existent (Leroi-Gourhan 1982). Chauvet Cave, containing the oldest known examples of cave paintings is a little different in subject matter in that although they are also of large animals, they are often of dangerous predators such as lions and bears (Chauvet, Brunel Deschamps et al. 1996), animals rarely drawn elsewhere.

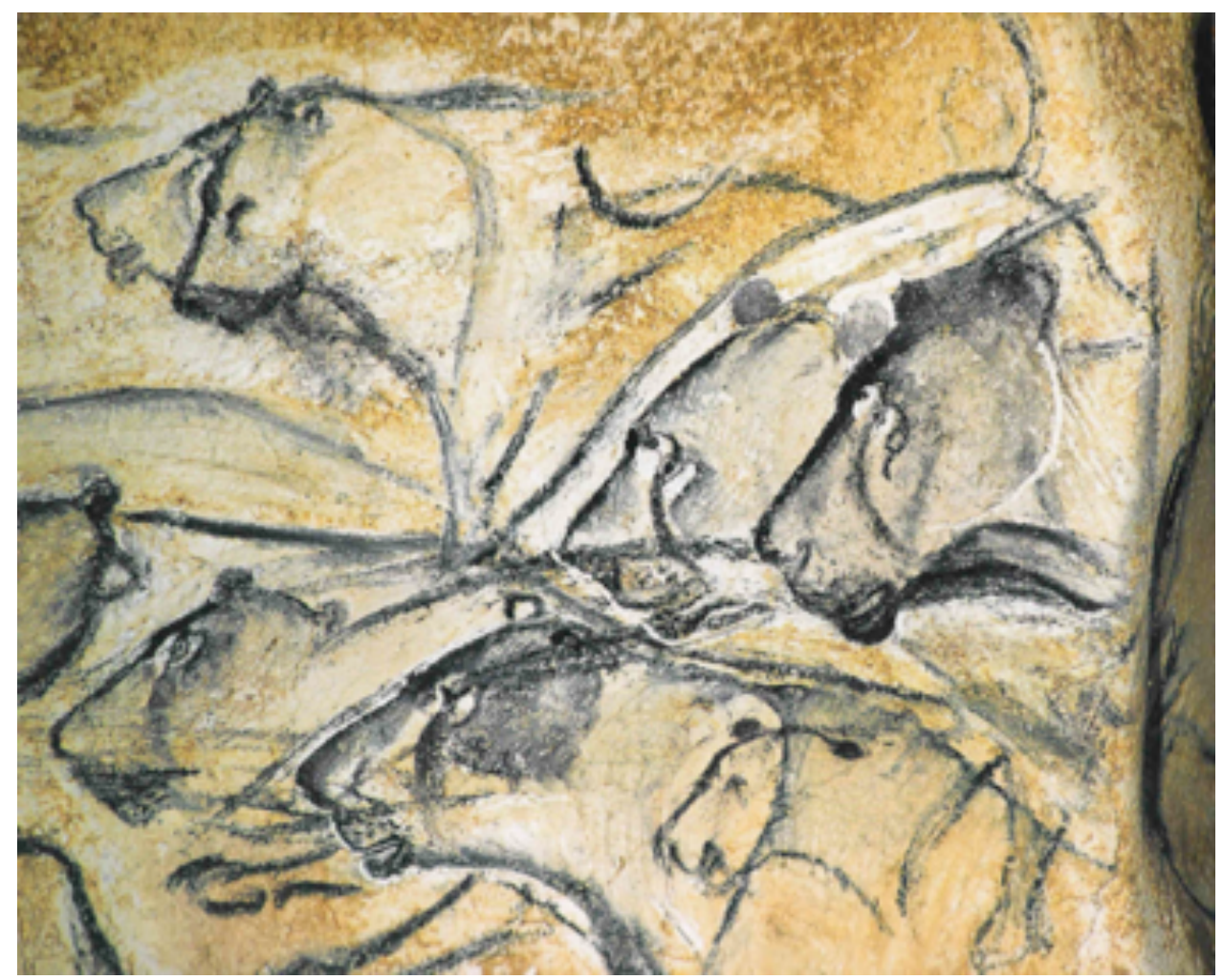


The choice of only certain animals has long puzzled archaeologists and several theories attempt to explain this (see chapter 2). One question that arises concerns the hunted animals represented on the cave walls: it was often the case that skeletal remains in the caves and surrounding areas show that the animals that were eaten were not those that were regularly drawn. For instance, in one area there may be many paintings of bison, whereas the main constituent of the local diet was actually ibex (Clottes 2008).

Another interesting observation is that the images were always drawn in side profile rather than in front or rear view. Additionally, they were also never drawn in context (e.g. standing on the ground) and are portrayed as being totally isolated from their surrounding environment, almost floating in space. These idiosyncrasies have thus also generated much debate.

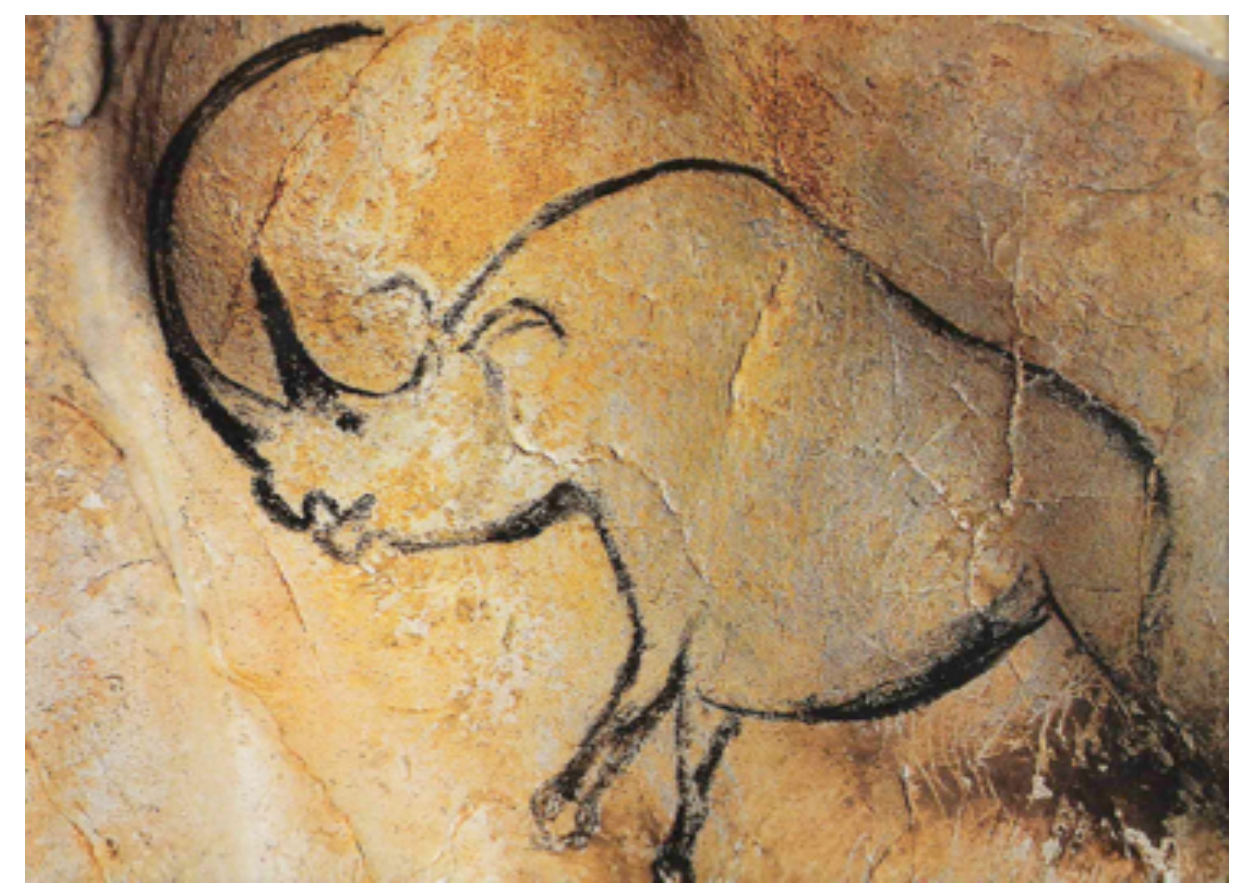

Figure 1.8 A female rhinoceros, Chauvet Cave, France (Chauvet, Brunel Deschamps et al. 1996)

\subsubsection{Humans}

Interestingly, there are very few (complete or detailed) paintings or engravings that are representations of humans (Clottes 2008) and those that have been found so far lack either significant detail or a naturalistic appearance (Leroi-Gourhan 1982). Human portraits have been discovered in a cave at La Marche in France dating to 14,000 years ago, but their authenticity has been questioned (Bahn 1998). This lack of human representation is quite the opposite of the more contemporary rock art found in the rest of the world. The most naturalistic representations of the 
human body are the many handprints of the Paleolithic artists: both 'positive' prints (using hands covered in paint) and 'negative' stencils (where hands were placed on the wall and then paint sprayed over the top of them) have been found in many caves and according to Clottes they date from the older time periods (Clottes and Lewis-Williams 1998; Clottes 2008). R. Dale Guthrie (2005) has extensively analysed the handprints found in caves and his statistical analysis indicates that the average age of the people who made them was between 10-16 years of age and were mostly males (although it wasn't exclusively the domain of young boys, and there are examples from older artists too. Sometimes the handprints had partial or whole fingers missing, possibly as the result of accidents, frostbite, or even mutilation, although in some cases fingers were deliberately curled up. There are numerous interpretations of these handprints, but one possibility is that they were nothing more than a kind of Paleolithic signature (Ruspoli 1987).

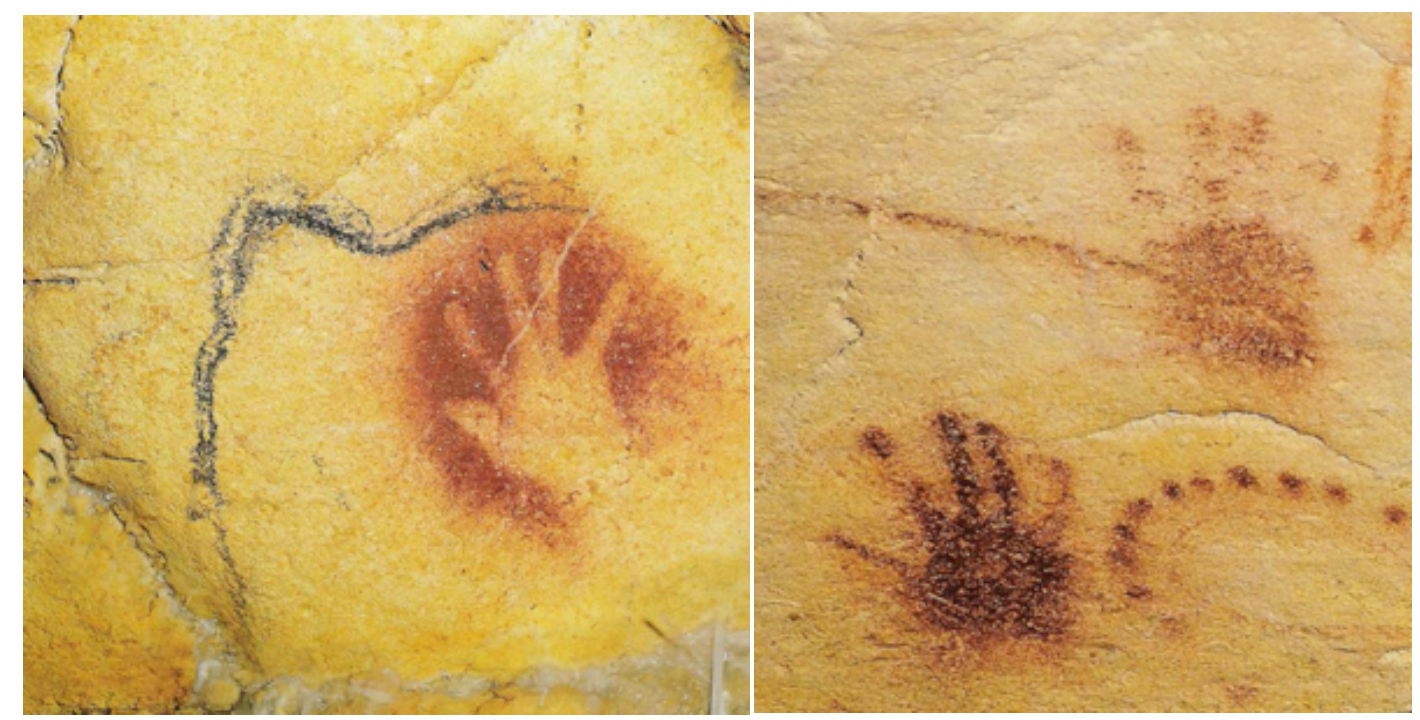

Figure 1.9 A hand stencil (left) and hand prints (right), Chauvet Cave, France (Clottes 2003)

There are also many drawings of pubic triangles and vulvas, indicating that the artists were preoccupied with thoughts of a sexual nature. There is also an engraving of a female holding a horn in her right hand (see below) - one of the very few representations of a complete human body made on the wall of a cave. It was originally painted in red (Clottes 2008). 

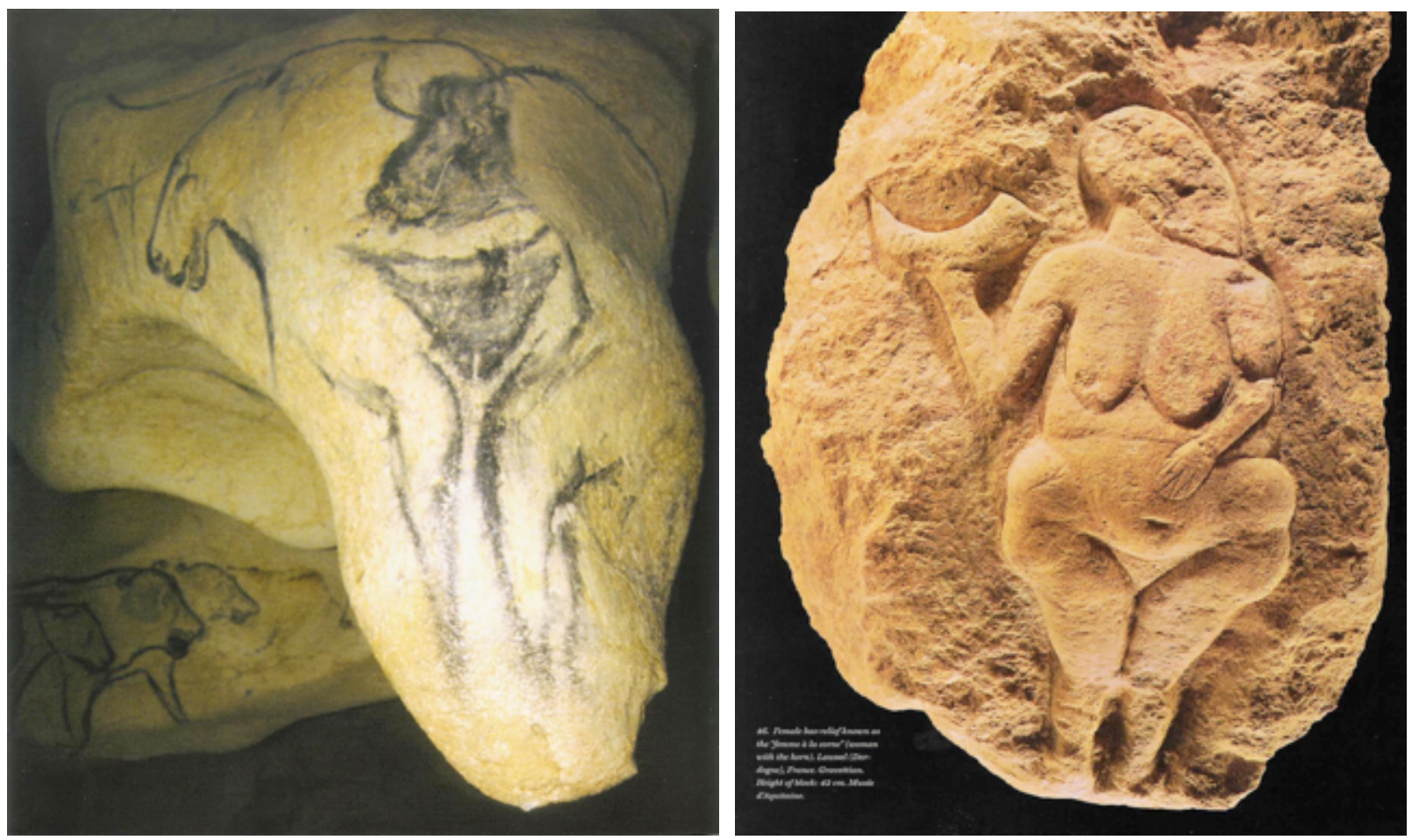

Figure 1.10 (above, left) Pubic triangle drawn on a stalactite-like protrusion, Chauvet Cave, France (Aurignacian) (Clottes 2003). The vulva mark was added on top of it by making a short, engraved vertical line (Clottes 2008)

Figure 1.11 (above, right) 'Venus with horn' bas-relief, Laussel Shelter, France (Gravettian) (White 2003)

Of the relatively few human representations, there are certain characteristics that they have in common: they were mostly incomplete drawings, and were often just a single part of a human body (usually female vulvas or handprints), and surprisingly they were quite unnatural in appearance this being a complete contrast to all the drawings that were made of animals. An additional type of human representation found in various locations was that of drawings composed of part man, part beast - interpreted as possibly supernatural beings. The lack of life-like human representations does raise the important question of why this would be the case and the next chapter discusses some possible explanations.

\subsubsection{Non-figurative markings}

Abstract marks and symbols have been found in most caves alongside the many paintings - almost every cave decorated with Paleolithic paintings so far discovered also has at least some amount of these strange marks, whilst at the same time there are very few caves that exclusively contain only these markings (Clottes and Lewis-Williams 1998). Lascaux Cave for instance, contains over 400 of these strange marks, whereas other caves in the surrounding area contain but a handful (Ruspoli 
1987). They have sometimes been drawn alone, but have also been drawn over the top of existing paintings. At times they appear to resemble shapes, but just as often it is not at all obvious that they resemble anything and any interpretation is usually quite subjective. There has however been recent speculation that they are an early form of writing. ${ }^{3}$ Alternatively, they might have been tally marks, or mnemonics of some kind.

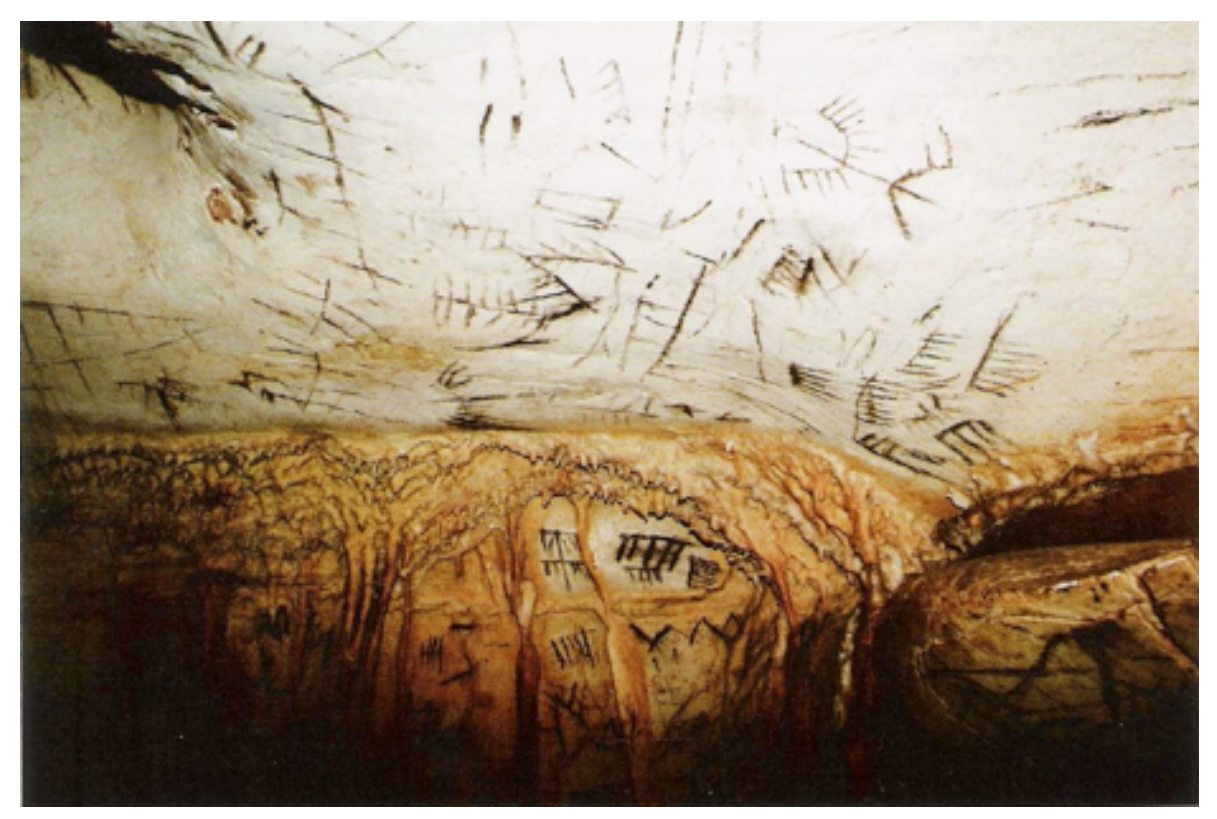

Figure 1.12 Abstract markings, Cueva de la Pileta, Spain - approximately 27,000 years old (Spivey 2005)

Whilst these marks continue to remain a mystery, red and black spots, as well as squiggles, and single or multiple lines have also been found. The lines are often short and straight - in parallel groups, or sitting on their own; sometimes they form a small grid pattern. The dots are often superimposed on top of the cave paintings, but others are on their own and sometimes form approximate shapes that resemble an animal (Chauvet, Brunel Deschamps et al. 1996). The red dots are sometimes in parallel rows and this has led some to identify them as representing the blood trails of wounded animals (Guthrie 2005), but others have read much more into them and have used them to support their own mystical theories of the origins of Paleolithic cave art (see chapter 2). It is not unreasonable to assume that at least some of the small lines drawn on the paintings are representing spears.

The claw marks of cave bears have often been found on the cave walls - along with even the small scratches of bats (which have in some cases been misinterpreted as having been carefully made by

\footnotetext{
${ }^{3}$ See New Scientist Magazine, $20^{\text {th }}$ February 2010 for early coverage of research being carried out by Genevieve von Petzinger of University of Victoria, British Columbia, Canada. Papers in Antiquity and Journal of Human Evolution forthcoming.
} 
the artists). These claw marks have at times been incorporated into the abstract marks made by the people who saw them (Chauvet, Brunel Deschamps et al. 1996; Bahn 1998) - and to some extent, this ties in with the way that the artists often made use of the natural contours in the caves when creating their paintings.

\subsection{Representations other than paintings within the caves}

Engravings were common in Paleolithic art and many have been found in the caves alongside the paintings. They could be made using anything from a handaxe, to flakes of flint, or a piece of wood (Ruspoli 1987) - it just depended upon how hard the rock was in the cave that they wanted to cut into. Using fingers to scratch the surface would have been the easiest method of creating a mark or a small image on a cave wall and if the wall was made of a soft enough substance such as clay, then a sufficiently deep marking could be made without the need for any tools (Bahn 1998). Many examples of these images made by fingers have been found in the caves including at La Clotilde in Spain (Clottes and Lewis-Williams 1998). The image below is of a horse that was made using a combination of engraving tool and fingers. It was produced on a soft surface and the area was first scraped clean (Clottes 2008).

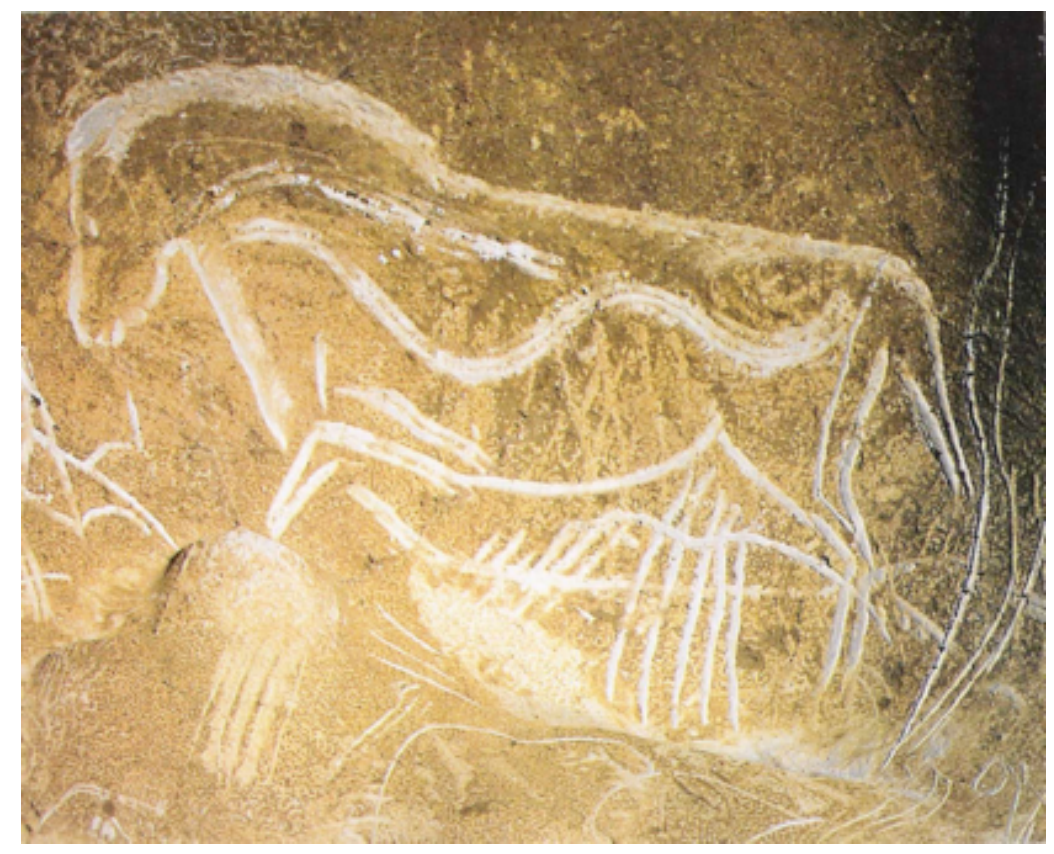

Figure 1.13 An engraving of a horse in Chauvet Cave (Aurignacian) (Chauvet, Brunel Deschamps et al. 1996; Clottes 2008)

The dating of an engraving on a cave wall is much more difficult than a painting, but it is still sometimes possible. Unfortunately, the age of engravings and depressions are impossible to 
calculate using radiocarbon dating techniques due to their lack of organic material, so a contextual methodology including the dating of the type of animal depicted has to be used instead. Like paintings lacking organic material, some guidance as to their possible age can be derived in cases where either a habitation layer has built up beside it, or if any organic material - or even calcite deposits - have formed on top of it, as this will provide an 'at least as old as' date (Ruspoli 1987). And as has previously been mentioned, the occasional carbon deposit left by the wiping of torches on top of an engraving can at least provide a minimum age.

A limited number of examples of pieces of clay moulded into the shape of animals have been discovered, for instance the two bison from a cave at Ariège in France that are dated to around 1712,000 years ago (Bahn 1998; Clottes 2008). Further Paleolithic images have also been found usually lying on cave floors - that have been engraved or painted on slate plaquettes; in Parpalló Cave in Spain (which was occupied continuously from 29,000 until 11,000 years ago), over 5,000 of these plaquettes have been discovered (Bahn 1998).

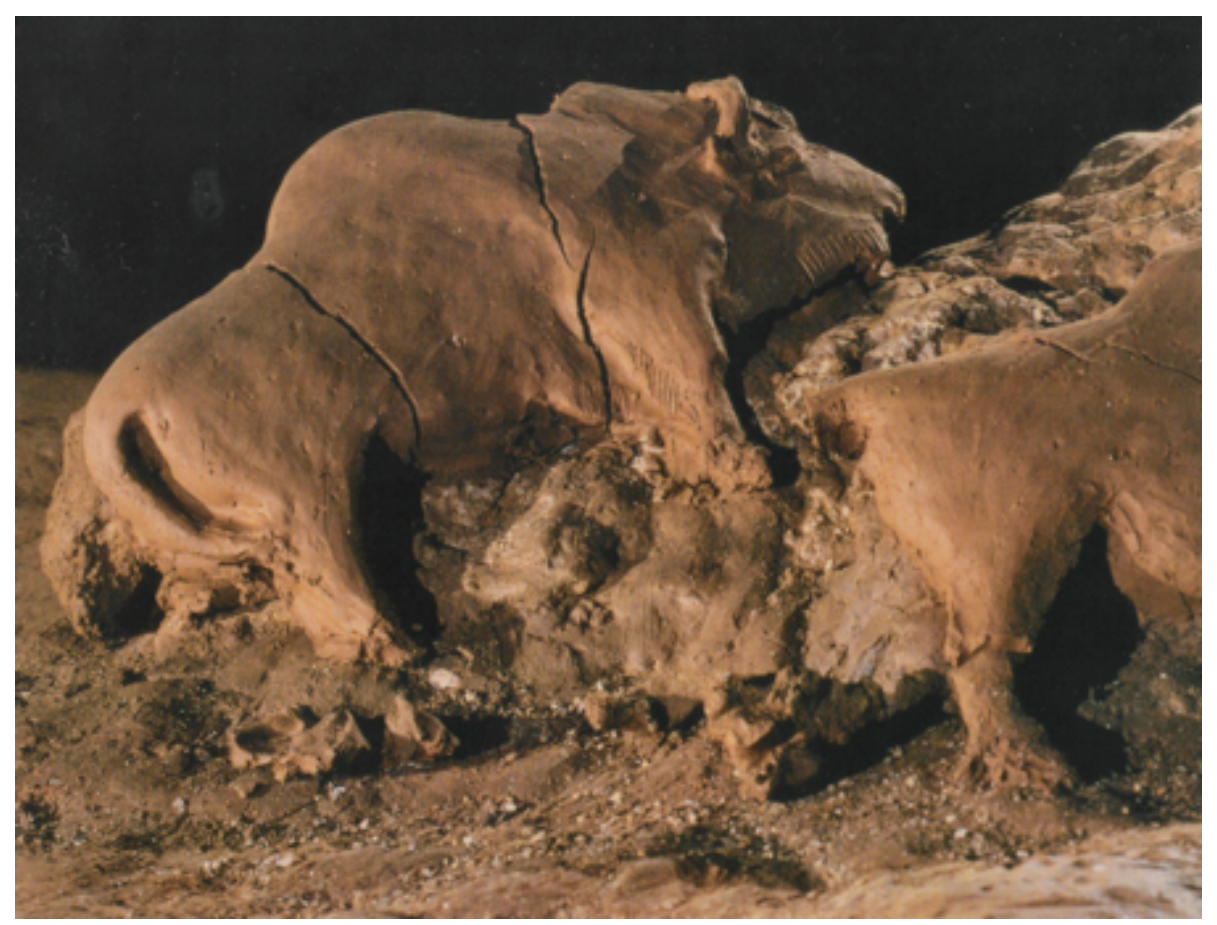

Figure 1.14 Bison moulded from clay, Le Tuc d'Audoubert Cave, Ariège, France (Middle Magdalenian) (Clottes 2008)

\subsection{Quality}

The quality of the representations is astounding and as Ruspoli (1987) points out, it wasn't until the era of the Greek civilisation that this level of naturalistic accuracy was once again attained. One 
question that arises, given the sheer quality of Paleolithic cave paintings, is to ask why there aren't any examples of poor quality art, i.e. badly drawn animals. Part of the answer may lie in the fact that it seems reasonable to think that only a tiny fraction of the parietal Paleolithic art that existed has survived - and due to where they were situated, it would have been the cave paintings that had the best chance of avoiding destruction (Guthrie 2005). Whilst purely speculation - and more on this subject is discussed in the next chapter - one possibility was that only proficient artists were 'allowed the honour' of drawing in caves: one had to practice outside first, and these primitive examples have been lost to the elements.

Many of the paintings and engravings contained far more detail than what would have been required for a simple representation of an animal. The drawings below are from Niaux Cave in France and form part of a larger panel that contains eight bison and two ibex, and which was drawn in charcoal. Lines have been included that draw in the manes and beards of the animals, and there are also lines on one of the bison and ibex that appear to be barbed arrows (Clottes 2008). The amount of detail in the eyes even includes drawing in their pupils.

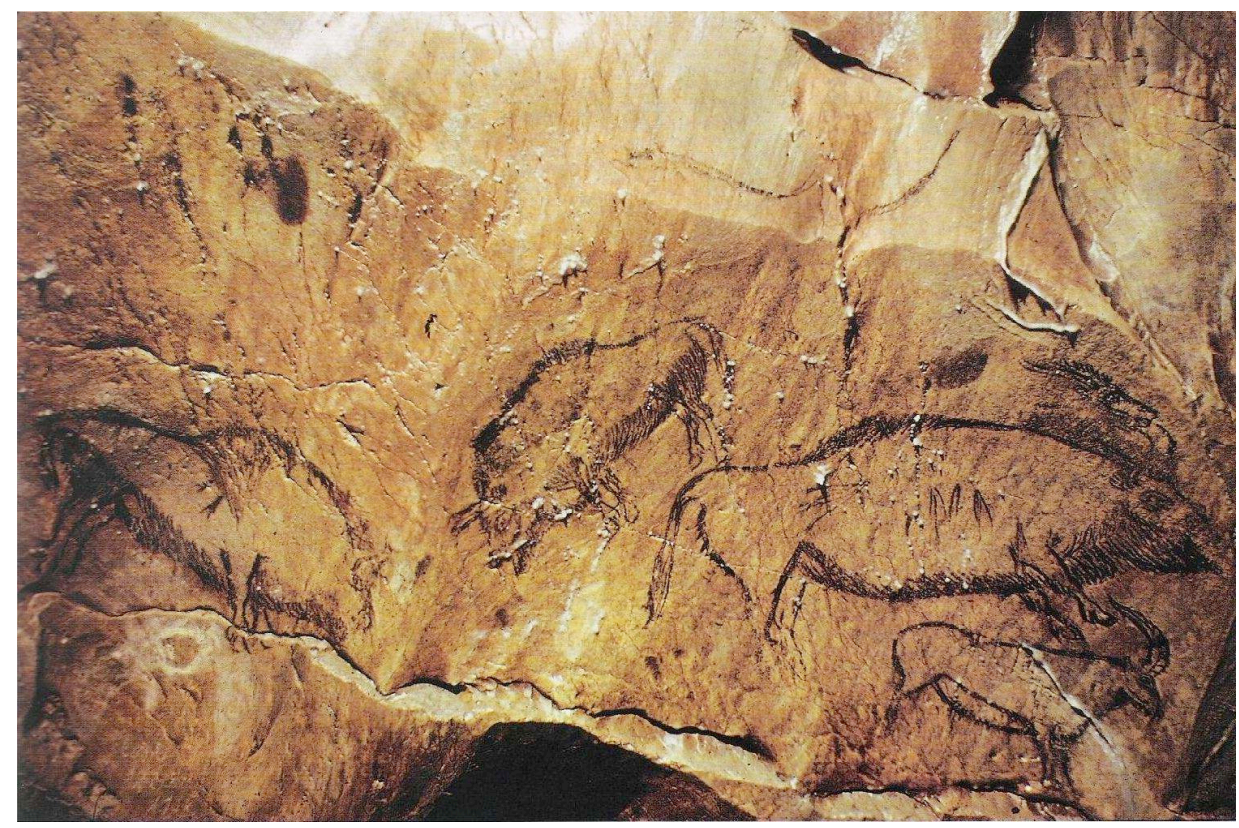

Figure 1.15 Bison and Ibex approximately 13,000 years old (Middle or Upper Magdalenian), Niaux Cave, Ariège, France (Clottes 2008)

It does seem highly likely that practice must have been needed before these paintings were made there may have been some sort of apprenticeship, or possibly it was simply by a process of trial and error. Interestingly, Jean Clottes (2008) highlights the fact that engravings on stone plaques are comparable to wall art, but often are of a lower quality than the engravings found on the cave walls 
- so it's possible that these were used to practice on before being allowed to venture into a cave to decorate it.

Whilst a lot of the paintings would have taken perhaps several hours to draw, the bigger images would have taken a considerable length of time to create them. Indeed, based upon the experiences of reproducing them at 'Lascaux II', the replica Cave of Lascaux that was created for tourism, it is speculated that some of the larger, detailed paintings would have taken several days to complete (Ruspoli 1987).

\subsection{Dangers}

It is a misnomer when people of the Upper Paleolithic are referred to as 'cave men'; evidence indicates that Cro-Magnons usually inhabited only the outermost part of caves due to the inherent dangers and the lack of natural light. Shelter from the cold northerly winds would have been the main reason to venture just inside the openings. Archaeological evidence suggests that it was much more common for people of the Upper Paleolithic to make use of a rock overhang (also known as an 'abri') as a natural shelter from the elements (Guthrie 2005).

Venturing into a cave often involved significant risk. Cave bears - bigger than modern-day bears were a constant threat when entering a cave up until their demise approximately 27,500 years ago. Their claw marks often cover the cave walls and their footprints and large depressions that were their 'nests' mark the softer cave floors (Guthrie 2005). The floor of Chauvet Cave was littered with the bones and skulls of cave bears and it was not uncommon for these bears to die during hibernation (Chauvet, Brunel Deschamps et al. 1996). In Chauvet Cave the skull of a cave bear was even found that had been deliberately placed on a fallen rock (see figure 2.3), although the motivation behind this act will likely never be known (Chauvet, Brunel Deschamps et al. 1996). Interestingly, Guthrie hypothesises that a possible explanation of why there is a lack of evidence for the use of caves prior to 32,000 years ago may be due to the presence of cave bears ${ }^{4}$ (Guthrie 2005). Furthermore, accessing the more remote and deeper areas of the caves to make paintings might have required the traversing of dangerous ledges or the negotiation of narrow passages with steep drops off to the side. Entering caves often involved significant risk. Prima facie, the motivations for entering caves must have been strong.

\footnotetext{
4 Fossils show them in abundance 60-30,000 years ago, but then their numbers declined rapidly, most likely due to climate change (and they became quite rare by approximately 25,000 years ago). With the potential danger of encountering such a large and dangerous creature, Cro-Magnons would have been wise to avoid venturing into the caves; (Guthrie 2005)
} 


\subsection{Climate and preservation}

Climate played an important role in both the production as well as the destruction of cave art. Archaeological finds show that during the colder parts of the Upper Paleolithic, and when encountering severe winters, rather than just seeking temporary shelter in the outer areas of caves, Cro-Magnons sometimes retreated into them and lived there for extended periods (Ruspoli 1987). Furthermore, many of the caves were south facing as they were shielded from the cold northerly winds (Guthrie 2005). Unlike the caves, the majority of decorated rock shelters are in southerly locations of Western Europe where it would have been warmer (Clottes 2008). But rock shelters did not supply adequate protection from the colder weather, so the people of the Paleolithic would have made use of the added shelter of caves in the more northerly locations. Also, during winters, when it was dark for long periods, there would have been more time available for cave painting, but resources would have been scarcer.

One question that arises is whether there is any connection between the increased production of cave art and the climate changes that forced our ancestors to either retreat into caves when it was colder, or to make more use of rock shelters during the warmer time periods. The abundance of paintings around certain dates appears to support archaeologists' predictions that cave art production would increase during periods when the climate changed rapidly, causing the Paleolithic inhabitants to continually relocate. This situation occurred with the advent of the last ice age, which made previously inhabitable land uninhabitable, and then as it receded the opposite situation occurred (Pike 2008).

But the dramatic changes in climate almost certainly destroyed many sites of cave art. The cooling temperatures of the Ice Age would have resulted in advancing glaciers that crushed any caves in their path, including those that contained paintings - and then when they receded the areas would have been flushed by large amounts of run-off water (Guthrie 2005). With the rise in sea levels at the end of the Ice Age, areas of Paleolithic habitation that were once along the coastline became totally submerged and in some cases are located many miles out to sea - the Cosquer Cave is some thirty seven metres below the sea's surface, for example.

The type of rock present in an area was also important, as the thick limestone of Western Europe crucially led to the creation of the many caves and overhangs that were utilised as shelters (Guthrie 2005). The rock's surface also needed to be stable: some types of rock will flake off over time and 
the paintings will slowly crumble - sometimes small pieces of coloured rock have been unearthed at the base of walls indicating the probable existence of a painting many years earlier.

Environmental factors had a major effect on the preservation of Paleolithic art, with special climatic and geological conditions required for the preservation of the pigments. Any exposure to the wind or rain over many thousands of years would severely limit the chances of a particular painting surviving intact and thus a dry climate helped. But in many parts of Africa for instance, this becomes irrelevant because a lot of the rock is of a sandy base and is not stable enough to allow paintings made on it to survive for any extended length of time. More relevant to caves is the risk of calcium deposits slowly building up over time that can gradually destroy or cover paintings and engravings. Water running through cracks, underground rivers, and issues of flooding also contribute to the destruction of cave art. An abundance of plant growth is another factor: it can destroy paintings out in the open but in some cases it can offer a level of protection to some engravings.

The relatively stable climate and humidity of the inside of a cave provides almost perfect preservation conditions. But a cave's entrance is much less favourable and a combination of steady moisture, and temperatures dropping below freezing, can cause the surface stone to crack and fall to the ground over time. It is occasionally possible however, for part of a painting to actually be preserved by this process: if it broke off and fell face-first onto the ground, and then the sediment surrounding it was favourable to preservation (Guthrie 2005). The darkness of a cave also helps in preservation, as the sun's ultraviolet light would gradually break down the chemicals that make up the pigments used to create the paintings. Another cause of damage to paintings comes from animals, so if none ventured into a particular cave then it would remove the chance of damage from any abrasion or repeated rubbing; claw marks and bat droppings have been found on many cave walls, as previously mentioned.

But the stable conditions within a cave are fragile. Once a new discovery is made, the movement of only a few people in and out of a cave can quickly disturb the stagnant air within it. After only several visits changes begin to occur and it does not take long before noticeable deterioration in the quality of the paintings is observed (Bahn 1998). Effects resulting from the additional moisture from human breathing, temperature changes, and general movement of bodies and equipment mixes up the previously motionless air. Furthermore, visitors to a cave (including animals) can introduce pollen and algae that can begin growing on the walls, causing further deterioration (Ruspoli 1987). 
Thus, caves with entrances that have been totally blocked off by rock falls provide the best chances of preserving paintings.

So there are many factors governing the preservation of a Paleolithic painting or engraving over thousands of years. This probably explains why most have been found in the caves and shelters of Western Europe yet there is almost no evidence of any being produced out in the open. It is therefore highly likely that the cave art discovered so far is a grossly distorted selection of all the Paleolithic art that once existed. ${ }^{5}$ Caves and shelters provide the most significant portion of the Paleolithic paintings found, not because that is where it was mostly produced, but because that is where the vast majority have been preserved - as Guthrie states, "we can safely say that cave art is a small sliver of the Paleolithic art that existed and was preserved only because of its protected location" (Guthrie 2005, p. 35). The number of caves and shelters - possibly containing art - that have been destroyed by climatic effects will never be known.

\section{OTHER ANCIENT ART}

Whilst the majority of the earliest known art has been found in the caves of Western Europe, it is still worth briefly surveying what other ancient art has been found. The craftsmanship involved in creating some of these pieces of artwork provides a further indication of just how technically advanced our ancestors were in their artistic behaviour. I will now summarise the most significant examples.

\subsection{Other Paleolithic images and representations found in Western Europe}

Paleolithic images are not solely restricted to the walls of caves and rock shelters of Western Europe, they are also found in the form of beautiful engravings on osseous artefacts such as weapons, and these have been found out in open areas as well as near locations of parietal art (Bahn 1998). Other Paleolithic representations in the form of carvings of animals, 'Venus' figurines, and decorated spear throwers have all been found (Ruspoli 1987) - so many, that they number in the thousands (White 2003). They made use of bone, antler, stone, and ivory in the creation of these

\footnotetext{
5 Guthrie provides a nice diagram of this; see page 35 of Guthrie, R. D. (2005). The nature of Paleolithic art. Chicago, University of Chicago Press.
} 
objects, and would have undoubtedly used other, perishable materials, such as wood and bark, clay, and dried mud, that have long since disappeared.
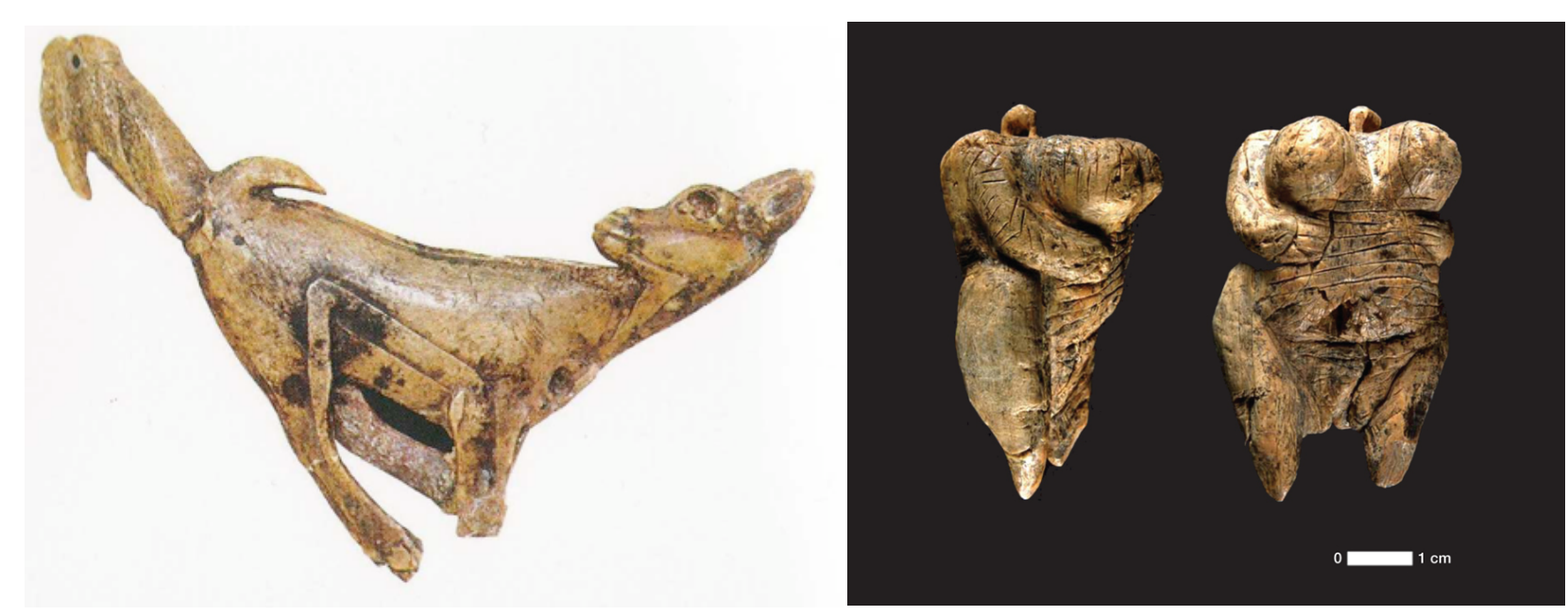

Figure 1.16 (above, left) Decorated spear thrower made from reindeer antler, found in a cave at Ariège, France (Middle Magdalenian) (Clottes 2008)

Figure 1.17 (above, right) Venus of Hohle Fels figurine made from mammoth-ivory, recovered from Hohle Fels cave in southwestern Germany. It is at least 35,000 years old (early Aurignacian) (Conard 2009)

Whilst a diverse amount of portable Paleolithic art has been found, there is a small portion of it that is of particular importance because it represents some of the oldest art known to date - on a par with the oldest examples of cave art. The most well-known of this group are the Venus figurines: small statuettes of female bodies with exaggerated breasts and hips. There are also carvings of mysterious 'anthropomorphs' (half human, half animal). Some of the best examples of this portable art are the many objects found in Germany, and in particular from the caves of Vogelherd and HohlensteinStadel which are from the same Aurignacian period as the paintings found at Chauvet Cave (Chauvet, Brunel Deschamps et al. 1996). The oldest of these is the Venus of Hohle Fels (see above) that has been dated to 35-40,000 years old, currently the oldest known example of Paleolithic art in Western Europe; it was found almost complete, with only the left arm and shoulder missing (Conard 2009). 

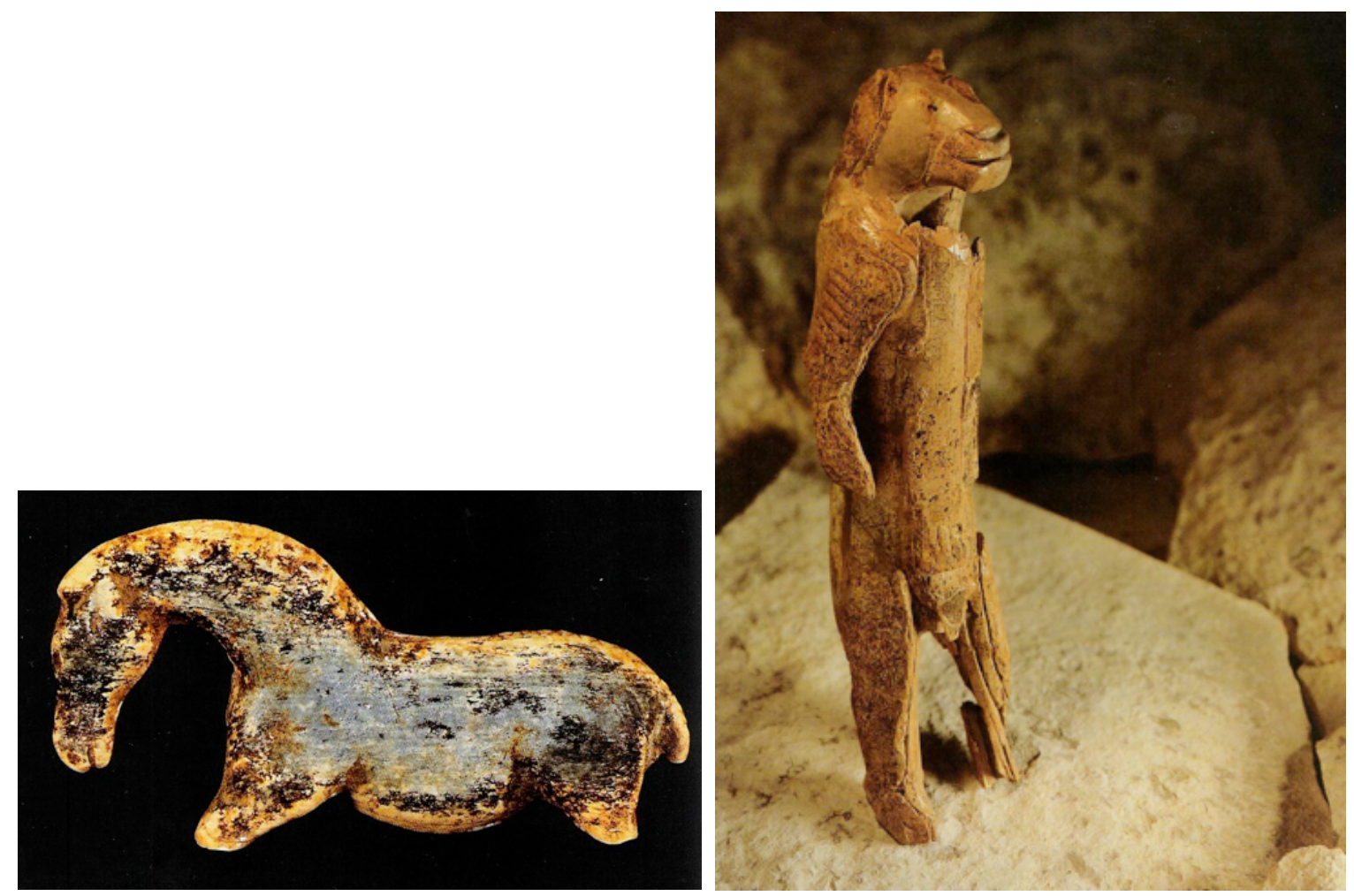

Figure 1.18 (above, left) 'Vogelherd horse' 33,000 years old (Aurignacian), made from mammoth ivory, found in the Cave of Vogelherd, Germany (White 2003)

Figure 1.19 (above, right) 'Lion man' figure made from mammoth ivory, 30-34,000 years old (Aurignacian), found in Stadel Cave, Hohlenstein, Germany (White 2003)

Another form of Paleolithic art is the Petroglyph, or rock engraving. Unfortunately, we will never know how prevalent Petroglyphs were during the Paleolithic because they will have been weathered away by the elements, or become covered by plant growth such as lichen and moss, or by calcite deposits, making them extremely difficult to detect (Bahn 1998). The dating of Petroglyphs, like engravings, can be problematic. Date estimates are unreliable, often varying wildly; but they do exhibit the same Paleolithic look and style.

\subsection{Other regions with Paleolithic images}

Whilst prehistoric images have been discovered in many other parts of the world, there are often many doubts concerning their age - unlike the paintings and engravings of Western Europe that have been both universally accepted and verified by dating methods as being Paleolithic (Bahn 1998). Furthermore, what has been found so far does not compare with the sheer quality of the examples found in Western Europe. The majority of the non-European images are in the form of engravings on rock that are extremely difficult to date (Clottes 2008). They are also mostly located 
out in the open or at rock shelters, rather than within caves (Lewis-Williams 1983). Wild claims concerning age have at times been made, for example about the thousands of cup marks chipped into the rocks at Jinmium in Australia: although purported to be up to 70,000 years old, experts believe that they are anything from at most 20,000 to possibly as little as 2,000 years old (Bahn 1998).

I will now briefly discuss some of the best and oldest examples of prehistoric images from outside of Europe that have so far been discovered. In Australia, the dry climate allows the preservation of paintings - unlike the glacial winds and rain that those in Western Europe were exposed to (Ruspoli 1987). Australian rock art was originally thought to be relatively recent, but its age was substantially revised backwards when in 1929 engravings at Devon Downs Shelter were found beneath 4 metres of accumulated debris - they are currently dated to approximately 13,000 years ago (Bahn 1998). Unfortunately, many of the aboriginal paintings were drawn with non-organic mineral pigments such as pure ochre and therefore cannot be dated accurately using the radiocarbon method. Rock paintings discovered at Judds Cavern in Tasmania have been dated to 9-10,000 years ago from organic materials found in the paint, possibly human blood (Bahn 1998). There is speculation that the many petroglyphs found in Australia have a long history, but dating them is extremely problematic and at present there are no techniques for reliably doing this (Clottes 2008).

Still in Australia, pigments including ochre (which could indicate painting) have been found in Northern Territory and dated to about 60,000 years ago (Bahn 1998), based upon the age of the habitation layer in which they were discovered. But this date is highly debatable because it conflicts with more robust data that suggests anatomically modern humans first inhabited Australia much later than this - although some archaeologists do claim that the first humans arrived between 7050,000 years ago (White 2003). So whilst there are many separate archaeological finds supporting a date of 40-50,000 years ago for Australia's first human inhabitants, the older dates associated with the artefacts and ochre pieces found at the Malakunanja II and Nauwalabila I rock shelters are much less reliable. The dating of the items discovered in these locations rely on comparative dating methods rather than the more reliable radiocarbon analysis and as such, any natural or unnatural disturbance of the surrounding sedimentary layers could have greatly distorted the date approximations. Furthermore, whilst these pigments might have been acquired for creating paintings, they may equally have been used for other purposes such as body decoration (Bahn 1998) or for dressing wounds, as has previously been mentioned. The bottom line however, is that any claim made for Australian images being as old as those of Western Europe is certain to be a contentious one. 
Elsewhere, rock shelters located at Bhimbetka in India provide evidence of the earliest known inhabitants in the region - at least 100,000 years ago - and they are decorated with paintings dating back to approximately 10,000 years ago (Clottes 2008). In South America, the caves of Toquepala contain paintings dated to at most 10,000 years ago based upon woollen brushes impregnated with ochre (Bahn 1998). There are also paintings at Perna in Brazil that have been covered by occupational layers dating to 10,000 years ago, so they have to be at least as old as this date. And at Pedra Furada, also in Brazil, paintings in rock shelters have been dated to approximately 11,000 years ago (Bahn 1998).

In Africa, the Apollo 11 shelter in Namibia contains the oldest known evidence of rock art in Southern Africa, with charcoal taken from the same layer as the painted stone fragments found having been dated to at least 19,000 years ago and even possibly as old as 27,500 years ago (White 2003). Only seven small pieces of painted rock were found, so not very much can be deduced about either the style of art or the what was being represented, although two of the pieces fitted together and rough outlines of parts of animals could be surmised (Lewis-Williams 1983).

\subsection{Other forms of early art}

One of the earliest forms of 'art' so far discovered are the beautiful Acheulean handaxes of the Lower Paleolithic from up to 1.4 million years ago. These handaxes appear to have been made for purely aesthetic reasons as they were either too large to be practical, symmetric beyond requirement, or extremely polished - all of which are suggestions that their overly crafted shape and form is the manifestation of some sort of primitive artistic intent (Bahn 1998). This interpretation, however, is extremely controversial. It has even been suggested that the function of these handaxes was to attract sexual mates. ${ }^{6}$

Due to the sheer amount of pigment that has been found in many habitation areas, including ochre, it is reasonable to assume that some amount of body-painting also took place (Ruspoli 1987). Smoother areas of the ochre suggest that it had been rubbed against a soft surface, possibly skin (Bahn 1998).

\footnotetext{
${ }^{6}$ See Kohn, Marek, and Steven Mithen. (1999) "Handaxes: products of sexual selection?" Antiquity. 73: 518-26.
} 
Another way of decorating the body was by using jewellery, which was often made by stringing together perforated shells, teeth, or bones; other materials were most probably used as well, but they would not have been preserved. The remains of Paleolithic jewellery are plentiful and have been found in many parts of the world as well as in Western Europe (Guthrie 2005). The oldest known jewellery has been found in Blombos Cave in South Africa, where perforated shells have been found that are dated to 75,000 years ago (Henshilwood, d'Errico et al. 2004). Interestingly, shells have been found - some perforated - hundreds of kilometres from their source, indicating that they were possibly traded (White 2003) and therefore had symbolic value.

Other forms of art such as song, dance, and narrative would almost certainly have been a part of rituals and general entertainment, but they do not leave any fossil trace, so it's impossible to be sure of any exact dates due to the lack of hard evidence. Ethnographic comparisons with modern-day hunter-gatherers suggest that these art forms were a significant aspect of Paleolithic life.

When instruments rather than just singing were involved, music making did leave traces in the archaeological record. But proving the use of some of these basic instruments is difficult unless they were specifically engineered for the purpose of making music. Bones, jaws, and skulls, etc, may have been used for percussion (Ruspoli 1987), but even if discovered, their function as being something other than just a bone, jaw, or skull would be extremely hard to determine. Very occasionally, musical instruments such as hollow bird bones with holes in them have been found (Bahn 1998), such as the flute from Hohle Fels (made from the wing bone of a vulture) which was only discovered in Germany in 2008 and has been dated to approximately 35,000 years ago (Conard, Malina et al. 2009). Other flutes dated to around this time have also been unearthed including several at Isturitz Cave in France dated up to 34,000 years ago (White 2003). But these fossil 'finds' can be misinterpreted: a purported Neanderthal flute made from the bone of a cave bear and found in a cave in Slovenia in 1995 has been dated to approximately 43,000 years ago. Its use as a musical instrument is hotly disputed however, as there are only two holes in the bone fragment, and it has been claimed that they were actually made by a bite mark produced by the chewing action of a carnivore (D'Errico, Villa et al. 1998).

\subsection{More contemporary cave and rock art}

Although this thesis is only focusing on the earliest known examples of cave and rock art from approximately 40-10,000 years ago, prehistoric paintings have been produced over many thousands 
of years, lasting through until relatively recently. It is interesting however, to take note of some of the more prolific indigenous art and what can be learnt from it.

Prehistoric rock art, including paintings created several thousand years ago by the San people (also referred to as Bushmen) of Southern Africa, are in abundance. In particular, thousands of painted images have been found at Drakensberg in South Africa. They differ in several notable ways from what has been found in Europe: they are located out in the open in rock shelters and the paintings are, in general, much smaller (Lewis-Williams 1983). Also, whereas the European drawings are mainly stand-alone, naturalistic representations, those of the San depict scenes that are telling some sort of story - they are drawn in context. This is also true of Australian Aboriginal art. And importantly, unlike the cave art of Western Europe, human representations are present on a regular basis in the more contemporary cave paintings found in these other regions of the world (Clottes and Lewis-Williams 1998). They also depict a much wider variety of animals including many of smaller ones such as snakes and birds.

\section{ANALYSIS}

The discovery of cave art in Western Europe raises many interesting questions and has also forced archaeologists and anthropologists to rethink many of their previous conceptions about the cognitive evolution of humans. In this next section I examine some of the main issues surrounding western European cave art.

\subsection{Why Western Europe?}

Whilst ancient drawings have been found in many other parts of the world, the sheer quantity, quality, and much older age of what has been discovered in Western Europe raises many questions. One has to wonder why the Paleolithic art of Western Europe appears to be so prolific when compared to what has so far been discovered in the rest of the world combined.

An initial response points to two possible answers. The first is that anatomically modern humans in Western Europe suddenly developed an art-producing capability approximately 35-40,000 years ago - a theory generally referred to as the 'Creative Explosion', and something that I will discuss in more detail shortly. A second explanation could be that local conditions in Western Europe resulted 
in the preservation of a much higher proportion of art than in any other location in which it was created. There is a degree of truth to this claim, because due to the geology of Europe it has a much higher number of caves (Guthrie 2005) and any art created in them would have been well protected. Much of the art produced outside of Europe on the other hand, was made out in the open rather than in caves and so was much more likely to have been destroyed (Clottes 2008). I discuss this issue of taphonomy later on in this section. But this explanation does still not appear to account for there being such an extreme dearth of similarly-aged parietal art worldwide.

There is also however a third and more plausible explanation, which is that this is simply where it has been looked for the most. Western Europe contains a much higher concentration of archaeological research than in any other part of the world. Furthermore, the region is also heavily populated, increasing the possibility of chance findings by members of the public or dedicated spelunkers. This third possibility leaves open the prospect that there is much more Paleolithic art yet to be discovered in the rest of the world, but until these regions have been more thoroughly explored we will not know for sure. Even then, time may not tell.

\subsection{The ‘Creative Explosion'?}

The Paleolithic art discovered in Western Europe appears rather abruptly in the archaeological record and falls into a relatively small window. During this period the behaviour of the anatomically modern humans who inhabited the area increased in sophistication dramatically, with great improvements in tool manufacture and hunting techniques. These changes have led many archaeologists to argue that a 'creative explosion' took place. But in recent times doubts surrounding this theory have been raised.

Prior to 1994, the oldest known cave paintings - which were located in Western Europe - were no more than 18,000 years of age, but then the Chauvet Cave in France was discovered and its paintings were subsequently carbon-dated to being from approximately 32,500 years ago. This was an incredibly significant archaeological find because it pushed back the age of cave painting by some 14,000 years - yet it occurred less than two decades ago. This relatively recent and highly substantial revision of dates obviously raises the question as to whether there is even older cave art yet to be discovered in Western Europe.

Paintings created in the open-air were almost certainly made (Clottes 2008), but would have long since been destroyed by the elements, so a chance finding of any that have survived is highly 
unlikely. Remnants of engravings have been found out in the open although unfortunately they are extremely difficult to date with any real precision, but as techniques advance, the dating of engravings should become easier. So whilst the chances of finding examples of open-air paintings older than those at Chauvet Cave are small, there is a better chance that older engravings might be found.

Regardless of whether evidence of art produced much earlier in Western Europe emerges, it does seem somewhat counter-intuitive to believe that this phenomenon could have just started suddenly with the arrival of the anatomically modern humans into Europe. Rather than there being a 'Creative Explosion', maybe it was a much more gradual process. It seems plausible to think that these competences evolved slowly and that anatomically modern humans were already producing cruder versions of the art found in western European caves before they arrived; that they carried these skills with them as they moved out of Africa and into Europe.

An important paper published in 2000 by Sally McBrearty \& Alison S. Brooks, ${ }^{7}$ backed up by archaeological data, makes a strong case for such a scenario. They argue that anatomically modern humans living in Africa exhibited a sophisticated behaviour that demonstrates they had developed many important cognitive skills before they arrived in Western Europe. Evidence of improved blade technology, tools made with bone, long distance trading, and specialised hunting techniques, as well as signs of artistic creativity, have all been found.

Steven Mithen (2005) supports this argument by McBrearty \& Brooks and highlights potentially the earliest known representational art from Africa: paintings of bears on portable flat pieces of stone from the Apollo 11 Cave in Namibia. Evidence using comparative dating methods suggests these paintings might have been made 59,000 years ago, but radiocarbon dating indicates a younger age of 28,000 years - although it is possible that this is due to contamination by newer material (Mithen 2005). Ochre has been found in many African sites older than the Apollo 11 Cave (from at least 125,000 years ago) and whilst it has been assumed that it was used for body decoration as no cave art has been found, it's debatable whether this limited evidence can be used to support a claim of symbolic behaviour. However, Mithen (2005) believes that the red pigment used by early Homo sapiens in Africa is much more likely to have had a symbolic purpose than the black pigment used by Neanderthals in Europe, as it has been shown that red is a psychologically important colour for humans. He concludes that as our archaeological knowledge of Africa is severely limited when 
compared to Western Europe, we should be careful of the conclusions we draw about anatomically modern humans in Africa before they migrated to Europe.

Finally, in 2000 a team led by Christopher Henshilwood ${ }^{8}$ found a piece of ochre in Blombos Cave in South Africa: it was marked with several parallel lines in a crosshatch-type fashion and has since been dated to approximately 75,000 years ago (Clottes 2008). It is now generally accepted as being the oldest known piece of art so far discovered, and pushes back the dating of human artistic behaviour by some 35,000 years from the previously earliest examples, i.e. the cave paintings and portable art of Western Europe. Archaeological finds like the incised ochre from Blombos Cave provide further support for the claims made by McBrearty \& Brooks.

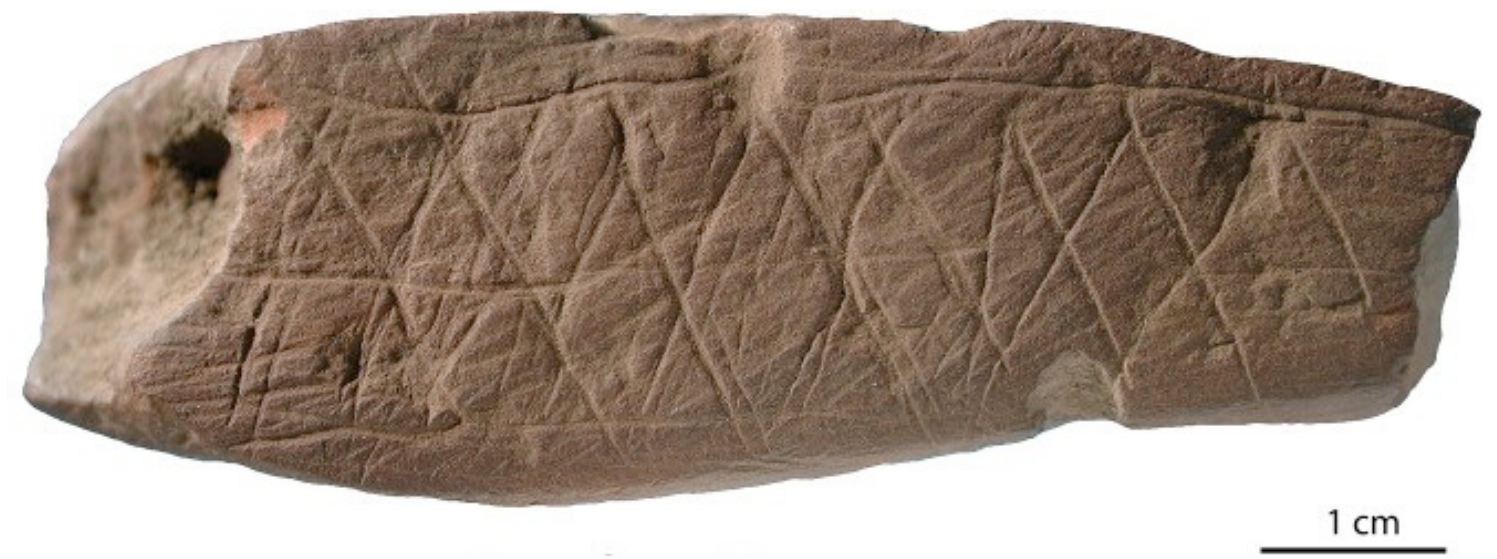

Figure 1.20 Engraved ochre, Blombos Cave, South Africa, approximately 75,000 years old (Henshilwood, d'Errico et al. 2009)

As more discoveries are made, the very least that they will show is that if any 'Creative Explosion' did occur, then it happened much earlier. But if those discoveries produce evidence of gradual improvements in quality and complexity over long periods of time, then this will provide an alternative explanation how our ancestors developed the ability to produce art. One thing that seems certain is that the cave art of Western Europe was built upon African foundations. However, it still remains possible that European art was vastly more elaborate and skilled than its African origins, and that this improvement appeared rapidly, and apparently instantly, upon the arrival of the anatomically modern humans. If this proves to be the case, then further explanation is still required.

\subsection{Did Neanderthals create art?}

It is generally believed that Neanderthals (Homo neanderthalensis) became fully evolved in Western Europe about 200,000 years ago and were descended from the species Homo

8 Dr Christopher Henshilwood has written several papers supporting the claim that the behaviour of modern humans first emerged in Africa, long before its evidence in Western Europe. For his paper discussing the engraved ochre, see (Henshilwood, d'Errico et al. 2002) 
heidelbergensis that had migrated out of Africa and into Europe by around 500,000 years ago (Mithen 2005). Neanderthals existed in Europe up until their extinction approximately 30,000 years ago, some 10,000 years after the arrival of the anatomically modern humans. They had a brain size slightly larger than that of the art producing Cro-Magnons (Guthrie 2005) and it is often wondered whether they too ever created images, but no evidence has been discovered.

It appears that Neanderthals had access to the materials required to create paintings because there are many archaeological finds showing that they made use of pigments such as ochre. Neanderthaloccupied areas dating back to at least 200,000 years ago, as well as burial sites, have been found with pieces of ochre amongst the remnants (Bahn 1998). Furthermore, charcoal from burnt wood would have been a readily available source of drawing material. But no paintings have ever been found that are uncontroversially associated with them (Guthrie 2005).

To further support the claim that Neanderthals did not produce representational art, Steven Mithen (2005) refers to the case of a cave used by them in Bruniquel in southern France, which may also have been the site of their performances such as singing and dancing. Jean Clottes (reported in Mithen 2005) highlights the striking fact that there is no Neanderthal cave art there, despite apparently perfect conditions for creating it.

Although no Neanderthal representational art has been discovered, they do appear to have undertaken symbolic acts (a cognitive requirement for producing art) including selecting aesthetically appealing stones for tool-making (Guthrie 2005). There is also evidence that Neanderthals buried their dead (McBrearty and Brooks 2000), which could be interpreted as a symbolic act, but it may also have been for the purpose of either creating a more hygienic environment or of deterring scavenging animals (Guthrie 2005). Neanderthals also made jewellery, and recent archaeological evidence dates the earliest so far known in Western Europe to as far back as 50,000 years ago (Zilhão, Angelucci et al. 2010).

Mithen (2005) speculates that although Neanderthal brains were undoubtedly large, they only had a 'domain specific' capability - each specialised area was very much isolated from the others. This enabled them to be very good at individual tasks such as tool making, but restricted their brain's ability to produce creative thought due to a lack of 'cognitive fluidity'. Mithen goes on to say, "I suspect that Neanderthals would have exploited ... the shadows thrown up against the cave walls by the firelight, to make their singing and dancing more dramatic" (Mithen 2005, p. 243). But he 
argues that when Cro-Magnons also saw these shadows, they instead were inspired to draw the images of the animals that they conjured up.

So despite a significant brain size and access to the necessary materials, Neanderthals do not appear to have created any cave art and this type of behaviour only began in Western Europe following the arrival of the anatomically modern humans. They may however have participated in body painting. And if as Mithen claims, Neanderthals danced and sung then some would interpret these acts however basic - as being a form of art. Thus, although Neanderthals might have participated in some forms of artistic behaviour, they did not produce physical representations; they did not depict.

\subsection{What wasn't drawn}

Only about twenty complete drawings of humans have been found amongst all the Paleolithic cave art of Western Europe (Clottes 2008). And none of those are in any great detail. In contrast, their naturalistic drawings of animals clearly demonstrated great artistic skill. Many of the incomplete images that they made allude to minds obsessed with thoughts of a sexual nature: drawings of vulvas and the occasional erect penises. Handprints are in abundance on cave walls and provide the only truly accurate representations of any part of the human body.

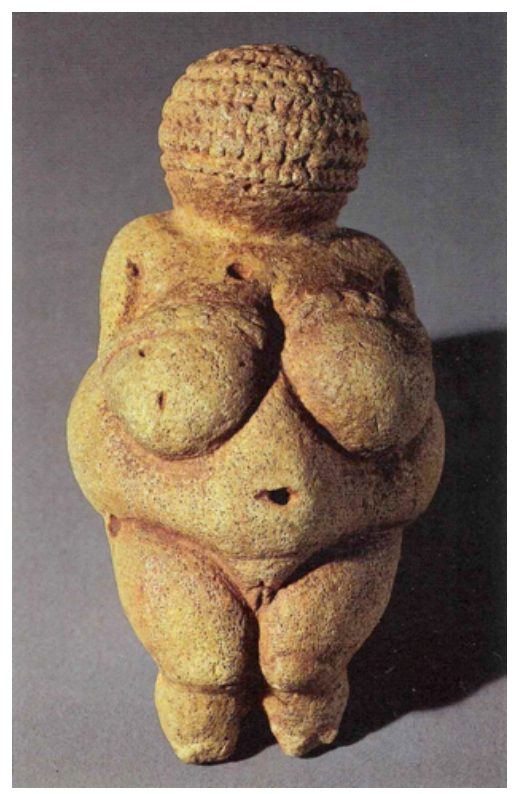

Figure 1.21 Venus of Willendorf statuette, recovered from a site in Willendorf, Austria 22-24,000 years old, approx 11cm in height (Spivey 2005)

The most detailed representations of a complete human body are the carvings known as the Venus figurines, but they are not naturalistic in appearance and their exaggerated breasts and buttocks 
suggest a strong sexual influence. Importantly - and I think highly significantly - none of these figurines contain any sort of facial detail - apart from one. This solitary figurine, named the Venus of Brassempouy (after the location in France where it was found), does have a detailed face and has been dated to approximately 25,000 years ago - but its finding has been extremely controversial because the workers at the excavation site were rewarded for their finds (Bahn 1998). Even more striking, is the fact that another of these figurines did have its face carved rather than just left smooth, but the Venus of Willendorf's face (and the rest of its head) is just a pattern of raised dots (see photo above) - its maker clearly chose to address the details of the face, yet decided to deliberately replace them with featureless marks. The dots have been suggested as representing braided hair (Spivey 2005). The detail of the Venus figurines often include even nipples, so any claim that they contained only a minimal level of detail can be dismissed. Yet they do not even possess a basic nose, mouth, and two eyes. Ruspoli (1987) speculates that the lack of facial detail may have a religious explanation, but admits that we will never be able to understand the mentality of Cro-Magnons as it would have been so different from that of today.

Another form of representation is that of the anthropomorph: an entity that is half-human and halfanimal. One of these is the 'Lion-Man of Hohlenstein' figurine where the top half is the carving of a lion, whilst the bottom half is that of a man's body. There is also a painting of 'The Sorcerer' that was found at The Sanctuary Cavern at Trois-Frères, Ariège, France: the top half is a drawing of a Stag, and the lower part is that of a man. Interpretations of these anthropomorphs are that they were of a religious nature, possibly linked to shamanistic rituals. The work done by Jean Clottes and David Lewis-Williams in this area is discussed in detail in the next chapter. 

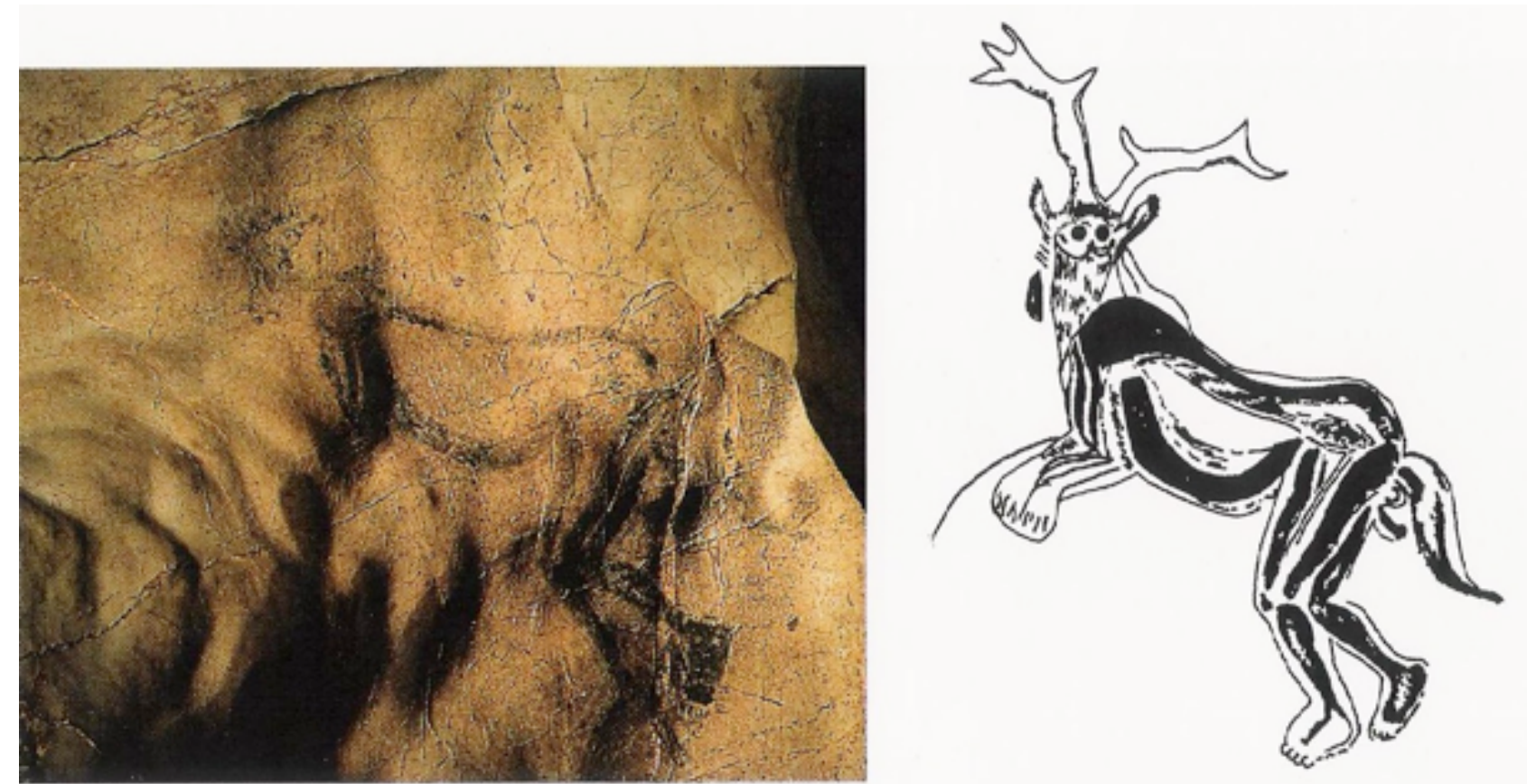

Figure 1.22 (above, left) Composite being 'The Sorcerer', found in the Cave of the Trois-Frères, approximately 16,000 years old (Bahn 1998;

Clottes and Lewis-Williams 1998)

Figure 1.23 (above, right) Henri Breuil's sketch of 'The Sorcerer' (Clottes and Lewis-Williams 1998)

The almost total lack of any sort of narrative is also perplexing (Guthrie 2005). The whole community's lives were dependent on the success of hunts, yet despite the importance of hunters, as well as the complete hunting experience, there were only limited depictions of either. Along with a general lack of human representation, it might have been expected that the artists would have drawn close relatives, their children, or even their partners; the fellow humans who were important to them. No drawings have been found containing landscapes, smaller animals, insects, plants, the sun, the moon, or the stars (Clottes 2008).

This section raises some interesting and important questions that are worthy of future research. Some of the issues above, such as the sexual, mystical and religious themes of cave art, are addressed by the theories discussed in the following chapter, but what wasn't drawn is just as important as what was. Attempting to discover the reasons why the artists chose not to draw certain subjects might provide further insight into their motivations for creating the drawings in the first place. 


\subsection{Why didn't Paleolithic art happen earlier?}

The arrival of Homo heidelbergensis (the last common ancestor of Homo sapiens and Homo neanderthalensis) approximately 500,000 years ago appears to be a significant development. Its brain size was considerably larger than its predecessors and close to that of modern man. Archaeological discoveries have shown that they were also capable of producing much more complex and finely crafted tools (Balter 2009). Based upon the fossil evidence, it has also been suggested that they had capacity for speech (Mithen 1998). Unfortunately, due to the lack of preservation we will probably never know whether they produced art, but there are hints of an emerging artistic tendency. As discussed previously, the much more symmetrical tools being produced may be signifying an increasing appreciation of aesthetics. And if some of their handaxes were used for attracting mates, then their possession of an ability to appreciate symbolic meaning would indicate the presence of an additional key requirement for producing art. I discuss the connection between symbolic thought and art in chapter 3 .

The issue of taphonomy is an important factor when considering whether earlier examples of art ever existed. Most Paleolithic art would not have survived due to decay and exposure to the elements, and what has survived is certainly a highly distorted sample (Bahn 1998; Guthrie 2005). The older an object, the greater the chance it would have of being destroyed. Also, as we look further back in time only routine material culture will be preserved, whilst rarely made items will be less and less visible. Arts such as dance and storytelling, which would demonstrate a growing creativity, leave no trace in the archaeological record.

Due to the sheer quality, it seems highly likely that cave art was preceded by drawing on nondurable surfaces such as the open ground, in sand, mud, or scratches on wood, but it is doubtful that there will ever be any proof of this. R. Dale Guthrie (2005) references the work of Ann Sieveking, and states that there is plenty of evidence to suggest that caves were not often frequented. So it is probable that most art was created outside of the caves and thus the art found in them represents just a small sample of the overall total that once existed outside.

The discovery of caves like Blombos in southern Africa that contain engraved objects and jewellery, show that some forms of art were being practised at least 75,000 years ago. Whilst sites such as the one at Qafzeh in Israel from 100,000 years ago, plus the many older sites that have also produced lumps of ochre, all provide hints of artistic behaviour. It therefore seems likely that the advent of cave painting and engraving were the later stages of a series of gradual developments. 
Earlier expressions of a growing ability to produce art (and indications of an increasingly creative mind) have been mostly lost to the ravages of time, but there are some artefacts that provide us with tantalising glimpses of what was happening.

\section{CHAPTER SUMMARY}

Paleolithic cave art occurred for a period of approximately 25,000 years and ceased at the end of the most recent Ice Age, some 10,000 years ago. The end of this Ice Age produced major environmental changes leading to enforced upheavals for the anatomically modern humans who lived in Western Europe. Sea levels dramatically rose, altering coastlines and submerging once inhabitable areas. Glaciers melted and previously uninhabitable regions came to life, offering new hunting grounds. Forests spread into previously open spaces, and animals such as deer that formed a major part of their diet, moved into new areas of lush vegetation that had previously remained baron - and some Paleolithic people moved with them. The invention of farming and the change to a sedentary life followed. The caves that were used to produce art over many thousands of years were left deserted and forgotten - until they were found again a little over one hundred years ago (Ruspoli 1987).

The act of creating cave art was a particular type of behaviour that persisted through the generations, as there are too many similarities for it to be an isolated set of spontaneous events (Clottes 2008). However, interpretations of many features of cave art - including those that are outside of the norm - can easily be manipulated to suit a certain theory, for much of the theory of cave art is extremely subjective. The discovery in Europe of just 350 caves so far, when the period of production spanned some 25,000 years, suggests that there are either many more caves yet to be discovered, or that most examples have now disappeared. And due to this relatively small sample, it makes it highly likely that any new discoveries may well produce new revelations that challenge our current thinking about this phenomenon.

The collection of the pigments needed for cave painting required well planned out expeditions and their preparation and subsequent use involved a considerable amount effort. Although it is possible that when cave painting began, the pigments for drawing on the cave walls (like the charcoal and ochre) were readily available from being used for other purposes. The fat used in the lamps needed to be extracted from the hunted animals, which would have also involved an additional cost. Then 
there was the amount of time required for each venture that might have been better spent on more life-sustaining activities. Even the energy exerted in roaming the cave complexes could have been directed towards hunting food instead. For the deeper cave incursions, the time, effort, and material costs would have been amplified. And venturing into the caves to make the art introduced unnecessary dangers. All of the above suggests that the motivation to produce cave art must have been strong.

Whilst the identities of the Paleolithic cave artists remains unknown, there has been much speculation surrounding them. Were they just a small percentage of the population, or a larger proportion? And if the former, then was the creation of cave art restricted to a privileged few, or just those with the greatest technical ability? Issues concerning their sex, age, demographic background, and whether they had anything in common with each other are also important questions. Attempting to determine if cave art was the result of a culturally reinforced behaviour, or the product of a few random individuals, could lead to a better understanding of how and why this phenomenon occurred and what the motivations were for this type of behaviour.

In the next chapter I intend to examine some of the early theories that have since been rejected, plus a deeper analysis of some of the more important contemporary ideas that have been put forward. The style, choice of subject, location, and chronology of the Paleolithic cave paintings have all been analysed at length, with resulting hypotheses put forward offering suitable explanations about the production of this incredible example of early art. Due to the very nature of the drawings however, many facts about their creation will never be known and thus attempting to analyse the motivation of these people will always remain to a large extent, beyond reach.

There have been many other forms of art created from the Paleolithic era onwards, and it is clear that right from the start of this unique human behaviour people all over the world were beginning to express themselves and to leave their mark on the their environment. The subject matter of cave paintings provides us with some insight into what was important to Cro-Magnons and what was on their minds. Similarly, questions can also be raised concerning what was not drawn on these cave walls. All in all, the production of cave paintings required a considerable amount of time and effort - something that has important implications for what I will be discussing in the next chapter. 


\section{Chapter 2: Evolutionary Issues}

\section{INTRODUCTION}

Producing art incurs many costs, so what was there to gain from participating in this type of behaviour? In this chapter I will be addressing the issue of what motivated our ancestors from the Paleolithic to create cave art in particular, as well as art in general. I will summarise and then assess some of the main theories that have so far attempted to explain the questions surrounding artistic behaviour.

I begin the chapter by explaining how evolution can account for changes in an organism's physical structure or its behaviour. I then briefly provide an overview of the two main types of explanation and the main principles involved. I next move on to discuss some of the important initial theories that were proposed, before taking a longer look at several contemporary theories that argue either for or against there being an evolutionary advantage for artistic behaviour. I will then summarise what has been discovered and what further analysis is possible.

\section{WHY DRAW?}

The discovery of cave art over one hundred years ago raised many questions. The initial focus was towards the paintings, with attempts being made to search for chronological styles and whether there was any significance in their location within the caves (Clottes 2008). Later on the attention turned towards the artists, with questions being asked of their motivations for creating the paintings and what (if any) meaning they might have attached to them. Addressing the issue of motivation raises important evolutionary issues when searching for the possible causes that produced this type of behaviour.

When examining the motivations behind the artistic behaviour of hominids during the Paleolithic, there are two concepts to consider. The first, evolutionary biology, concerns how our ancestors have changed over generations, and what possible selective pressures have led to those changes. The second, evolutionary psychology, addresses how it may be that past changes affect the behaviour of 
the present. I will now briefly discuss these two approaches and how they relate to the issue of art making.

\subsection{Evolutionary Biology}

Charles Darwin's theory of natural selection predicts that an organism's physical form and behaviour will change over time in response to the varying environment that it encounters. There are three requirements of an organism for this to occur. Firstly, there has to be variation between individuals within that organism's population; secondly, an individual's offspring must be able to inherit this variation; and thirdly, these variations need to affect each individual organism's chances of survival and its ability to reproduce within its environment - this is generally referred to as its 'fitness' (Boyd 2009).

There are three possible ways that a change can occur to an organism as it evolves. The first is called an adaptation, which is a permanent physical or behavioural change in an organism and its offspring brought about by environmental pressures; the result is an improved fitness of all those individuals that inherit this change (also referred to as a 'trait'). The second change is called a byproduct, because it is a side-effect of an adaptation and not the direct result of a change that improved an organism's fitness. A third possible change is due to a random one-off mutation that may or may not improve an organism's chances of survival or reproduction (Dutton 2009).

Changes within a population are also subject to an element of chance, termed 'genetic drift'. Genetic drift occurs when some individuals, just by chance, leave behind more descendants than others - possibly due to a natural disaster like a volcanic eruption, or some other random event. A consequence of these events is that they produce a bias within a population based upon luck, rather than how well adapted to their environment particular individuals may be. Genetic drift has more of an impact on small populations.

Adaptations occur gradually over many generations, and whilst they improve the organism's chances of survival or reproduction at the time of their occurrence, they may or may not have a beneficial effect for later generations. An example of this is the human appendix: it evolved to assist in the digestive process, but due to a subsequent change in diet its original function (possibly for digesting leaves) is now redundant - but it still has (unwelcome) effects on our bodies, such as appendicitis. 
An evolutionary by-product (also referred to as a 'spandrel') may have an effect on an organism's fitness, or it may be neutral. A regularly used example of an evolutionary by-product is the whiteness of bone, which is caused by the calcium that it contains (Dutton 2009). But what matters about a bone is how strong it is (its function), not its colour. If calcium were a different colour then bones would be that colour too. In the case of this by-product, the colour of bone has no impact on either a vertebrate's chances of survival, or its reproductive success.

The appearance of art and culture in human society does not appear to sit well with the standard view of Darwinian evolutionary theory - art requires a lot of time and effort, yet it is not immediately obvious how it improves fitness. If artistic behaviour is an adaptation, then it must be shown how it either enhances the chances of survival or improves reproductive success. This obviously has implications for the production of cave art and its origins, as well as art in general.

Theories that attempt to explain the phenomenon of cave art must also be able to provide an evolutionary story of how and why anatomically modern humans began this particular type of behaviour. There is a question of whether cave art was produced as the result of a genuine adaptation, or if it was the outcome of an evolutionary by-product. As Dutton points out, the possibility of it being caused by a random one-off mutation is ruled out due to humans having been persistently involved in art-making activities over an extended period of time (Dutton 2009). Likewise, the cost of art suggests that it is not a neutral change, and so not the result of genetic drift.

\subsection{Evolutionary Psychology}

Evolutionary psychology is a sub-field of evolutionary biology and involves the study of the brain with regard to its evolution and function. Any adaptations within the brain must also conform to the requirement that they either increased the chances of survival or reproduction - or both. Evolutionary psychology makes observations of current behaviour and then attempts to explain those actions based upon what is known of past influences and the adaptations in the brain that they have produced.

An animal's behaviour changes as it adjusts to its environment; over the course of an organism's evolutionary history, behaviours that improve its fitness will become innate in later generations in the form of psychological adaptations. A regularly used example of this is a monkey's fear of snakes, and how even those raised in captivity - and having never encountered a one before - still react by attempting to immediately move away from a snake (Pinker 1997). 
But some innate behaviours can be triggered by other means: a piece of plastic tubing that roughly resembles a snake will produce the same response from a monkey as would a real snake (Pinker 1997). Evolved behaviours can affect an animal's response when encountering a new experience. Distinguishing between the historical and current causes for a particular behaviour is important when attempting to explain that behaviour. Explaining an animal's behaviour by referring to its evolutionary history is what is termed its ultimate cause, whilst what stimuli have triggered the current response is termed the proximate cause.

In humans, a good example is our desire for sugary and fatty foods. During our evolution, sugary and fatty foods provided an essential requirement for our survival, and so our ancestors developed the psychological adaptation to like any food that contained these tastes - this is the ultimate cause of our liking of sugary and fatty foods. But we now know that too much of these foods are bad for our health, yet we still crave these flavours in modern foods such as chocolates and hamburgers; the taste of these foods are the proximate cause of our liking them (Dutton 2009).

So the reasons for creating art may have been psychological rather than caused by selection pressures. Artistic behaviour may be motivated by any number of stimuli that are ultimately connected to ancient adaptations that had absolutely nothing to do with art. The mental states of the artists may have, for instance, resulted in them wishing to banish fears, or cater to superstitions. This same possibility also applies to the creation of cave art.

An example of this proximate/ultimate explanation of an animal's behaviour can be given from the action of creating cave art. It has been previously noted that many of the Paleolithic cave paintings made use of suggestive protrusions in the cave walls. The shadows projected by the cave contours may have produced shapes that caused those who saw them to believe that they were seeing certain animals. These shadows are the proximate cause of seeing imagined animals. The ultimate cause of this is the evolved adaptation to detect shapes and patterns.

\subsection{Summary}

The question I will address in the remainder of this chapter is whether cave art was a spandrel some kind of by-product of our ancestors' evolution - or a true biological adaptation? In the following sections I summarise and evaluate the major theories that have been put forward concerning the origins of cave art as well as the origins of art in general, including some early 
theories that have now been dismissed. Each theory attempts to explain the evolution of artistic behaviour, with some theories specifically addressing the phenomenon of cave art. Some of the theories are also able to explain why we continue to participate in this activity, whilst others only provide ideas concerning why it first started.

\section{Early theories}

Once the first examples of cave art were discovered, theories began to emerge that attempted to explain the phenomenon. Most of the early theories were quickly dismissed, but during the middle of the last century two theories in particular were taken seriously for some time. They provide a useful background to the modern theories that I will discuss later on in this chapter, and I will shortly discuss them below. But firstly I will explain both the context in which the first discoveries of cave art were made, and also provide a brief history of the earliest thoughts on the subject.

When appraising the earliest theories of cave art, it is important to realise that they were all based upon the belief that the Cro-Magnons who created it possessed only a very primitive mind. This presumption resulted in the automatic exclusion of any theory that required the artists give significant deliberation to the act of creating a painting. Thus, the simplest explanation was to argue that cave art was nothing more than a form of elaborate decoration, a product of an increasing amount of available time (Lewis-Williams 2002). Indeed, one would have been ridiculed for suggesting that these Paleolithic people were anything other than 'dumb brutes'; the harsh treatment dealt out to Marcelino Sanz de Sautuola who discovered the cave art of Altamira, bears testament to how anyone who went against the dominant line of thought was treated. A complex reason for producing a piece of cave art would have required the possession of a complex mind.

However, the discovery of cave art in remote and inaccessible areas of the caves began to throw doubt upon the claim that it was merely for decorative purposes (Lewis-Williams 2002). Anthropologists became involved and ethnographic comparisons began to be made with existing hunter-gatherer groups; the possibility that Paleolithic people had some early religious tendencies started to be considered. During this period the common belief was that all the images of cave art had symbolic overtones and anyone studying the subject would always be attempting to identify an underlying theme (Guthrie 2005). For some, the apparently sudden desire and ability to create these drawings was so different from any other previous behaviour of anatomically modern humans that 
it seemed to be a reasonable assumption that it was driven by newly acquired religious thoughts or magical beliefs (Guthrie 2005).

Whilst these early theories attempted to explain the motivations behind cave art, they did not directly address the evolutionary issues of adaptation and by-product. As these theories have now been dismissed, I will not be analysing them from an evolutionary standpoint and will instead present them solely from a historical perspective. I will now describe in more detail two of the most popular early theories that attempted to explain the origins of cave art.

\subsection{Abbé Henri Breuil: 'Hunting Magic'}

One of the earliest experts in the study of cave art was the French Catholic priest Abbé Henri Breuil, who spent most of his life investigating this phenomenon - so much so that he was given the nickname the 'Pope of Prehistory' (Clottes 2008). In the early part of the last century he believed that the paintings followed a pattern that highlighted a chronological trend, and he also speculated what they might have meant to the artists who had drawn them.

His first theory claimed that cave art had developed in two consecutive and distinct cycles, each time starting out as rather crude attempts, but then gradually improving in artistic skill and complexity of representation. But this idea was subsequently shown to be inconsistent (Bahn 1998). For example, he regarded some drawings as having a primitive style known as a 'twisted perspective' (where some parts of the animal's body such as its horns were drawn skewed from the rest of its body) - yet this style was seen repeatedly over different cultural periods (Chauvet, Brunel Deschamps et al. 1996).

The many images of large herbivores led Breuil to believe that the artists were obsessed with these animals for some reason and prompted an additional theory. After examining many of the images, he claimed that they were often marked with small lines that he believed were representing the spears used to hunt the animals (Lewis-Williams 2002). He therefore argued that the paintings were 'hunting magic' that would provide the group with success in future hunts by gaining some kind of magic control over them (Guthrie 2005). 


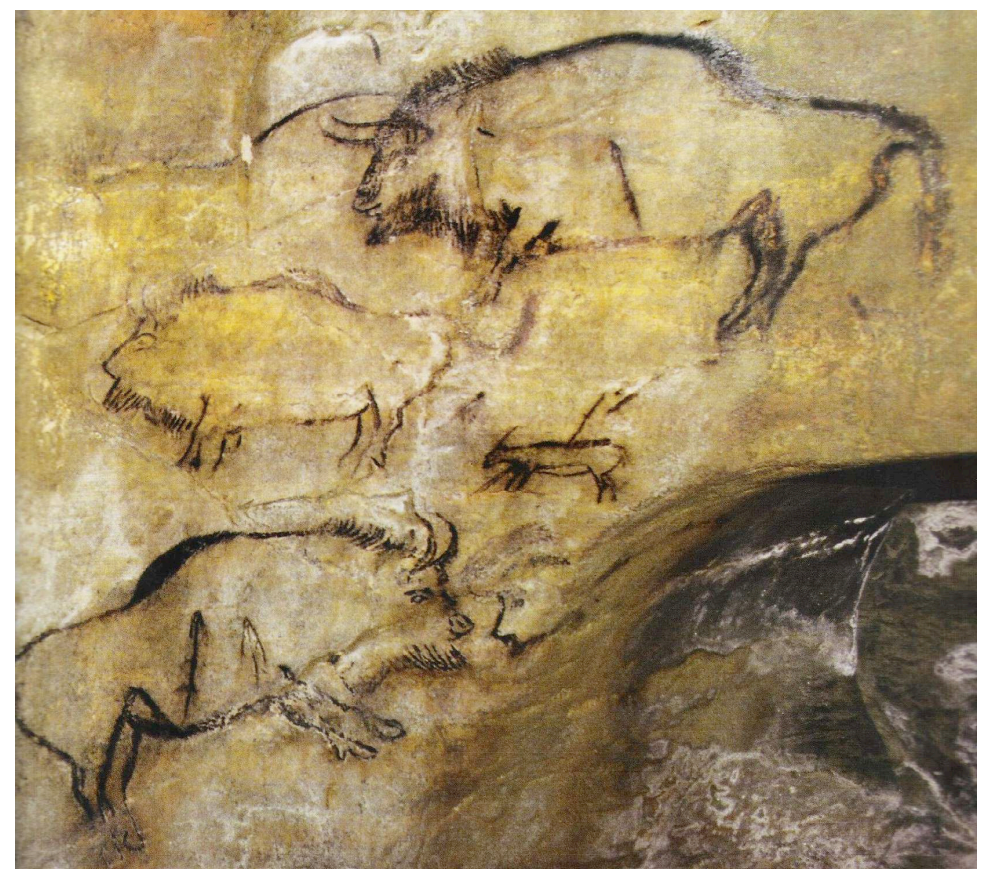

Figure 2.1 Bison and Ibex approximately 13,000 years old (Upper Magdalenian), Niaux Cave, Ariège, France (Clottes and Lewis-Williams 1998; Clottes 2008)

Breuil also argued that the many images of a sexual nature were for the purpose of fertility magic to improve the fertility of the females in the group. He supported his interpretations of hunting and fertility magic with the use of selected ethnographic analogies (Bahn 1998), whereby modern-day hunter-gatherers are observed and then inferences are made about how Paleolithic people lived some 40,000 years ago.

Whilst Breuil's latter theory was accepted for a while (it seemed plausible that cave art was a kind of pre-historic witchcraft that was also linked with hunting (Lewis-Williams 2002)), cracks gradually began to appear in his deductions and the theory was eventually discredited. The animals painted in the caves did not reflect local diets (based upon analysis of food remains), apparently contradicting the 'hunting magic' claim (Spivey 2005) - although it's possible that the drawings were of animals that they wanted. The discovery of drawings of predators (such as lions) alongside those of prey required further, less plausible, explanation. Breuil subsequently argued that these images must have been drawn to bestow the powers of these dangerous beasts onto the hunters who drew them (Lewis-Williams 2002). But then to further weaken the theory's plausibility it was claimed that some of the increasingly prominent abstract markings represented animal traps; other highly tenuous ad hoc explanations continued to be made as and when required (Lewis-Williams 2002). Gradually, as more caves containing art were discovered, the theory became less explanatory and increasingly incredible. The theory was not testable and explanations could always be tailored 
to fit the latest finding (however far-fetched they seemed to be). Eventually a new theory emerged that appeared to provide a better explanation of cave art's origin and motivation.

\subsection{André Leroi-Gourhan's structuralism}

In the 1950's and 1960's French archaeologist André Leroi-Gourhan, along with his student Annette Laming-Emperaire, introduced their theory of cave art. They argued that the artists who created the cave images were much more intelligent than had been previously thought. Their research led them to believe that Paleolithic people were intensely superstitious and the images they created had symbolic meaning. They thought that the images had been deliberately placed in specific locations within the caves to produce a planned composition that was part of a symbolic system, rather than being placed randomly as had previously been assumed (Lewis-Williams 2002). Unlike Breuil who used ethnography to supports his claims, Leroi-Gourhan and Laming-Emperaire rejected any use this potentially error-prone method of analogy (Lewis-Williams 2002).

They began their investigations by rigorously detailing the type and location of Upper Paleolithic images found in the caves of Western Europe. They believed that the images were drawn in pairs and assigned the category of 'maleness' or 'femaleness' to specific groups of animal images. Additionally, apart from the more obvious drawings of human genitalia, they assigned the more cryptic signs as representing male or female genitalia based upon how narrow or wide they were (Bahn 1998).

The location of each image was carefully noted and placed into respective groups or 'schemes' (Ruspoli 1987). They then argued that 'male' images were spread around throughout the cave complexes, but that 'female' images resided solely in the central chambers of the caves (LewisWilliams 2002). This then led to them declaring, for example, that horses and deer represented males, whilst bison and aurochs represented females. To apply some kind of chronological structure, four different styles were discerned (unlike the two that Breuil had previously detailed), with each one then being deemed to belong to a particular period of cave art's history (Ruspoli 1987). The theory became known as structuralism.

A central claim of structuralism was that whilst the images became more complex over time, there were no dramatic changes in style. However, the discovery of Chauvet cave with its incredibly complex drawings from up to 33,000 years ago, contradicted the idea of gradual improvement in complexity (Lewis-Williams 2002). Leroi-Gourhan had claimed that the most commonly depicted 
animals (bovines and horses) were grouped together in the central area of cave paintings and the rarer animals were drawn in the periphery, but once again, the discovery of Chauvet cave contradicted this with felines being in mostly dominant positions (Chauvet, Brunel Deschamps et al. 1996).

As more images were discovered and the technique of radio-carbon dating was introduced, it became clear that the chronological styles that had been attributed to the various images were unreliable (Bahn 1998). Also, as more cave systems were discovered, many were found to be lacking a central chamber, one of the main requirements of the theory. In the end, the evidence did not support the theory.

\subsection{Summary}

Regardless of the accuracy of their theories, the research and subsequent cataloguing of cave art's many images carried out by Leroi-Gourhan, Laming-Emperaire, and Breuil has provided more contemporary researchers with a wealth of accurate data to base their work upon. Breuil argued that the images were the result of magical beliefs, and Leroi-Gourhan and Laming-Emperaire shifted the focus to their having a symbolic meaning, but in the end neither theory was able to fully explain the increasing diversity of images and cave formations that were being discovered. They were not precise and rigorous enough to be testable.

Guthrie believes that two factors hindered the study of cave art during this initial period. Firstly, there was a strong prejudice that because the Paleolithic artists led primitive lifestyles, they therefore possessed limited cognitive ability. And secondly, there was also a preference for drawing dangerous ethnological parallels. Together, these assumptions introduced many errors that stifled much of the progress of the early investigators (Guthrie 2005). It also appears that the first theories were quite selective in which drawings they used to support their theories; if a particular drawing didn't fit, then it was ignored (Guthrie 2005).

So whilst early cave art theories were flawed, they did, however, provide some useful foundations for subsequent research. I now wish to discuss several of the more prominent theories currently being debated that attempt to explain either art in general, or in some cases cave art in particular. These contemporary theories take an evolutionary view, including whether it was the result of an adaptation or a by-product. 


\section{ART AS A BY-PRODUCT}

According to the evolutionary psychologist Steven Pinker, activities such as cave art were a purely frivolous development with no obvious benefits. As he provocatively puts it, participating in the arts is nothing more than "Sunday afternoon projects of dubious adaptive value" (Pinker 1997, p. 524). Cave art, according to this view, was a by-product of our evolution; it serves neither a reproductive, nor a survival purpose, and is therefore not evolutionary adaptive. Producing art in this way, with no ulterior purpose other than creating individual gratification, is known as 'art for art's sake'.

Pinker has described this type of behaviour as a kind of pleasant treat for the mind and in particular he famously refers to music as being merely "auditory cheesecake" (Pinker 1997, p. 534). The motivation for humans to produce art, he argues, is simply because it pushes all our 'pleasure buttons', in very much the same way that we today have a preference for eating of sugary and fatty foods (as previously mentioned). As such, it is a throwback to our evolutionary past in that it indulges the desires associated with ancient adaptations. Pinker claims that creating art (the proximate cause) provides us - and previously our ancestors - with a way of catering to our evolved aesthetic preferences (the ultimate cause) whilst at the same time it also appeals to our psychology of status within a group (Pinker 1997).

But as we saw in chapter 1, the cost of making cave paintings was high. Furthermore, the creation of cave art in Western Europe lasted for perhaps 25,000 years. For this type of behaviour to continue for so long, given the costs involved, there would have been real selection pressure against it if it had no fitness advantage. Any variation that produced individuals with a lack of interest in artistic behaviour would see them being favoured.

I will now describe and then appraise two theories of cave art that point towards it being an evolutionary by-product.

\subsection{Shamanism}

\subsubsection{David Lewis-Williams and Jean Clottes}

One of the most popular and well-known theories about cave art is advocated by David LewisWilliams. In his 2002 book 'The Mind in the Cave', Lewis-Williams argues that shamans created 
cave art after experiencing hallucinations during rituals. French archaeologist Jean Clottes, one of the world's experts in the field, firmly believes that this theory is the best explanation for the cave art of the Upper Paleolithic based upon our current knowledge (Clottes 2008). To further support the argument, Clottes points out that the evidence suggests that only a few people were involved in the production of the art (Clottes 2003). Clottes has co-authored several books with LewisWilliams.

According to Lewis-Williams, altered states of consciousness have been almost completely ignored by archaeologists during their various research, and they have made the presumption that all ancient behaviour was the product of a rational and clear-thinking mind (Lewis-Williams 2002). He questions this approach when investigating the phenomenon of cave art. Anyone spending prolonged periods of time in dark caves would, he believes, have been cut off from almost all their sensory stimuli, leading to the possibility of them having hallucinations (Clottes 2008).

Quoting research into sensory deprivation, Lewis-Williams argues that as the hallucinations it causes proceed through their different stages, they produce visions that reflect what images have been found on cave walls, including geometric forms, animals, therianthropes (transforming human-animal figures), and sexual organs (Lewis-Williams 2002). Furthermore, he claims that the modern humans of the Upper Paleolithic were anatomically like us, so their brains would have been capable of similar experiences (Lewis-Williams 2002). Induced trance can produce these same visions.

To support his argument, Lewis-Williams relies heavily on ethnographic comparisons with several modern-day hunter-gather tribes, in particular the San people of Southern Africa and their shamanic practice of self-induced trance. Until approximately one hundred years ago when the San were displaced from rocky terrain and forced to the desert, their shamans produced a large amount of high quality rock art (Spivey 2005). They made paintings of their visions and told stories of how they had been in touch with the spirit world during their trances, which they claimed gave them the power to cure the ills of the sick (Lewis-Williams 2002). Just like the art-producing shamans of the San, Lewis-Williams believes that Paleolithic shamans also created it, in caves. 

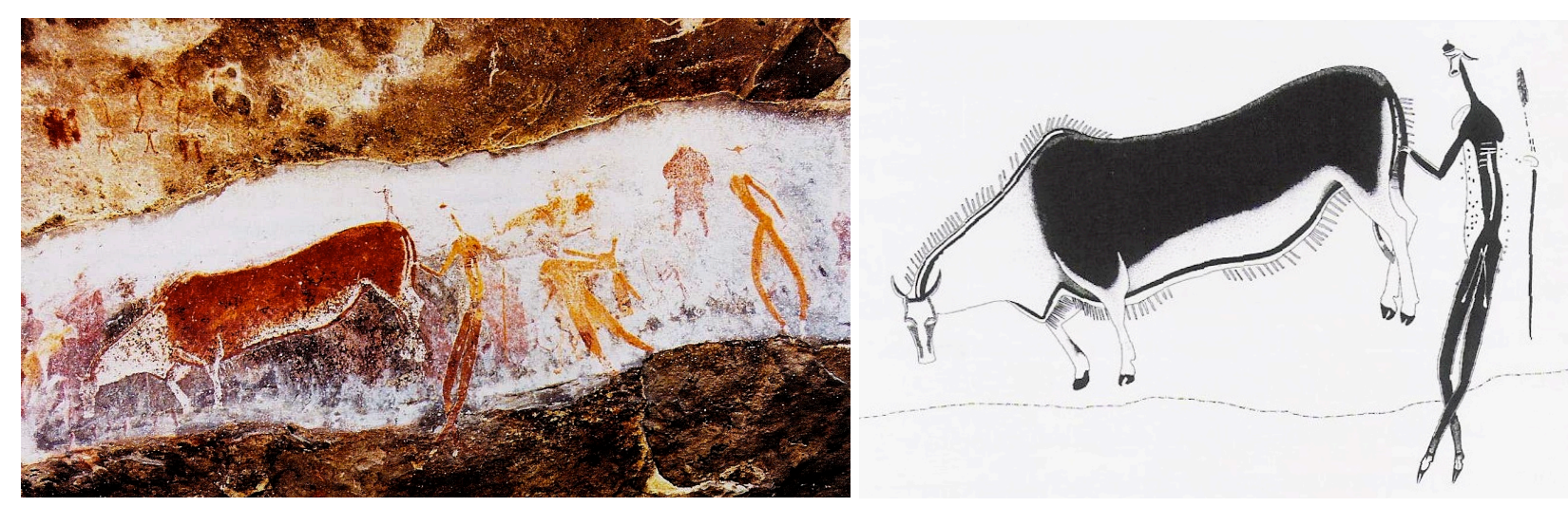

Figure 2.2 San rock painting of hybrid human-animal figures (therianthropes) and an eland (type of antelope) (Spivey 2005)

Lewis-Williams also provides a hypothesis for how the act of image making first started. He claims that whilst experiencing their hallucinations, Paleolithic people reached out to 'touch' the visions that would seem to be on the cave wall's surface. They would trace the visions out, and if this happened on soft surfaces then an impression was left after the vision had disappeared (LewisWilliams 2002). Jean Clottes also believes that the evidence suggests that the cave walls, ceilings, and floors appear to be significant to the Paleolithic people and that they seemed to be trying to penetrate the surfaces to reach a spiritual world beyond them (Clottes and Lewis-Williams 1998). Clay cave walls pierced by spears and fingers, a variety of bone objects (some engraved) thrust vertically into cave floors, and pieces of bone and quartz deliberately placed in small cracks and fissures, all appear to support this claim (Clottes and Lewis-Williams 1998)

Thus, the mental images were the result of a kind of quirk in the nervous system, they were not caused by any evolutionary cognitive development or adaptation, and they were not representations. To support his claim, he points out that Upper Paleolithic cave paintings float freely in space with no reference to their natural environment - exactly like visions. In the final part of this process, other people who saw the completed paintings, but had not experienced the visions themselves, began duplicating them (Lewis-Williams 2002).

\subsubsection{Analysis}

From an evolutionary standpoint, this theory provides a hybrid explanation of how cave art originated. It argues that shamans entered caves with painting equipment, fell into a trance, and then painted what they saw during their hallucinations. The ability to enter a trance was made possible by a quirk in a nervous system that had evolved over many adaptations, over millions of years, to continually improve its owner's fitness. When the information it is primed to receive is restricted, a 
combination of those neurological adaptations is capable of producing hallucinations as an evolved by-product. Spending a period of time in the total darkness of a cave, which also limits the inputs to other senses, triggers such a scenario. Observations of modern-day shamans show that trances can also be hastened, or even self-induced, by heightening psychological states with the use of chants and rhythmic body movements.

The rituals associated with shamanistic practices would also have improved sociality by bringing the group together, and it would have instilled a social order with the shamans gaining a leadership role. This method of promoting group bonding and social structure would have improved overall cooperation, an adaptive advantage. Additionally, the superstitious (and possibly religious) thoughts associated with shamanic practices may also have been a by-product. An increasingly imaginative mind, which would be adaptively advantageous for its improved problem-solving abilities, may also have been capable of producing these irrational beliefs. Shamanic rituals may have been a way of focusing these thoughts and generating positive benefits.

Whilst this shamanistic theory seems somewhat far-fetched and relies heavily on ethnographic comparisons that can add further doubt (Guthrie 2005), it has the support of many Paleolithic art scholars including Jean Clottes and so deserves to be mentioned here. It does, however, lack a complete explanation of all of cave art - for instance, not all cave art was created in locations that could have been used for rituals (e.g. deep underground in very small, hard to access chambers). Also, some paintings were made by a number of people who cooperated together (Clottes and Lewis-Williams 1998). Despite this, there are many indications that seem to show Cro-Magnons were deeply superstitious, such as the deliberate placing of a cave bear skull in the middle of a block of fallen stone in Chauvet cave (as mentioned in chapter 1) lays testament to this; pieces of bone and quartz wedged into cracks are another (Clottes 2003) 

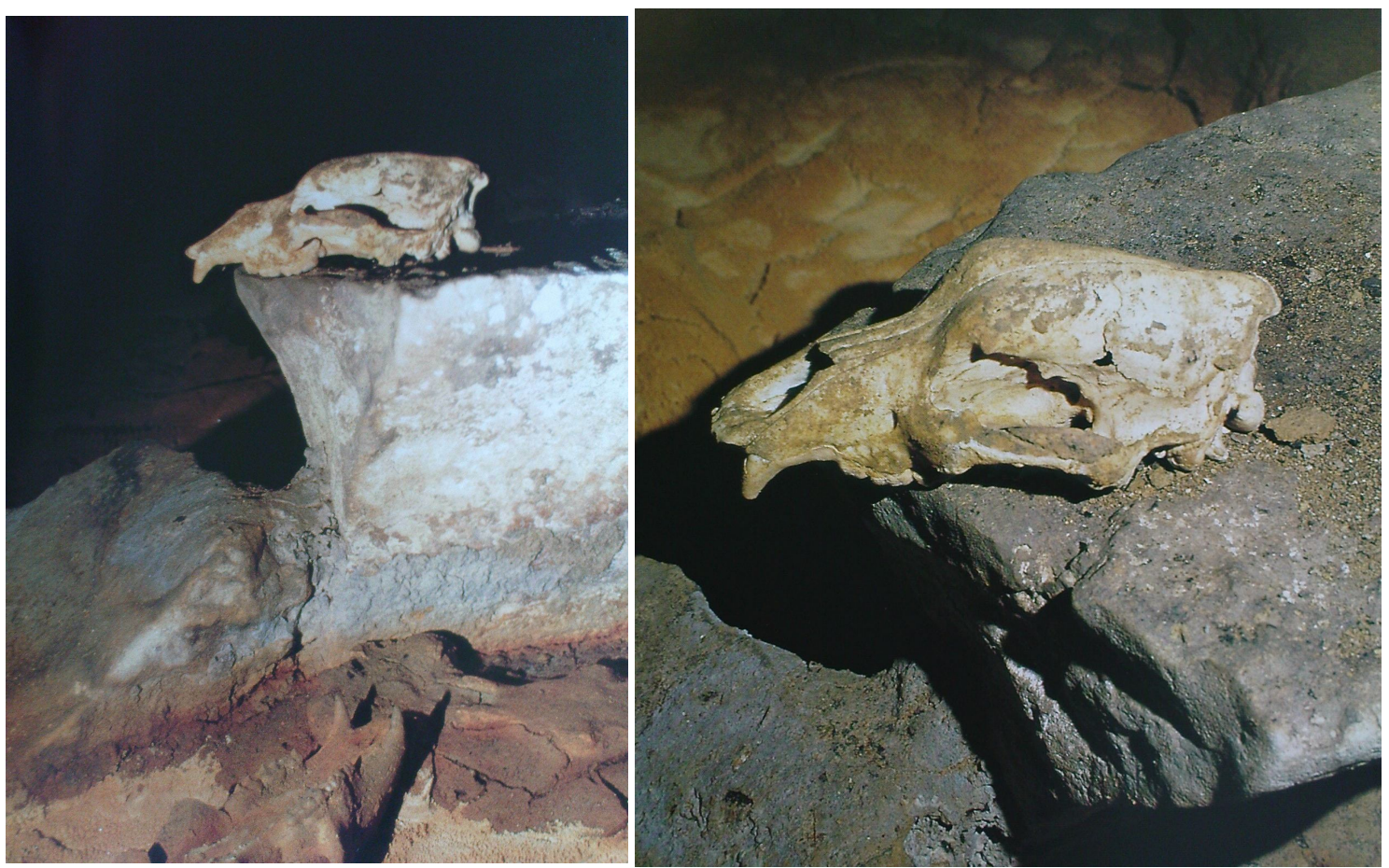

Figure 2.3 A cave bear skull placed on a fallen rock in Chauvet cave; many other bear skulls were found nearby (Clottes 2003)

In support of the theory, Clottes refers to data from the caves that indicate there were just a few artists. But whilst it is true that analysis of handprints and footprints supports this claim, the data also shows that the owners of these prints were a mix of ages, and of both sexes (White 2003; Guthrie 2005). And Guthrie highlights that this evidence also indicates that only a few people ever visited the caves - and if the caves were very rarely used, then the idea of shamans entering caves regularly with followers to conduct group rituals is just not credible (Guthrie 2005). Furthermore, Bahn, points out that although some of the art in deep caves is located in chambers capable of entertaining a crowd and thus a ritual, a lot of it was in small areas that were hard to access, suggesting that it was created for private use (Bahn 1998).

The art of modern-day hunter-gatherers such as the San and Australian Aborigines is different in both appearance and subject matter to the Paleolithic cave art of Western Europe; the contemporary drawings contain images of small animals, snakes, and many representations of complete humans, all of which are quite unlike those of the Paleolithic. This difference in both subject matter and style indicates that the artists did not have similar psychologies. The reliance on ethnographic comparisons therefore adds further doubt to the creditability of this theory. Furthermore, LewisWilliams' theory provides no insight into the motivations behind the vast majority of images that were made outside of the caves. At best, this theory might explain why a small proportion of 
Paleolithic cave paintings were made; from his investigations, Randall White believes that it may be as little as ten per cent of the total (White 2003).

\subsection{Adolescent Males}

\subsubsection{R. Dale Guthrie}

As discussed previously, cave art was often found deep in caves and this inaccessibility may indicate that it was a relatively personal inspiration rather than for the purpose of any group or ritual use. R. Dale Guthrie (The Nature of Paleolithic Art, 2005) suggests exactly this: that a high proportion of cave art was produced by adolescent boys whose minds were full of adult ideas, namely sex and hunting. Guthrie extensively analyses the available data from the Upper Paleolithic and identifies any consistencies and regularities that support this claim.

Guthrie argues that there is strong evidence to show that most cave art was not the end result or byproduct of religious experience and modern interpretations of this nature are biased. This bias began with the early experts being members of the clergy, and the caves being located in traditional Catholic countries (Guthrie 2005). This propensity is further reinforced by the modern idea that caves are mysterious and menacing places (Bahn 1998). Instead, he argues that what cave art demonstrates, is that the artists had a high degree of awareness of their surrounding environment (Guthrie 2005). Whilst some of the Paleolithic images may have been religiously motivated, he says, they were in the minority. Furthermore, the attempt to find symbolic meaning in cave art has, in his view, greatly detracted from the process of unbiased investigation and has stifled other lines of enquiry (Guthrie 2005). He believes that Paleolithic art lacks meaning and is purely representational (Guthrie 2005). And on the issue of why painting and other artwork occurred during the Paleolithic in the first place, he only says that we are unlikely to ever know (Guthrie 2005).

Much has been made of the art found in deep caves, but Guthrie believes that it is only a coincidental fact as this form of Upper Paleolithic art is the most likely to have been preserved. Cave art is just a small portion of all of the Paleolithic art that once existed (Guthrie 2005). Evidence of most artistic behaviour, such as dancing, storytelling, and even clothing, has been lost over time. Referring to what he calls 'Sieveking's Law, ${ }^{9}$ Guthrie points out that despite consistent

\footnotetext{
9 In honour of the work carried out by archaeologist Ann Sieveking
} 
assumptions to the contrary, ${ }^{10}$ the evidence tells us that caves were not often frequented and that very few people ever ventured back into the potentially dangerous deep caves. A lack of lamp soot on walls or ceilings, no well-trodden trails, and limited debris all support this claim. When comparing this data with population estimates, Guthrie believes that maybe as few as one Paleolithic person in a thousand ever entered deep caves and created art there (Guthrie 2005). Archaeological research shows that Paleolithic people used either abris or the mouths of caves for shelter, and they did not live in or regularly frequent the inside of caves (Guthrie 2005). Unfortunately, due to exposure to the elements, only a small amount of art from these locations has survived. Taphonomic processes, such as wind and rain erosion, preserve almost no art outside caves.

Guthrie is critical of the high quality 'coffee table' cave art photos that are chosen for books on the subject and says that from his own experience, they are not always that good (Guthrie 2005). He claims that many of the paintings, especially those in deep caves, are of a poor quality, often being incomplete or containing many overlapping lines, indicating that these paintings were made quickly and over a period of at most a couple of visits (Guthrie 2005). It also hints of a novice at work (Guthrie 2005). He therefore suggests that these paintings were largely the result of spontaneous acts and supports this claim by pointing out that many paintings were based around suggestive marks, indicating that specific images were not pre-planned (Guthrie 2005).

So who were the artists? Some of the best available evidence of the identity of who entered the deep caves can be gained from the handprints and footprints found there, and a 'significant segment' belonged to adolescents (Guthrie 2005). André Leroi-Gourhan had also noted that young adults made almost all the footprints he found, but explained this away as indicating that it was due to numerous initiation ceremonies (Ruspoli 1987). Producing extensive statistical analysis, Guthrie claims that the average age of the people who made the handprints in caves was between 10-16 years of age and mostly male. Furthermore, an analysis of techniques and styles indicates that only a few people at each location made cave paintings - Clottes also makes this observation, but uses it to draw a quite different conclusion (see above). Based upon his evidence that it was mostly adolescent males who entered the deep caves, Guthrie deduces that they also produced most of the paintings (Guthrie 2005).

${ }^{10}$ He specifically attacks the work of Lewis-Williams 
To further support his argument, Guthrie emphasises that the populations were much younger during the Pleistocene than today, and the majority of the population were in their twenties or younger (Guthrie 2005). But there would still have been a significant number of older people. Due to the harshness of life, children of the Upper Paleolithic would have been exposed to adulthood from a young age. They had to learn quickly and the role of play in a child's life was extremely important in promoting learning, experimentation, and training for adult life (Guthrie 2005). Artists learning their trade may well have been tempted to sneak off into the rear of a cave to try out their newly honed skills - they would also be more curious than adults who would have steered clear of the possible hidden dangers within a cave. ${ }^{11}$

Guthrie speculates that imaginative Paleolithic children spotted similarities between topographical features of the cave walls that were highlighted by the shadows of their torches and certain animals that stimulated them (Guthrie 2005). Scientific evidence also shows that younger brains have a much more active imagination than adults (Guthrie 2005). The subject-matter of the images drawn was quite selective - not those of small creatures but "parts of large animals, game birds, large fish, naked women, a scatter of ugly faces, and erections and vulvae" (Guthrie 2005, p. 135) - just the sort of images that would be on the minds of young males. Many of the large animals were drawn bleeding or wounded by spears, which might provide an indication of the hunting aspirations of the male adolescent artists. Guthrie highlights what Jean Clottes has reported: the amount of detail in some of the paintings and engravings was well beyond what was required, an indication in his opinion that there was possibly an element of gratification gained from their creation (Guthrie 2005). Adolescents may have had a similar motivation for producing their art.

\subsubsection{Analysis}

According to this theory, adolescent males, on adventurous expeditions underground, produced a large amount of cave art. From an evolutionary perspective, this explanation argues that cave art was a by-product of a young Paleolithic male's willingness to explore, combined with expectant thoughts of his forthcoming adult life.

There appear to be several proximate causes producing this type of behaviour by adolescent males: risky thrill seeking, exploring the unknown, and satiating growing sexual urges. Whilst caves are potentially dangerous places, they are of interest to the curious minds of adolescent males who are

\footnotetext{
11 There is even a cave art-related story to support this adventurous nature: Lascaux cave was discovered by young boys who went out hunting for a secret underground passage to an old manor house and stumbled upon the cave when looking for their dog that had run off. See page 188 of Ruspoli 1987
} 
willing to make adventurous forays into new and potentially exciting locations. Adults might practice a high degree of risk-avoidance, but young males are more carefree and actively seek out adrenaline-fuelled activities. An increasing sexual awareness might also mean that some of the images drawn on cave walls amount to a kind of Paleolithic playboy magazine where fantasies could be played out away from prying adult eyes.

The ultimate cause of possessing a curious nature may be evolutionary advantageous for young human males of the Paleolithic, by providing them with a better preparation for their forthcoming adult life. A willingness to explore their environment enabled them to learn about it, as well as their physical and cognitive capabilities within it, giving them an improved chance of survival when engaging in more dangerous activities such as hunting large animals. An interest in sex as a young male approaches manhood is also evolutionary beneficial because the earlier a sexually mature male is willing to mate, the greater the likelihood of getting his genes into the next generation.

But a possible problem for Guthrie's theory is access to materials. Before setting out on their adventures, the young artists would have first needed to acquire the necessary resources for creating art in the deep recesses of caves. In such a harsh environment these materials may have been hard to come by, and only accessible to adults. If this was the case, then cave art was more likely to have been a group activity due to costs. Guthrie fails to address this issue.

An additional criticism is that his book consists of many of his own sketches of cave art - and there are no original photographs. This leaves Guthrie open to the criticism that many of his interpretations of the more ambiguous drawings are biased towards his theory; the reader is not offered the opportunity to make their own interpretations of the more controversial images.

\section{ART AS AN ADAPTATION}

Producing cave art had considerable cost - from an evolutionary point of view this type of behaviour persisted over a time and needs explaining. As Bahn points out, cave decoration required strong motivation, and a large amount of effort; it needed materials for painting and illumination, and involved the potentially hazardous negotiation of cave networks (Bahn 1998). So was it an adaptation? 
Paleolithic behaviour of fitness-gaining activities such as the manipulation of fire or the manufacture and use of tools, are clearly suited to evolutionary explanation. Attempting to explain the behaviour of those who created cave art, however, seems somewhat more puzzling. Both the planning and execution involved in the production of cave art required lots of effort, so it appears unlikely to be a kind of Paleolithic doodling (Boyd 2009); it must therefore have been important and functional to those that participated in this activity.

A prerequisite for making art is the availability of time. It seems reasonable to assume that before our Paleolithic ancestors began creating elaborate and time-consuming paintings, they first had to become efficient hunter-gatherers with an amount of spare time. This assumption may not be so clear-cut however, as it has been reported that some modern-day hunter-gatherer groups continue art-making even during times of extreme hardship (Boyd 2009) - so for at least some, the desire to produce art must be strong. Furthermore, if creating art was a night activity, then the opportunity cost may have been low.

Most animals with long periods of 'downtime' - and especially apex predators such as lions choose to sleep and rest. Yet Cro-Magnons chose to do otherwise. Resting is a very good lowenergy way of using up spare time, whereas art-making usually requires a considerable amount of time and effort. The methodology of Darwinian natural selection can be applied to how an animal makes use of its spare time - not just what an animal does when it is being active: animals that hibernate and sleep do so because it in some way improves their ability to survive (Boyd 2009).

Seemingly futile activities have their benefits: for example, grooming reinforces social bonding and hierarchy, which in turn promotes cooperation, and so improves group survival. Similarly, it seems that for art to have persisted over time, it must have provided some benefit to fitness - if it didn't, then all the effort involved would be a weakness in those humans who participated in it and over evolutionary time, would not be selected for by evolutionary processes. The anatomically modern humans who used their time for more productive activities, or for simply resting, would have prevailed (Boyd 2009).

\subsection{Sexual Selection}

\subsubsection{Denis Dutton}

The most commonly used example of sexual selection is the peacock's tail: it is incredibly extravagant and appears to serve no particular function. Additionally, it reduces the possessor's 
chances of survival by highlighting its location to predators and impeding movement. It is also biologically expensive to grow. Natural selection pressures should ensure that other peacocks with less flamboyant plumage survive better than their glamorous cousins, and over time replace them within a population. But an extravagant peacock's tail demonstrates to a peahen that its owner is strong enough to survive despite all these handicaps and will therefore produce healthy offspring. Thus, those peacocks with the grandest tails have an improved chance of mating because they attract more peahens and their genes will be passed on to the next generation. The increased reproductive success of peacocks outweighs their reduced chances of survival.

The evolutionary psychologist Geoffrey Miller argues art is an adaptation that originated through a process of sexual selection. He claims that participating in artistic behaviour improves an individual's evolutionary fitness by increasing their mating success (Dunbar, Knight et al. 1999). Miller supports his argument by referring to a survey he carried out that analysed data from 16,000 diverse pieces of art. The results showed that there was a strong bias towards males during their 'mate-seeking' years (late adolescence until their thirties) (Dunbar, Knight et al. 1999).

In his 2009 book, The Art Instinct, Denis Dutton attempts to view art through an evolutionary lens and argues that it was in part driven by Darwinian sexual selection. Dutton doesn't directly address the issue of cave art, but does at times allude to it. The creation of art is instinctive he says, and he leans heavily on the work of Miller in supporting his own argument. Just as language is a human universal, he claims that art is too: all humans have an urge to express themselves artistically and it is their different cultures that affect how this artistic behaviour materialises (Dutton 2009).

Referring to the considerable research conducted into what type of landscape the people of today prefer, Dutton highlights how alike calendars from around the world are. Their pictures all have similar themes, which Dutton argues can be tied back to human evolution during the Pleistocene. The most popular landscape features are of open, grassy areas with some trees, and near water areas where our Pleistocene ancestors were most likely to find a plentiful supply of food, whilst also being able to detect and evade predators (Dutton 2009).

All humans have evolved the same core aesthetic tastes, and evolutionary psychology together with empirical evidence and observation can be used to reverse engineer the brain. These tools can be used in an attempt to discover adaptations that occurred in the Pleistocene that now produce the complex workings and preferences of our modern-day minds. Steven Pinker has argued in his book The Language Instinct that language acquisition is innate in humans, and Dutton says it is the same 
for art. The passion to produce art is instinctive; it occurs spontaneously around the world and throughout human history (Dutton 2009). The ability to communicate has obvious adaptive advantages, and Dutton argues that art has many benefits too.

Highlighting the connection between art and status and asking why we look up to great artists, Dutton argues that it is because art is linked to, and driven by, sexual selection. Creating art is a way for an artist to demonstrate their general fitness to potential mates by showing that they have adept mental and physical capabilities, as well as the spare time and access to resources. Art is a display of skill, and sexual selection helps explain the more flamboyant side of art - it's showing off to impress potential mates (Dutton 2009). Indeed, when reviewing many forms of art, male artists do appear to have the power to attract females - one only has to note the long list of the average rock musician's conquests, and Picasso was well known for his fondness for the opposite sex.

But Dutton argues that art is more than just a product of sexual selection, it's an adaptation of the mind that has evolved from the pressures brought about by several evolutionary forces. The human art instinct is not the same as our liking for sweet and fatty foods, he says; it is a set of 'subinstincts' (Dutton 2009). Factors influencing our urge to make art include responses to aesthetic preferences, an active imagination that allows for and creates a flexible behaviour to improve survival, sociality and social status, the solving of intellectual puzzles, demonstrations of skill, power, and wealth, and also mate attraction (Dutton 2009).

Determining genuine biological adaptations can be a difficult task because they may instead be either the result of a one-off genetic mutation, or a side-effect (by-product) of one or more fitnessenhancing adaptations (Dutton 2009). Behavioural adaptations - instincts - are even harder to ascertain because their function is not always as evident as their physical counterparts. The roots of artistic behaviour may be complex, says Dutton, but they still have an evolutionary history. As human artistic tendencies have persisted over time, they are not the result of a random mutation (Dutton 2009). He dismisses Stephen Jay Gould's argument that the psychological adaptations that have produced human culture (including artistic behaviour) are all by-products of one adaptation: the over-sized human brain. Spoken language is not a by-product, but a genuine adaptation of that same large brain, and Dutton is critical of Gould's failure to mention this in his work (Dutton 2009). Because art, like language, appears spontaneously across all cultures, it too must have some sort of fitness advantage, he says (Dutton 2009). It is an adaptation that is now innate (Dutton 2009). 
Instead of Gould's perceived negative methods, Dutton prefers how Steven Pinker has analysed human prehistory by examining our evolved psychology, and he believes that this is a much more constructive way of conducting research into our evolutionary past (Dutton 2009). But like Gould, Pinker also claims that the arts are an evolutionary by-product. In response, Dutton argues that it is too simplistic to neatly divide an organism's features into either adaptations or by-products. A highly evolved organism such as a human has a long and tangled evolutionary history. Over time, an initial group of core adaptations have - sometimes by combined influence - produced a series of additional adaptations or by-products. Adaptations may be further enhanced and by-products utilised, again as a result of several factors. This ongoing process produces a group of interlinked adaptations and by-products, and a complicated mix of evolutionary changes (Dutton 2009).

So a feature that makes use of an effect of a by-product should not necessarily be treated as a byproduct as well. Nature may often take advantage of an inefficient solution to a problem and put it to use for some other purpose that results in an improvement in fitness. A good example of this is feathers: originally an adaptation for heat regulation, they later became an integral part of bird flight. Dutton insists that one needs to understand the parts that make up the system as a whole; each part is interconnected (Dutton 2009). Subsequently, because many behaviours are the product of combinations of these interlinked adaptations and by-products, their motivation is not always easy to deduce. Dutton has argued that the motivation behind artistic behaviour is equally complicated.

\subsubsection{Analysis}

As mentioned in the previous chapter, possibly one of the earliest forms of 'art' were the beautiful Acheulean handaxes from up to 1.4 million years ago. Vast numbers have been found in some locations and many were unused, and it has been speculated that the motivation for producing them was to attract mates (Kohn and Mithen 1999). If this is true, then Darwinian sexual selection was at least in part responsible for some of the earliest art and lends support to Dutton's theory.

But the strongest evidence against this argument is that art is also produced by females as well as males, and by males across all age groups. This obviously contradicts the claim that the purpose of artistic behaviour was for males to attract a female during their mate-seeking years (Boyd 2009). Although the analysis by Miller does show that there is a strong bias towards the male 'mateseeking' group - but there may be other explanations for this, as I mention below. 
So can we find any link between cave art and sexual selection? Although cave art is one of the earliest forms of visual art, a possible link to it being an adaptation to gain sexual favour seems tenuous at best. Cave art was often drawn in the most inaccessible of locations, deep within caves where it's hard to reach, indicating that it was more of a personal than joint activity. This contradicts the sexual selection argument, for just who was the artist 'showing off' to? The only possible response would be to claim that the creation of such isolated art was used purely for the purpose of practice, before demonstrating the refined technique to others. If we instead focus on the parietal art occurring outside of caves, or at least in the larger and more accessible chambers of a cave, then Dutton and Miller's hypothesis may be slightly more credible, if still a little unlikely.

I would tend to agree with the thoughts of Brian Boyd, that sexual selection is more likely to have been an additional use for art, but not necessarily the initial driving force (Boyd 2009). The example of the Acheulean handaxes does, however, suggest that art and sex might have been closely linked since an early time in our evolutionary history. There are many possible explanations why more men create art than women, but rather than there being a link to sexual selection, it may be a simple case of men being more competitive and thus a reflection of their dominant position in the world (Boyd 2009).

\subsection{Patterned Play}

\subsubsection{Brian Boyd}

In his book On the Origin of Stories (2009), Brian Boyd discusses how art in general (including narrative, dance, song, and drawing) may have evolved. Whilst his particular focus is on the art of storytelling, he does on occasion specifically refer to cave art. Producing art involves cost - time, energy, and resources. This cost occurs over generational timeframes and requires explanation. Boyd argues that art derived from play and is a biological adaptation that enhances fitness by improving the bearer's chances of survival and reproduction.

Before discussing his own theory on the evolution of art, Boyd explains why he strongly disagrees with those who say that it developed out of religion. To support this claim, he points out that there are no signs of grave ritual earlier than 90,000 years ago (a clear sign of religion), yet ochre appears to have been used for body decoration for at least 120,000 years - if not twice as long ago (Boyd 2009). However, as I have previously stated, ochre had other uses too, such as its medicinal properties for treating wounds. Boyd also refers to an earlier time in human history, when the 
ancient handaxes of the Lower Paleolithic were created either too large for practical use or far too symmetrical and refined for their purpose - which he argues is a further hint of aesthetic appreciation well before any indication of religious thought. Religion needs art, he says, but art does not need religion (Boyd 2009). Additionally, he notes that some animals display forms of art such as song, but are obviously not religious - although this seems to be a very weak analogy. Art does have multiple functions including play and social coordination, and whilst Boyd admits that there was a time when one of art's functions was a strong link to religion, this has now diminished in the modern era. Art and religion do share some common ground: they are both tools for improving group co-operation and cohesion (Boyd 2009).

Whilst accepting that it's impossible to know for certain what cave art meant to those who created it, Boyd argues that the artists were well aware of what they were drawing, namely animals. The drawings required considerable effort and as Boyd says, "the wall markings were hardly the casual doodles of idle afternoons" (Boyd 2009, p. 8).

Boyd strongly believes that an evolutionary model can provide a complete explanation of the origins of art. Art has both a biological and cultural background, he says: it is universal throughout the human race and emerges very early in a human's life, but it is also strongly influenced by tradition as well (Boyd 2009). The high costs involved suggest that it must have some fitness advantage for it to have perpetuated. Although artistic behaviour suddenly comes to prominence with the 30,000-year-old cave paintings of Chauvet, Boyd argues that the propensity to produce art probably dates back hundreds of thousands of years. Indeed, he speculates that it may have all started with music (which needs no additional props, and is also created by other animals including birds and whales), followed later by dance (Boyd 2009).

Returning to the use of ochre for body decoration, Boyd believes that it seems highly likely that this was how visual art began (Boyd 2009). From an evolutionary perspective, he acknowledges that any form of body decoration - or any other visual art for that matter - that enhanced biological signals, for instance sexual cues like red lips, would probably have had some degree of sexual payoff. But Boyd argues that this is not the same as sexual selection. Instead, body decoration would have been socially motivated, and used for group identification rather than individual practice (Boyd 2009). Thus, he believes that the sexual aspect of art merely piggybacked on top of its original role. 
Boyd attacks Steven Pinker's claim that art is an evolutionary by-product of the mind rather than an adaptation. Berating Pinker's only concession that narrative may be adaptive as it is useful in examining various scenarios before acting out one of them, he asks why then is art often far more elaborate than can be explained by simple decoration designed to push a few aesthetic buttons (Boyd 2009)? There must be an explanation for all the extra effort and additional cost, he says. Furthermore, if art had no survival benefits then the amount of time and resources involved would produce a burden on any group that participated in this type of behaviour and reduce their overall fitness. Artistic groups would, over evolutionary time, be less prosperous than those that were artfree, and selection would favour those lacking an artistic disposition (Boyd 2009).

The impulse to create art is universal across all humans, claims Boyd. To support this, he highlights how young infants enjoy such things as being sung to and they are attracted to shiny and bright coloured objects. This, he says, suggests that artistic behaviour is innate rather that simply culturally driven (Boyd 2009). Furthermore, the act of making art (for example, storytelling or music-making) creates pleasure for all who participate, and Boyd believes that a key factor of this type of behaviour is the ability of humans to share attention in a group-like fashion (Boyd 2009). I discuss this human attribute in detail in the next chapter.

Along with many other animals, humans play. Play allows participants to practice in a safe environment where they can hone skills that will later enhance their chances of survival by improving reaction times and the accuracy of the task required (Boyd 2009). Human play also enables the participants to learn to anticipate what their playmates are intending to do - it facilitates the development of the capacities for empathy and mind-reading. The pleasure derived from play ensures that we engage in it, and most biologists believe that play is a selected for adaptation (Boyd 2009). Art provides humans with the opportunity to practice sounds, movements, visualisations, or representations, and in the case of story, simulate possible future scenarios (Boyd 2009). What Boyd argues, is that art is a kind of cognitive play (Boyd 2009).

Humans have a hunger for information and often share what they learn with their peers. In this search for information, humans actively seek out patterns in their environment (Boyd 2009). This cognitive feature is a crucial part of a human's superior intelligence: spotting a pattern allows prediction making, and if it can be done before a competitor then it provides a key survival advantage. Art attracts human attention by activating the pattern-hunting part of the brain. Furthermore, art is a social and individual mechanism for stimulating and encouraging the 
imagination - it promotes creativity (Boyd 2009). Art is a human adaptation that derives from play. Art is cognitive play with pattern (Boyd 2009).

Boyd points out that art requires the artist to focus their attention. This cognitive skill is essential for learning, and art nurtures this ability. Humans are also able to share their attention and as he says, "to explain art we need to attend to attention. Art dies without attention" (Boyd 2009, p. 99). Shared attention is a prerequisite for group cognitive play, and art does this by grabbing your attention. Art encourages humans to share attention in coordinated ways, which in turn improves sociality; collective ritual enhances group cohesion (Boyd 2009). This collaborative bonding greatly enhances survival activities that require group coordination.

Humans are very good at working together in groups. Cooperation is a powerful social tool because it enables many actions that a lone individual would be incapable of, for example, the hunting of large animals during the Paleolithic. Groups are also better able to collectively defend themselves. So there are obvious adaptive advantages to group cooperation and Boyd argues that participating in art-making activities promotes group bonding and social activity, which in turn will improve fitness. Some researchers even argue that group cooperation has been the main reason for the huge advances in human intelligence (Boyd 2009).

Although I discussed earlier how R. Dale Guthrie believes that the majority of Paleolithic cave art is the work of adolescent males and a by-product of their hyperactive minds, he too thinks that the origins of art derive from play. He says that play is a universally accepted human adaptation: "art making is a uniquely evolved offshoot of play" (Guthrie 2005, p. 374). According to Guthrie, play was selected for because it provided a fitness advantage, by training the young for activities such as hunting. It also improves cognitive development, agility, and coordination. And like Boyd, Guthrie argues that art evolved out of play as a method of encouraging human creativity (Guthrie 2005).

Highlighting the fact that not all animals play, Guthrie says that "playing is observed in animals whose niches require versatility" (Guthrie 2005, p. 377). It has only evolved in animals that have to be flexible and opportunistic; a good example of this are ravens, who do play, whilst many other birds with inflexible lifeways do not. Most birds have relatively fixed diets, but ravens are much more flexible and make the most of any opportunities that come their way, including scavenging and predation (Guthrie 2005). Humans evolved by continuously resolving complex problems caused by an ever-changing environment - each generation's increasing cognitive ability and flexibility was scaffolded on top of earlier intelligent behaviour, he says. The imagination is the 
biggest playground of all, and cave art, storytelling, and story-making provided humans with an outlet for the fantasising minds of early hominids that could be consciously controlled (Guthrie 2005).

\subsubsection{Analysis}

Boyd's thesis provides a nice explanation of how artistic behaviour might have evolved. It also provides strong evidence to support how it was advantageous to those who participated in it and that its benefits improved their chances of survival. If Boyd's hypothesis is correct, then art is an adaptation that developed out of play. Of course the origins of individual art forms may not be so clear-cut, and there may be a mix of adaptations and by-products at work, as well as cultural evolution. Motivations arising from sex, superstition, and play may all be contributing causes to this type of behaviour. Some of these stimuli have a fitness advantage, whilst others merely scratch psychological 'itches', or cater to our evolved 'cheesecake' taste buds.

As for cave art, Guthrie's argument lends further credence to Boyd's thesis. Children play much more than adults, and Guthrie's data that the footprints found in caves - and particularly in the deeper parts - belong predominantly to children fits nicely with Boyd's claim. Paleolithic art was almost certainly produced in large amounts outside of caves, but adventurous adolescents would have had the inclination to explore the deeper areas of caves, adults much less so. Once inside, they could create the art they had seen the adults producing in the daylight.

\section{CHAPTER SUMMARY}

Unfortunately, we are severely limited in our tools for understanding cave art and so no proposed theory will ever be definitive. One of the tools regularly used is ethnography, but the conclusions drawn from its analogies can be misleading; the risk of including unconscious bias or only extrapolating the data that fits an argument is always present. Any assumptions should take into account the extremely different living conditions during the Paleolithic, but Guthrie argues that both the cultures and the people are just too dissimilar to warrant comparison (Guthrie 2005). He further claims that the reliance on ethnography by many researchers has hindered the study of cave art. 
Whilst trying to determine the motivations of the artists is very difficult, identifying the artists is perhaps slightly easier. The best clue to the artists' identities is the many handprints on the walls and footprints on the cave floors. But only Guthrie addresses this issue in any great detail, and argues that the majority of the marks belong to adolescent males. The limited number of footprints indicates that the caves were not accessed regularly, and other evidence such as the few torch marks, also pointed out by Guthrie, supports this. So producing cave art appears to be more an individual than group exercise, and not something that was then 'paraded' to appreciating crowds.

Of the theories I have reviewed, most fail to explain all the idiosyncrasies of cave art. Guthrie, for instance, does not address the conspicuous lack of complete human representations. Throughout his book he often refers to the many etchings of human faces found on slabs in La Marche cave in France. But as I have previously mentioned, these etchings are highly controversial and their authenticity has been doubted - something he fails to mention. He does, however, point out that the faces are almost always missing apart from La Marche, but provides no further explanation (Guthrie 2005). It does, in my opinion, appear highly significant that even when representations of humans have been made - such as the Venus figurines - the faces are always left blank. But why this is the case remains a mystery.

As Randall White points out, there is so much variety in both the cave complexes and the images within them, that no single theory could explain it all (White 2003). The caves vary in both size and depth, with some being hard to access and others easily entered. As more caves are discovered, the images become ever more varied - although the overriding theme is that of large hunted animals (but not necessarily hunted at that location). Whilst this thesis is concerned with cave art, the other images created during the Paleolithic, such as the Venus figurines and the beautifully engraved spear throwers, for example, should also be noted. Cave art was part of a larger pattern of artistic behaviour that needs explaining, and in my final chapter I attempt to do this.

David Lewis-Williams and Jean Clottes avoid discussing in any detail the sexual nature of much of the art found in caves, such as the drawings of vulvas and penises. And despite the various Venus figurines that have been found, they instead concentrate on items like the 'Lion Man' statuette, which fits in with their theory. I agree with André Leroi-Gourhan's claims that the location of many of the images was significant, but not for the reasons he argues: they were often placed in a specific location not for any sort of symbolic reason, but simply because the topography of the cave wall suggested a certain animal based upon the shape or shadow that it produced. The sexual selection theory that Dutton favours doesn't provide an explanation for the paintings located in deep and hard 
to access areas where sometimes only the artist would have been able to see it. Only Boyd's theory of cognitive play seems to be able to account for most of cave art's diversity, and also why we continue to draw till this day. I also like the extremely original idea from David Lewis-Williams that accounts for how creating basic images could have started without any requirement to explain additional cognitive developments - even though I do not agree with this scenario.

So whilst many theories have been put forward in an attempt to explain why these Paleolithic people produced such wonderfully crafted representations, it may well be the case that there were several reasons, and I am personally of the opinion that a hybrid model of their art-producing behaviour is more likely to be closer to the truth of it. Of all the theories, Brian Boyd's appears the best explanation from an evolutionary standpoint. But certainly in the art of today, sex does also seem to be influential. So as Boyd suggests, the pleasures of patterned play that we experience in childhood, and the shared attention that it requires, may well have been the impetus for art, but then sexual selection could have ramped up adaptations for sociality and art (Boyd 2009). Additionally, Guthrie's theory of cave art being predominantly the work of adolescent males could be used to explain the only remaining sliver of Paleolithic painting that has survived to this day.

From the evidence so far available, it seems highly probable that cave art was but a very small sample of the visual art created during the Paleolithic, with the majority of it being created outside of the caves - R. Dale Guthrie provides a very strong argument backed up by lots of data to support this claim. It therefore seems that a large proportion of painting and engraving was done out in the open either on rocks or pieces of slate ('plaquettes'), but almost all of it has now disappeared. The discovery of thousands of discarded plaquettes in Parpalló cave in Spain gives us a small indication of what might have occurred. This considerable amount of missing data may have either undermined some of the explanatory theories of cave art - or possibly have supported them.

It also seems highly unlikely that the cave artists who produced these exquisite paintings could have attained this great skill without considerable practice. So one would have expected to find many examples of trial-and-error attempts from the periods when they honed their trade, yet most authors only remark at the amazing quality. Guthrie is a lone dissenter, and claims that many examples of cave art are of a poor quality or were rudimentary. He disparagingly refers to the multitude of 'coffee-table' books covering the subject (Guthrie 2005).

It would certainly appear very strange if there were no evidence whatsoever of an artist's learning process - the ratio of poor-to-good quality paintings should have been very high. Creating images 
out in the open would have been far easier, but unfortunately there are very few examples of this due to the exposure to the elements - nevertheless, a few examples have been found due to freak preservation conditions. Drawing out in the open would also have provided the possibility of creating an image of an animal that was within sight - a slightly easier cognitive task than drawing from memory - and a possible stage in the learning process. One explanation of this conundrum would be if the artists practiced in the open and only the most skillful were allowed into the caves. Another solution is the one supplied by Guthrie.

\section{SO WHERE TO NOW?}

The advent of this phenomenon was a truly significant advance in human cognitive development. I propose an alternative approach be taken when attempting to discover just what that important advance was. I believe that cave art offered the brain an opportunity to hone many of its developing cognitive abilities and thus had selective advantages. The creation of cave art - as well as art in general - could be performed without incurring any major risk to immediate survival (forays into the farthest-most depths excluded), whilst at the same time it improved the chances of future survival by allowing the artist to refine a variety of cognitive, social, and motor skills.

Cave art was a low risk activity that accentuated our ability to survive. Bearing this in mind, Brian Boyd's argument that art is a kind of cognitive play seems most plausible. Before attempting to evaluate my alternative approach to what cave art may be indicating, in my next chapter I first intend to analyse what cognitive skills were required for its production. 


\section{Chapter 3: The Cognitive requirements of Cave Art}

\section{INTRODUCTION}

In the previous chapter, I discussed some of the possible motivations behind why Cro-Magnons created cave art. But while we will probably never know for certain what the motivations of the artists were, we can at least be fairly sure of the cognitive competences needed for participating in this type of behaviour. So in this chapter I will investigate the abilities needed to produce the many cave paintings and carvings that have so far been found.

When evaluating these cognitive abilities, one also needs to consider whether the creation of cave art required any special cognitive skills over and above what anatomically modern humans already possessed - as shown by their utilitarian technology. Could cave art have been the result of an improvement in an existing competence, a new combination of those already present, or even the development of a new one?

\section{THE EVOLUTION OF BRAIN SIZE}

When examining the phenomenon of cave art, one also needs to consider what had been happening to Early Human brain size in the lead-up to this new type of behaviour. Based upon archaeological evidence, Steven Mithen (1996), Terrance Deacon (1997), and Roger Lewin (1998) have all highlighted the fact that over the previous 3 million years our ancestors' brain size increased threefold. There were two specific periods during which this growth occurred: firstly, there was a large increase in size from 2 to 1.5 million years ago, and then a second, relatively smaller increase occurred, from 500,000 to 200,000 years ago. Metabolically, large brains are expensive to maintain and also have birthing costs, so there must have to have been strong selective pressure for them to evolve in this way. There had to have been significant advantages associated with the possession of a bigger brain.

Various explanations have been have been put forward to explain this growth in brain size. Popular current theories are that the cognitive demands of tool making produced the first increase, whilst the 
superior social intelligence required for an increasing group size may have been the catalyst for the latter expansion (Lewin 1998). Although further discussion of these theories and others is outside of the scope of this thesis, what should be noted is that the brain size of our ancestors had reached its peak approximately 200,000 years ago, and by this time it was roughly the same cubic capacity as that of modern-day humans. The significance of this is that it would suggest that our ancestors were already cognitively advanced many years before the appearance of cave art. As previously discussed in chapter 1, Neanderthals had large brains too - slightly larger than those of CroMagnons - but did not produce any cave art. So while large brains are presumably necessary for creating paintings, they are not sufficient.

\section{THE BASIC COGNITIVE COMPETENCES}

\subsection{Attention}

Producing cave art required 'selective attention'. Animals can concentrate on matters that are important for survival - the image of a feline predator stalking its potential prey immediately springs to mind; but humans are far superior to other animals at focusing and then, more importantly, holding their attention by suppressing potential distractions. It is one thing to concentrate your attention on a life-enhancing activity, it is quite another to focus it on something somewhat less critical such as producing a piece of art. The act of creating a cave painting - which would have taken at least several hours (but often took much longer (Ruspoli 1987)) - placed a considerable demand on holding the artist's attention. The capacity to maintain attention through complex, drawn out activity without immediate reward is an important cognitive ability. It is an essential tool for learning: one needs to be able to direct one's attention towards the activity in question before one can master it.

In the previous chapter I discussed the work of Brian Boyd in his book On the Origin of Stories and how his theory addressed the issue of why humans create art. One of Boyd's most important claims is that he defines art as being 'cognitive play with pattern'. A major benefit of this type of behaviour, he says, is that it engages and rewards attention (Boyd 2009). Participating in art allows its creator to improve the cognitive skill of arbitrarily focusing their attention. 
Along with greatly enhancing the ability to learn, a second benefit of actively controlling one's attention is improved cooperation. The evolution of cooperation amongst organisms provided an important fitness advantage, and the ability to share attention improves sociality: "art offers us social benefits by encouraging us to share attention in coordinated ways that improve our attunement with one another" (Boyd 2009, p. 101). Group coordination allowed our ancestors to kill large prey, as well as undertake many other activities carried out more effectively than attempted alone. Hunting was in many cases extremely dangerous, so the ability to attend to and coordinate the actions with the rest of the group was critical for survival. Complex cooperation requires shared attention and what play (and therefore art) does is to encourage that by promoting interest in attention by producing a feeling of pleasure. Whilst it appears that most cave art was an individual practice, some paintings do seem to have been joint efforts (Clottes and Lewis-Williams 1998), and so would have required a degree of shared attention. Shared attention would have been much more important for other artistic behaviours such as dance, music, and storytelling.

\subsection{Awareness}

\subsubsection{Environmental Awareness}

Cave art clearly shows an indubitable awareness of the environment - it is one thing to make a few finger marks on a soft clay wall, it is quite another to draw an incredibly accurate, naturalistic representation of an animal. As R. Dale Guthrie says, "the evidence of Paleolithic Art...portrays people in close touch with the details of a complex earth" (Guthrie 2005, p. 10). The production of cave paintings provides a clear indication that the Paleolithic artists were both aware of their fellow animals and also that they were aware of how they could manipulate their environment to recreate images of these animals. Furthermore, the increasing environmental awareness indicated by cave art demonstrates a growing capability to decouple sensory information from where it originated - both temporally and spatially.

Other forms of art do not necessarily require the same heightened level of environmental awareness that cave art does. It could be argued that the creation of music, or the act of singing, demonstrates an appreciation of sounds and where they came from. But it could equally be claimed that the reproduction of the sounds one hears does not explicitly need an environmental awareness and may only be indicating a growing ability to copy and repeat what one has heard - without any appreciation of where that sound came from. An obvious counter to this claim would be that this also is exactly what cave art is doing: copying what one sees and then reproducing it on a cave wall. 
I would argue however that unlike reproducing sounds, there is a considerable delay and detachment between the original seeing and the subsequent drawing - and this requires a further explanation over and above the ability to simply copy something. But sound reproduction can sometimes be comparable to cave drawing, for instance if it were in the form of vocal mimicry used to lure unsuspecting prey whilst hunting - in this case the sound's production is decoupled from its stimulus; this ability also requires considerable cognitive skill.

An alternative method of artistic expression to cave art is that of storytelling and dance, but just like the production of singing and musical sounds, these acts unfortunately leave no detectable fossil trace. Our only indication of this possibility is provided by the inferences made from the observations of modern-day hunter-gatherers. It is quite probable, however, that Paleolithic storytelling and dance were used to re-enact a hunt, and if this were the case then it would provide further evidence of a growing awareness of the world.

\subsubsection{Awareness of Others}

The detailed drawings of animals in cave art clearly demonstrated great artistic skill, yet the artists rarely attempted to produce any complete or realistic drawings of their fellow man or woman, and this requires explanation. Just a few human representations have been discovered, but they contain only minor detail (Leroi-Gourhan 1982). The only drawings of an anthropological nature that CroMagnons made alluded to thoughts of sex (e.g. vulvas, erect penises, and Venus engravings) and religion/spirituality (e.g. the mystical sorcerer drawings). Depictions of either hunting scenes, or any other group activity, could be interpreted as indicating an awareness of an artist's fellow hunters or the people that were part of their community - but these too were almost completely absent. It would also seem reasonable to think that artists would have drawn close relatives, children, or even more so, their partners - people who were important to them, but they did not (Clottes 2008).

Furthermore, as previously discussed, when examining the scores of Venus figurines - the only relatively detailed examples of human representation - it seems very strange that whilst their bodies were complete (although somewhat distorted), they had no facial features whatsoever. The deliberate omission of facial features suggests that the accurate depictions of the face were either taboo or that they generated mental angst. 
The remains of Paleolithic burials appear to suggest some degree of awareness of their fellow humans - and possibly a growing awareness of life itself. But as has previously been stated in chapter 1, burying the dead may have had its practical uses (Guthrie 2005), although this does not explain the presence of grave goods at some sites. Storytelling, dance, singing, and music all have the potential to demonstrate an increasing awareness of an individual's place within the group - and these collaborative acts would also address one's social responsibilities and enhance group cohesion. Only if cave art was produced as part of a shamanic ritual would its value as a social tool come into play - but the lack of a large number of footprints around the paintings appears to rule out this possibility.

Many forms of art are joint efforts and so would need an awareness of others in their execution, but it appears that cave art was much more of an individual act. Cave art would not have required an increased awareness of fellow humans, and would not have nurtured this cognitive development. Even so, the conspicuous absence of any accurate human representations from the subject matter of cave paintings - as well as other Paleolithic art - is very puzzling.

\subsubsection{Self-Awareness}

Initially, there appears to be little or no indication from the cave paintings discovered so far that the artists were self-aware. Although drawings of a hunting scene could be interpreted as including the artist, they could also be seen as a report of a first-person observation, with the artist omitted and only what was actually observed being drawn. Unfortunately, the few known examples contain no great detail.

However, the many handprints found on the cave walls did provide the artists with a direct reference back to themselves. It will never be known what the purpose of these marks was, or what they might have meant to the artists, but as has previously been stated, they may have been a kind of Paleolithic signature (Ruspoli 1987). If this is true, then these ancient artists were telling everyone who later saw their handprints - including themselves - 'I was here!' This would be a clear indication of self-awareness. Additionally, mysterious cave drawings of the part-human, partanimal, anthropomorphs would seem to indicate a growing superstitious nature and possible thoughts of their place in the world. This is yet another demonstration of an increasing awareness of themselves as an entity. 
Additionally, by using the contours of a rock surface to produce three-dimensional effects, the artists were demonstrating intentional manipulation of conditions of awareness. This shows an awareness of awareness. So it's plausible that the artists had to be self-aware to make use of how the tricks of illumination on a rock's surface produce these effects. And if their paintings were for an audience, then they were also anticipating how others would experience that art and the world.

In summary, it appears that the Paleolithic cave artists were becoming aware of their surrounding environment, the importance of sex, and puzzling over their origins. But the subject matter of the many paintings would appear to highlight that they might not have been fully appreciative of their fellow humans, beyond that of their sexual capabilities. The identity of the artist is a key factor here: adolescents might not have had the same appreciation of others as those of a hunting age, whilst the paintings of a shamanic artist would have had yet a different focus. The manipulation of cave wall topography and lighting, however, suggests that they did possess awareness of awareness - and possibly how others perceived the world. Finally, the creation of beautiful cave paintings was a highly skilled activity carried out in a relatively inhospitable environment; it showed at the very least a good sense of bodily awareness.

\subsection{Memory}

Cave art required not only the ability to retain the memory of the images of the animals to be drawn (as representations), but then to apparently recall those representations at will. Ruspoli reports that, “no 'sketchbooks' (for example, small slabs or rock) have been discovered; the artists worked directly on the walls without reference to preparatory models" (Ruspoli 1987, p. 171). What has been noticed, however, is that preliminary sketches using charcoal, sometimes appear to have been made before the paint was applied during the completion of the painting.

Just how much control the artists actually had over their access to these internal representations is still open to question, and there are a couple of ways in which these images could have been brought to the forefront of their minds without the need for voluntary recall. A first possibility, as previously mentioned, is that the 'cognitive trigger' for the memory recall was supplied by their dim lighting apparatus shining on the numerous cracks, holes, and protrusions of the cave walls. Their torches lit the walls' contours and produced flickering shadows whose shapes suggested the outlines of the large animals that appear to be so important to their way of life; they effectively 'saw' the animals in amongst those shadows. It's possible that later on, as artists gradually honed their artistic skills and learnt to control of their recall of mental representations, they were 
eventually able to paint images directly from their memory onto relatively featureless walls rather than first requiring a mental 'prompt'. A second possibility relates to the shamanic claims made by David Lewis-Williams: drawings were the direct result of hallucinations produced by induced trance, whereby the internal representations in memory 'came alive' and this it what they then drew - there was no voluntary control.

Of these two possibilities, the latter theory alone does not fully explain the large number of paintings that made use of the cave wall contours, however, if we consider that it is possible that the protrusions provided an input into the hallucinations then this explanation becomes more plausible. In either case however, a cave wall's unique features appear to have played a significant role in the recall of the artist's internal representations. Furthermore, it may have been the case that Paleolithic artists first produced cave paintings from involuntary visions of animals, but then gradually gained the cognitive ability to control them.

The production of cave art also required that the artist was able to remember the various techniques and stages involved. It would have been a long and drawn out procedure involving paint preparation and application, as well as remembering the method for reproducing a mental image on a cave wall. But early humans of the Paleolithic had already demonstrated their ability to perform many such complex tasks, including creating a beautiful handaxe out of a piece of unformed stone. The retention and later reproduction of a detailed mental image appears to be a new innovation.

An additional type of memory required for cave painting, and which is often overlooked, is working memory. This cognitive competence is also linked to the previously discussed ability of holding one's attention whilst suppressing potential distractions. The control of working memory allowed a Paleolithic artist to focus on complex procedures that produced no immediate reward - this was also a requirement for creating complex, multi-stage artefacts including tools. ${ }^{12}$

\subsection{Symbolism}

To create cave art, the artists needed to possess a form of symbolic thinking (Deacon 1997); it involved representing images internally, and then retaining their meaning. Terrance Deacon believes that the brain of our ancestors dramatically increased in size over the past 3 million years due to their growing use of symbols - and the selective pressure for larger brains capable of

\footnotetext{
12 For further reading, see Current Anthropology (51), Supplement 1, June 2010, 'Working Memory: Beyond Language and Symbolism'.
} 
symbolic language was ramped up by the 'Baldwin effect' ${ }^{13}$ (Deacon 1997). But there is an important distinction to make between the symbolic processing required for language and what was required to produce cave art: the artists' drawings of large animals were depictive symbols, rather than the fully arbitrary symbols of language. The use of language (at least in the form of gesture) is believed to have originated long before cave art, and I discuss the subject in more detail in a later section.

One way that the early Paleolithic brain was slowly developing a capability for mental representation was through participation in activities such as song, dance, narrative, the creation of simple artefacts such as scratched bone, or the use of ochre. But Steven Mithen warns that one has to be cautious of any claims made concerning the Lower and Middle Paleolithic, due to the lack of evidence (Dunbar, Knight et al. 1999). Only the artefacts have left any trace, and those that have been found from this period are extremely ambiguous in their interpretation. He therefore believes that these Early Paleolithic artefacts, as well as activities like body painting, should be treated as merely evidence of 'protosymbolic behaviour'.

Pointing out that true symbolism is hard to define, Mithen argues that it should at least have "a degree of displacement between the signifier and the signified in terms of space and/or time" (Dunbar, Knight et al. 1999, p. 154). This is the reason, he says, why facial expressions such as a smile are not counted as being symbolic. Thus, body painting should not be classed as symbolic behaviour if it was simply drawing attention to, or exaggerating, certain body parts; it may just be the result of associative learning. So, if the ancient ochre that has been found was used for bodypainting like this, then it does not appear to satisfy Mithen's criterion, but if that same ochre was used for creating the images of cave paintings then it would be linked to symbolic thought. Separately, as I have previously mentioned, an incised piece of 75,000-year-old ochre discovered in Southern Africa in 2000 is now regarded as the oldest example of art and was therefore possibly produced by symbolic behaviour. But if the incised parallel lines were merely decorative - rather than depictive - and so did not represent anything, then they cannot be deemed as truly symbolic.

An even earlier suggestion of an emerging propensity for symbolic thought can be interpreted by the shape and size of some of the Paleolithic handaxes that have been found: as previously

\footnotetext{
13 The Baldwin Effect is a theory which proposes that those individuals of a population with a better ability to learn a new type of beneficial behaviour, will be better favoured by natural selection processes than those who either cannot learn the new skill at all, or at least not quickly enough for them to reap its selective advantages. Additionally, those individuals who are physically better able to utilise the new behaviour will gain the most from it, and so any future generations with similar physical traits will be selected for over those without them. (Dennett 1995)
} 
mentioned, they were either unnecessarily symmetrical or too large to be of practical use (Boyd 2009). One conclusion that could be drawn from these artefacts is that their creators were beginning to gain an appreciation of aesthetics and possibly how the concept of beauty could be represented. (As I will discuss later on in this chapter, symbolic thought could have even possibly started with advanced animal tracking techniques, whereby an animal's tracks gradually became a symbolic representation of that animal in the brain).

What can be said for certain is that cave art clearly demonstrated an ability to reproduce decoupled representations, whereby the creation of an image was completely detached from the location of what it represents was observed. And the capability of retaining decoupled representations is a prerequisite for the creation of cave art. So although cave art was not the driving force behind symbolic thinking, it did make use of the symbolic capabilities that the brain had developed. Only if the strange symbols found on some cave walls, such as those at Cueva de la Pileta in Spain, carried some sort of meaning to the artists would the abstract part of symbolic thinking come into play but we will never know why they drew them.

\subsection{Initial Conclusion}

The emergence of cave art clearly indicated heightened levels of certain cognitive abilities. Due to the many holes in the fossil record however, cave art may not have necessarily resulted from any particular cognitive threshold being reached, but it at least puts down a marker of how advanced the brains and behaviour of these Paleolithic people had become. Other artistic behaviours such as dance, song, and music may also have further demonstrated an increasingly complex brain, although these manifestations will never be detected. We must therefore make use of all other indicators from the Paleolithic that are available to us to piece together how developed their brains were.

Having surveyed some of the basic cognitive skills of the anatomically modern humans, one question does nevertheless arise: was there any additional cognitive skill needed for the production of cave art? In the next section I will examine what complex cognitive capabilities result from the individual competences that I have just reviewed, and how they were used in the creation of cave paintings. 


\section{MORE COMPLEX COGNITIVE COMPETENCES}

\subsection{The Evolution of Imagination}

Cave art appears to be one of the earliest manifestations of our ancestors' imagination - the application of a creative mind - but the archaeological record only provides us with a limited window into the past behaviour of the Early Human. Unfortunately, evidence of elaborate dances recreating a hunting scene, or of colourful storytelling will never be found. But possessing an imagination is what Daniel Dennett claims separates humans from other primates such as chimps (Dennett 1996) - we use it to solve the abstract problems that other species are incapable of doing.

The phrase 'creative intelligence' is a difficult term to define. Many animals can be classed as intelligent, and depending upon the criteria, some could even be said to possess a degree of creative intelligence. One might consider the ability to creatively solve complex problems as a guide, and in this case it is not just primates, but also corvids that have been shown to be incredibly adept at this task whilst making use of improvised tools. ${ }^{14}$ I believe that creative intelligence requires several key cognitive abilities, including the initial recognition and analysis of a problem, the mental simulation of imagined solutions (rather than by trial-and-error), the possible use of improvisation, and planning. Cave art clearly required planning, and recalling animals previously experienced needed an imagination.

Steven Mithen's theory of how a brain becomes capable of creative intelligence is what he calls 'cognitive fluidity' (Mithen 1996) - an idea that I have previously mentioned and find persuasive. Cognitive fluidity is the ability of a brain to exchange knowledge between its specialised domains rather than them being isolated from each other. The possession of specialised domains may demonstrate intelligence and skill in particular areas of behaviour, but cognitive fluidity facilitates a more flexible - and creative - behaviour. The depiction of non-actual, fictional beings, such as human-animal hybrids for example, demonstrates the cave artists' ability to combine thoughts on different subjects (as a result of cognitive fluidity).

Mithen argues that the possession of creative intelligence, rather than the use of material culture, was the crucial factor in our ancestors' survival and prosperity - whether or not it was dependent

\footnotetext{
14 For more on this, see Emery, Nathan J. and Nicola S. Clayton (2004). "The Mentality of Crows: Convergent Evolution of Intelligence in Corvids and Apes." Science 306(5703): 1903-1907.
} 
upon language (Mithen 1998). Creativity allows the recognition and solution of problems, but the subject of human creativity has been mostly overlooked in the plethora of writings about the evolution of language, he says. (In chapter 4 I will address the issue of how much of an effect the creation and subsequent use of material culture had upon our ancestors' increasing intelligence).

A combination of the earlier mentioned cognitive skills and in particular the ability to control memory access, as well as to hold symbolic thoughts, are prerequisites to the development of a creative mind. The capability of holding internal representations and then manipulating them would also be required. An adept memory that would allow such things as the pre-planning of something non-present such as a hunt would be another. At the very least, cave art seems to show the capacity to combine and manipulate memories in the mind, and use a new representation to control action assuming that cave artists decided what to paint before executing their painting.

Other Paleolithic art also clearly demonstrates the presence of a creative mind: the carvings of animals, or the Venus figurines, are examples of representations in the brain becoming physical representations, and before they were created, the artist had to first imagine its shape in the block of stone or bone being carved. Storytelling is another cognitive competence that demonstrated the presence of an imagination: it required the ability to represent not just the memories of an actual past, but also to create a 'memory' of a possible future. Similarly, cave art required some sort of forward-planning mechanism when preparing and gathering the equipment needed to produce the images.

Another - and much earlier - skill was tracking. The tracking of an animal might have been the first case of our ancestors using an imagination (Shaw-Williams forthcoming). With the use of projectiles such as spears to hunt large animals, it would have been rare to kill them where they were first attacked - they would instead only be wounded (possibly mortally) and capable of escaping. A large, wounded animal was extremely dangerous and so any tracking would have been carried out at a safe distance. According to R. Dale Guthrie, many modern-day hunters report that they imagine the individual characteristics of an animal they are tracking based upon its footprints (Guthrie 2005). Something so critical to survival as tracking down an injured prey would have certainly rewarded those who were more adept at this skill. Selective pressures for improved tracking capabilities would have been strong. Furthermore, unlike other predators, humans do not possess the same finely tuned senses such as smell and sight, so any advantageous cognitive abilities would have been a huge bonus. For this reason, the later domestication of the wolf dramatically improved tracking abilities and the subsequent success of any given hunt. 
Just like the hunters, large animals were also on the minds of the cave artists. Cave art required an imaginative mind, and based upon the number of paintings that utilised the topography of the cave walls, the artists must often have imagined the animals they painted after seeing the shadows produced by the dim lighting they used. Art in general promotes creativity, and cave art would have been one of the activities that both stimulated and nurtured the imagination of anatomically modern humans.

\subsection{The Connection to Language}

As previously mentioned, symbolic thought was a huge cognitive advance: the ability to internally represent something, along with its associated meaning, would have greatly increased the capabilities of the human brain. Both language and cave art required symbolic thought processes. Cave art contains many images of large animals, and the coordination required for hunting them would have needed an effective method of communication due it being an extremely dangerous and complex task. The pursuit of these large beasts would have been much more successful once their hunters acquired some form of language; hunting may also have produced selective pressure for its further development.

Although there has been a great deal of debate concerning exactly when language evolved, evidence indicates that its use had long preceded cave art. Much has been written recently in support of the claim that the use of language has a long history. Terrence Deacon (1997) has argued that symbolic language - or at least symbolic thought - has been slowly evolving perhaps as far back as 2.5 million years ago when our ancestors began using stone tools. But this speculation has only limited evidence due to the dates involved and so would be extremely hard to prove. One piece of evidence in support of this date has resulted from the analysis of a 2-million-year-old Homo habilis skull, which seems to show the presence of the Broca's area ${ }^{15}$ of the brain (Mithen 1996). And after surveying a large amount of the literature on the subject and its supporting data, Sally McBrearty and Alison S. Brooks (2000) argue that there is good reason to believe that language has a deep history and that some form of it originated early in human evolution.

Steven Mithen (1996) discusses the earlier mentioned brain enlargement from 500-200,000 years ago and believes that it was linked to an improvement in language complexity. Whilst Merlin

15 An outer region of the brain strongly associated with speech. Indentations on the inside of a skull can indicate its earlier presence. 
Donald (1991) has analysed the human fossil record and claims that the physical apparatus (such as the vocal tract and larynx) needed for complex speech, was in place approximately 300-200,000 years ago. Additional evidence comes from the claim that language has a genetic base. There has been a great deal of investigation into the FOXP2 gene, which is thought to be linked to language ability, and evolved approximately 170,000 years ago (Mithen 2005).

But not everyone believes language origins are so ancient. Roger Lewin refers to the work of Iain Davidson and William Noble and their claim that spoken language is both a recent evolutionary development and that it is "closely tied to the cognitive processes of the development of imagery and art" (Lewin 1998, p. 463). But the claims by Davidson and Noble are somewhat contentious, and Deacon regards them as a product of "overzealous extrapolation from human artefacts to human minds" (Deacon 1997, p. 370).

So although cave art does not appear to have preceded language, it might have been a by-product of its cognitive core: language requires symbolic thought, and once this cognitive competence was established, the creation of non-present representations on cave walls would have been made possible. One plausible scenario is that representational art (including cave painting) and language have a common source in gesture and trackway reading (Kim Sterelny, personal communication). These two early skills required an ability to interpret and then mentally represent abstract information. In the case of language evolution, this may have gradually become more abstract and less iconic, developing into arbitrary, word-like symbols, and finally language. Whereas the path to cave art might have been via an elaboration into depictive representations that over time became detached from their stimulus, eventually allowing the possibility of fictional depictions such as anthropomorphs.

In summary, from the evidence available it seems probable that language in something like its modern form has been around for perhaps 200,000 years - well before the cave paintings of Western Europe 40,000 years ago. But cave art and language may have originated from the same cognitive capabilities. Furthermore, language facilitated the spreading of ideas to all those within earshot, but cave paintings of the Upper Paleolithic were far more enduring and could encompass a much larger audience.

\subsection{Theory of Mind}

Another higher-level cognitive ability, which may have been linked to cave art, is theory of mind. This ability is a kind of mind reading, which allows humans to predict and rationalise the behaviour 
of others by being able to accurately attribute the mental states held by them. It can also be used for analysing an individual's own thoughts and behaviour. Early humans lived in small groups but during the Paleolithic they gradually increased in size (Mithen 1996). The smaller groups would have been made up of family and close relatives who knew and trusted each other, but as numbers grew it would have been crucial to identify and remember the behavioural traits of the other members of the group. The need to interact with this greater number of cohabitants generated selective pressure for possessing the social skills that would have been essential for maintaining a group's harmony and also ensuring its survival (Mithen 1998). It is difficult to ascertain an exact date when humans gained a theory of mind capacity, but Steven Mithen argues that based upon research that observed Machiavellian behaviour in chimpanzees, they must possess some degree of a theory of mind to be able to do this - so it can therefore be extrapolated that early humans must also have had this branch of social intelligence (Mithen 1996).

Theory of mind is comprised of several of the individual cognitive skills that were mentioned earlier. Awareness: an individual needs to be aware of the other members of his or her group, as well as their own place within that group; crucially, theory of mind demonstrates an awareness of awareness. Memory: an individual needs to remember what he/she has observed and learnt about the dispositions of the other individuals they regularly encountered. Creativity/imagination: an individual needs to be able to predict the behaviour of fellow members of the group and adjust his or her behaviour in a way that gets not only the best out of the group, but even more importantly, the best result for the individual. And to make these predictions of future behaviour, the individual first needs to be good at recognising patterns - and as Brian Boyd points out, art promotes interest in patterns.

Social intelligence was an important addition to an Early Human's cognitive skills, but did an evolving theory of mind play any part in the ability to produce cave art? According to Steven Mithen it did, because possessing a theory of mind - which involves making predictions - is one of the main cognitive talents that facilitates creative thinking (Mithen 1998), a key cognitive skill for the creation of art including cave paintings. Mithen supports this claim by referring to Simon Baron-Cohen's work on autism and the cognitive and behavioural effects that this brain disorder has on those who suffer from it. Two cognitive skills in particular are lacking in the sufferers of autism: they exhibit little imagination, and they demonstrate a lack of theory of mind - both skills strongly linked with the ability of thinking creatively. Furthermore, autistics are unable to represent unreal things (Mithen 1998). In particular, Mithen focuses on the half-human, half-animal, anthropomorphic paintings from the Upper Paleolithic and draws a comparison with autistics, 
arguing that these drawings would have been impossible to create without some theory of mind capability.

However, Nicholas Humphrey disagrees with Mithen's claims. He instead argues that the possession of a theory of mind was not a prerequisite for the creation of cave art (Humphrey 1998). To support his argument, he describes the case of an autistic child named 'Nadia'. Nadia had no theory of mind, severely limited language skills, and she lacked certain mental concepts. She did, however, have a photographic memory. Amazingly, despite her cognitive impairments, she could draw, and in a style that was strikingly similar to that of the way cave art was drawn, he claims. By the age of only three she was drawing from memory and by five and a half years old she was creating complex drawings such as a person riding a horse. By comparison, healthy, similar-aged children are not anything like as technically skillful (as the figure below illustrates). Interestingly, as Nadia grew up and her ability to speak improved, her artistry waned (Humphrey 1998) - possibly suggesting that her greater reasoning ability came at the cost of her memory skills.
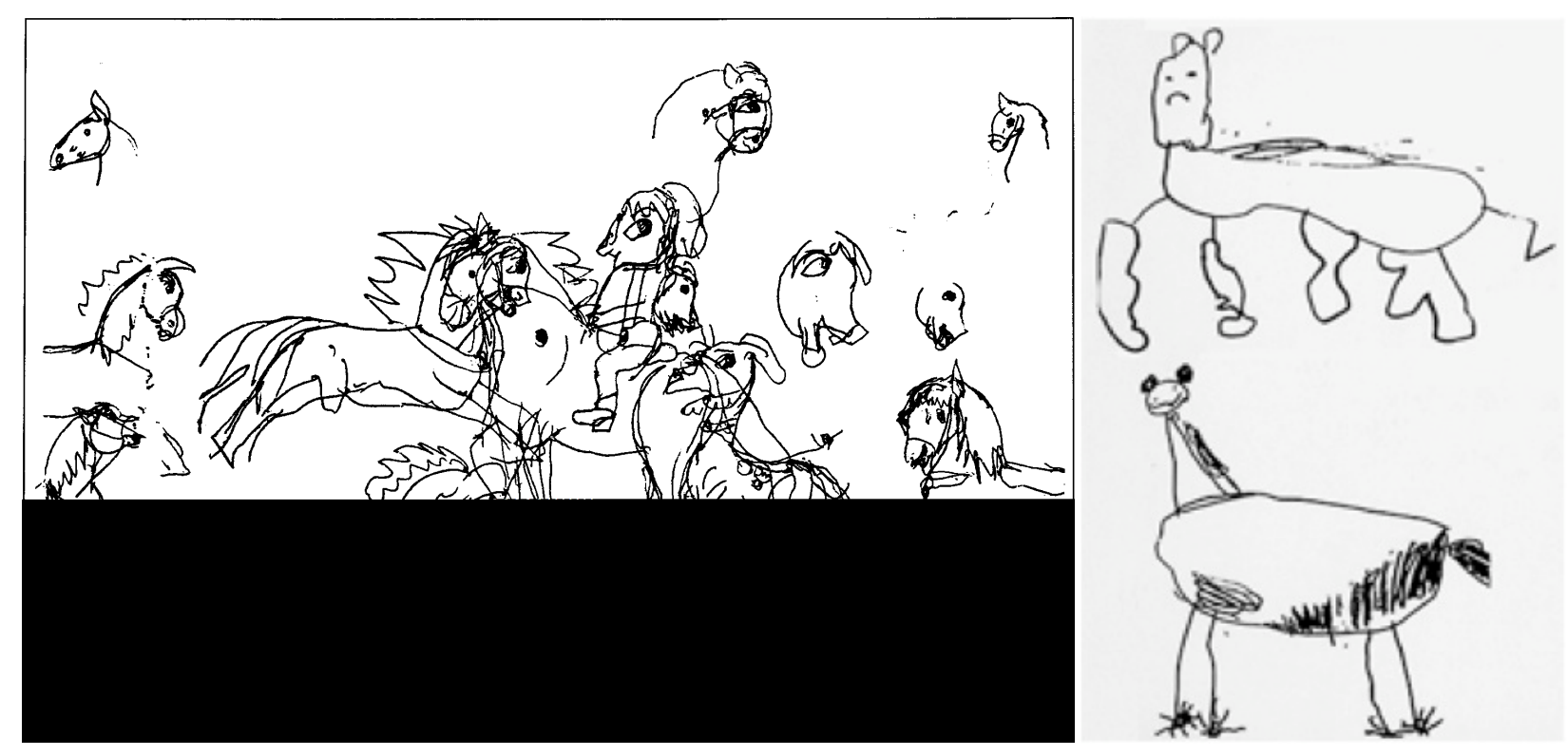

Figure 3.1 (above, left) Horses drawn by Nadia, at 3 years 5 months (Humphrey 1998)

Figure 3.2 (above, right) Horses drawn by normal 4-year-olds (Fox 2002)

Whilst an interesting argument, I believe that Mithen and Humphrey are not entirely addressing the same scenario: Mithen is arguing that a theory of mind is required to imagine a fantasy-based anthropomorph, whereas Humphrey is arguing that art can be produced by people who lack a theory of mind. They are arguing at crossed purposes and both may well be correct. In his paper Humphrey does provide two examples of composite drawings by Nadia, but it is my view that this is a very 
generous interpretation. One of them (below left) is more likely to be a drawing comprised of several sketches superimposed on top of each other, whilst the other one (below right) might just be the product of a confused mind.

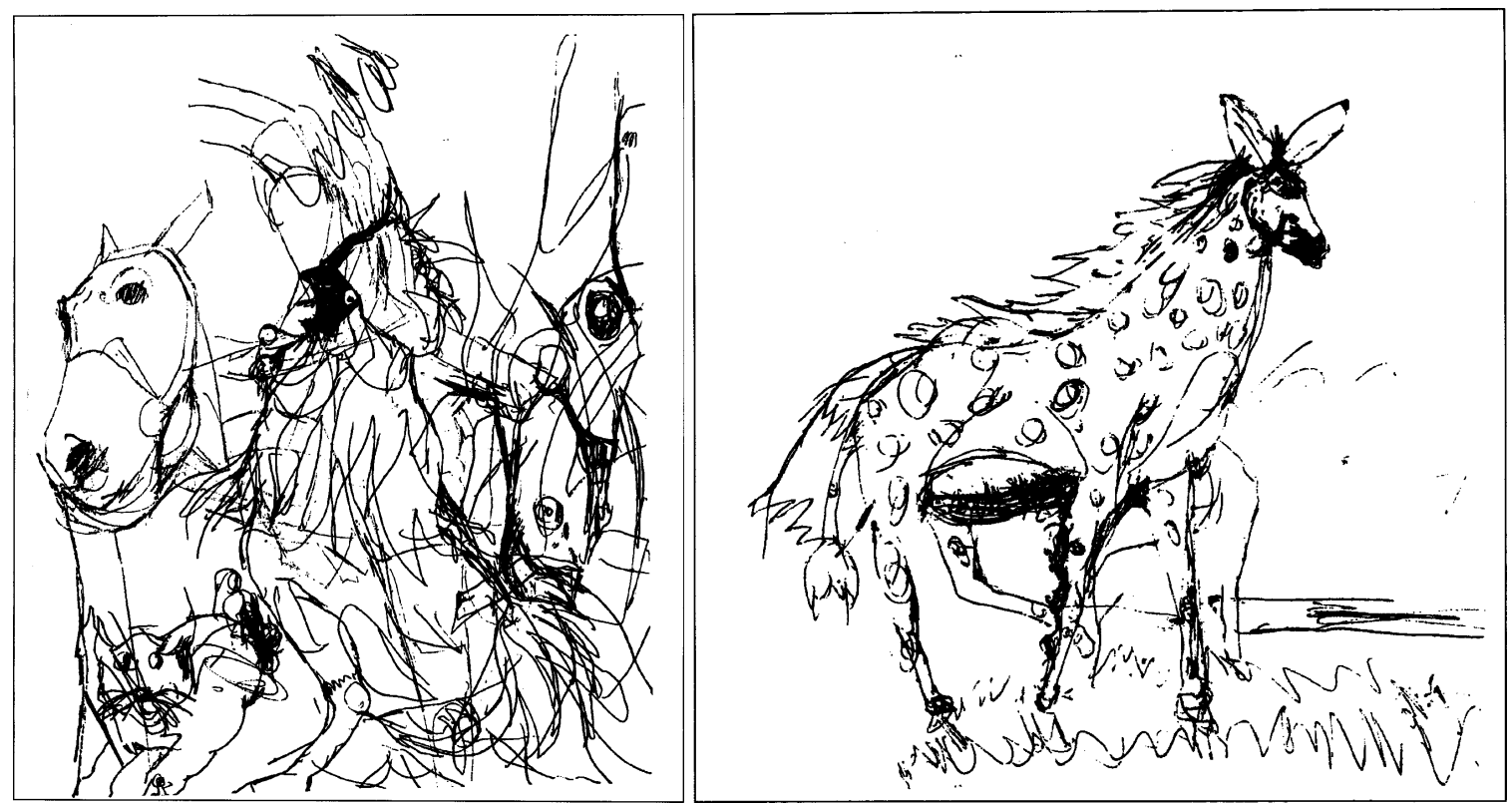

Figure 3.3 (above, left) Superimposed animals (a cock, a cat, and two horses) by Nadia, at 6 years 3 months (Humphrey 1998)

Figure 3.4 (above, right) Composite animal, part giraffe, part donkey, by Nadia at approximately 6 years (Humphrey 1998)

Gregory Currie and Ian Ravenscroft also discuss the case of Nadia in their book Recreative Minds (2002), and they too have speculated about what role the development of a theory of mind played in the creation of cave art. They disagree with Simon Baron-Cohen's interpretation of the Paleolithic paintings of anthropomorphs as indicating a full theory of mind capability, and instead argue that this feat would only have required an imagination capable of thinking of a hybrid creature (Currie and Ravenscroft 2002). And maybe the requirement of an imagination wasn't needed at all if, as the authors suggest, the hybrids were only representing real Paleolithic shamans wearing animal headdresses (which I believe to be a very plausible idea). Either way, Currie and Ravenscroft do not believe that the Paleolithic drawings of anthropomorphs clearly demonstrate that the artists possessed a theory of mind.

Without knowing the true intentions of the Paleolithic artists we cannot be sure about what the drawings meant to them. The anthropomorphs may just have been an unintentional mix of two drawings, or even if they were drawn intentionally, they may still have been accurate representations of real shamans. Similarly, the case of Nadia is open to interpretation, but it does show at a minimum that a theory of mind is not a requirement for the production of art. From the 
evidence - such as the Machiavellian chimps - it appears that our Paleolithic ancestors had long possessed a theory of mind capacity, but it is not necessarily tied to the creation of cave art.

\section{CHAPTER SUMMARY}

In this chapter I have attempted to identify the main cognitive skills required to create cave art. Initially, I wondered whether the advent of such a unique and complex behaviour was in any way linked to a change in brain size. So I investigated human brain evolution, but discovered that its size had been stable for at least 160,000 years before the Paleolithic artists began painting on the cave walls of Western Europe. Therefore cave art does not seem to have been the immediate product of growth spurt in the brain. And even the oldest known art, from Blombos cave in South Africa, was still produced some 125,000 years after the human brain had reached its current volume. So an increase in brain size does not appear to have led directly to the creation of visual art.

I next surveyed some of the basic mental capacities that would have been necessary to produce these incredibly intricate paintings. Attention, awareness, memory, and symbolic thinking would all have played their part in enabling Cro-Magnons to create cave paintings. Although none of these cognitive abilities were new, cave art would have helped to nurture them whilst also improving an individual's control over them.

I then reviewed some more complex and higher-level cognitive skills that are produced by those basic mental competences and attempted to ascertain how they might be connected with the production of cave art. Possessing an active imagination would have helped the artists to 'see' the animals they painted amongst the flickering shadows produced by their torches lighting the cave walls. Linguistic abilities almost certainly predated Upper Paleolithic cave painting by several hundred thousand years, but the symbolic thought processes involved would have enabled artists to manipulate mental representations of animals that could later be reproduced on cave walls. A theory of mind capacity may have played a part in the creation of cave art, especially the paintings of anthropomorphs. But analysis of the artistic abilities of an autistic girl named Nadia, who lacked a theory of mind, has proven to be inconclusive.

Analysis of the cognitive skills involved appears to show that no new abilities were required for producing cave paintings. Although producing cave art may not have directly improved the fitness 
of those who participated in this type of behaviour, improvements in associated cognitive skills would have proven beneficial. Cave art would have stimulated many different brain functions in a relatively safe environment; it would also have refined an individual's motor skills, including handeye coordination. Activities as diverse as the learning of hunting techniques to social interaction would have benefitted from involving oneself in cave painting.

\section{A DIFFERENT SCENARIO}

Archaeological evidence suggests that the brain of Homo sapiens had been relatively unchanged in size for approximately 160,000 years before cave art appeared in Western Europe, so rather than this event being a dramatic behavioural change, it was much more likely just one step in a gradual process of cognitive development. Nevertheless, the rate of increase in creativity and intelligence was beginning to accelerate. Whilst it is possible that the brain was reconfiguring its internal structure to generate this increasingly rapid change in behaviour, maybe this transformation was facilitated by external developments.

In the previous chapter I reviewed arguments claiming that cave art was either the result of a byproduct of evolution, or that it was an adaptation. But in the next chapter I intend to take a completely different approach and will instead evaluate whether or not it was the start of a major change in our ancestors' cognitive behaviour. Is it possible that the production of cave art signalled a 'ratcheting up' of modern human intelligence, enabling subsequent higher and more powerful levels of thought?

Rather than investigating how or why cave art occurred, maybe the question should be asked: is there any other cognitive change that the advent of cave art could be indicating? Something over and above the behaviour of anatomically modern humans at that point time. If some other behavioural change could be identified, then this might signify a sudden jump to a new level of cognitive ability. Andy Clark has hinted at a possible answer in his book Natural Born Cyborgs (2003). In a review of the book in the journal Metascience, Steven Mithen has argued that cave art and carvings may be one of the earliest examples of "freezing a thought" (Mithen 2004, p. 166). Anatomically modern humans were beginning to engineer and alter their environment, and cave art was a way of putting information out into the world rather than retaining it internally. We take this idea up in the next chapter. 


\section{Chapter 4: Cave Art: Freezing a Thought?}

\section{INTRODUCTION}

Having previously discussed (in chapter 2) the evolutionary arguments surrounding the 'adaptation versus by-product' debate on the origins of cave art, it is now time to look at an alternative possibility: that arguably cave art was the beginning - or at least an early stage - of a new type of cognitive processing. In this chapter I will explore the idea that cave art enabled the extension of human cognitive abilities out of the skull and into the world. I will examine whether cave art could have been an external breakthrough that allowed our ancestors to manipulate their environment in ways that either enhanced or complemented their inner cognitive capabilities. If this was the case, then there may indeed have been some additional cognitive attribute indicated by Paleolithic cave art that is over and above all those that were reviewed in the previous chapter.

The environment is informationally rich, and any animal that is better at evaluating that information has a distinct adaptive advantage over others that share that same environment. The ability to recognise patterns of information within the environment enables an improved negotiation of it, as well as the possibility of predicting the actions of other agents. I have previously referred to the issue of patterns when discussing the work of Brian Boyd, and how he believes that artistic behaviour is a form of cognitive play with pattern. Humans are very good at detecting patterns of information, and this may well be one of our main cognitive strengths. ${ }^{16}$ But humans not only actively seek out information, they also inadvertently or deliberately leave it out in the world as a result of their actions - and that is what I will be discussing in this chapter. Cave art may have been one of a number of methods and technologies for placing information out into the environment or a spinoff of those methods. Putting some of the brain's information into the environment would have helped to reduce the increasing cognitive load that the Cro-Magnons of Western Europe were confronting.

\footnotetext{
16 For further reading, see Daniel Dennett's book Brainchildren (1998), in which he discusses the role of patterns and the part they play in human thought.
} 


\section{PUTTING INFORMATION OUT INTO THE WORLD}

\subsection{An increasingly complex way of life}

As humans evolved during the Paleolithic, their behaviour became ever more sophisticated. Existing technologies continued to be improved upon and refined, and new ones were invented. Increasingly elaborate tool manufacture and innovations in hunting techniques would have been just some of the many valuable skills needed to improve the chances of survival. Artistic behaviour flourished. Paleolithic lifeways became more socially orientated and cultural information accrued. As human behaviours increased in their complexity, they would have produced additional demands upon cognitive abilities.

The burgeoning amount of information that had to be retained would have placed greater demands on human brains. Each subsequent generation would have been presented with an increasing difficulty of at first learning these new behaviours, and then having to remember all that they had learnt. In Western Europe however, the colder climate, ecology, and topography would have introduced additional demands on the resourcefulness of the inhabitants (D'Errico 2003). This was true for both the Neanderthals already living there and also the anatomically modern humans who arrived there approximately 40,000 years ago.

The amount of information and skill sets required for living in the harsh conditions of glacial Europe would have been significantly greater for the Cro-Magnons than the anatomically modern humans who remained in Africa. Whilst McBrearty \& Brooks (2000) have argued that anatomically modern humans in Africa were capable of cognitively sophisticated behaviour, the climatic pressures in Western Europe produced a need for a still more complex behaviour due to an increased requirement for material culture such as warm clothing and effective shelter, and seasonal variation in resources (Henshilwood and Marean 2003). The more sophisticated behaviour, combined with greater demands on knowledge accrual and the learning of new skills, would have produced an ever-increasing cognitive load; there may have been selective pressure for cognitive developments that alleviated it. Making use of the environment to supplement cognitive load would have proved highly advantageous - and cave art may be an indication of this. Additionally, it has also been speculated that the collision with Neanderthals and their culture, as well as the new challenges of living in Western Europe, were the catalysts for the sudden increase in technological and cultural complexity in Cro-Magnon behaviour (D'Errico 2003; Stiner and Kuhn 2006). 


\subsection{Complex information, and reverse engineering it}

Some Paleolithic artefacts (e.g. a spear, or a fish-trap) would have acted as templates, easing the information load, but others did not reveal their method of manufacture quite so easily. Manufacturing skills, as well as performance arts like storytelling or dance, all had to be remembered. Visual art forms, such as jewellery-making, left material remnants containing a degree of information that might have allowed for future copying despite a break in lines of information transmission - but the more complex the method of its creation, the less feasible the chance of any previous type of behaviour ever being rejuvenated without a direct observation/copy linkage. This is also true of discarded stone tools, but once more, an increasing level of complexity in production would have resulted in an extremely low - if not impossible - chance of the skill ever being rekindled.

The method of manufacture used in making an artefact is important: if components were transformed during processing - for example when glues were made by mixing several components and then heating them - the identity of the original ingredients would be disguised. Similarly, when making the crayons for cave painting, the binding agents used might have been difficult to determine if that knowledge was lost. Furthermore, during heating processes for glues or pigments, knowledge of the temperatures and their duration, as well as what the correct proportions of each ingredient were, would also have been important information to retain. Whilst a cave painting can provide a lot of information about what it depicts (in the case of a naturalistic representation), the long and drawn out processes involved in its creation are not so apparent.

Lyn Wadley (2010) highlights the difficulties of reverse engineering, when discussing the cognitive demands of complex composite tool manufacture by hominids in South Africa from up to 70,000 years ago. The tools used compound adhesives, and the use of glues and twine to construct them required an incredibly complex set of procedures; the ability to create and use the glue alone required considerable training. Any reverse engineering would have been extremely unlikely. Furthermore, even if an artefact were to be found by later Paleolithic inhabitants, its basic function - let alone its method of manufacture - would probably have been almost impossible to decipher.

\subsection{The fragility of socially transmitted information}

Non-material culture of the Paleolithic, such as the art forms of dance, music, body-painting, and storytelling were transmitted socially between individuals and spread throughout and over 
generations via a process of observation and copying - a process that allowed an accumulation of information through time by being propagated from brain to brain. Similarly, the many day-to-day skills including tool making and weapon use, and even the collection and processing of pigments, would all have had to first be observed and then committed to memory. But if at any point in time there were ever a break, or even a partial blockage in the contiguity of these temporary cranial storage spaces, then any novel or inspirational ideas would have been lost. Breaks in information flow between individuals may still have been overcome via alternative pathways, but if whole populations or generations became informationally separated from each other then any innovative behaviours would have been lost. This danger would have been especially acute in the small, isolated societies that existed in glacial Europe during the Paleolithic - an accidental death, for example, could result in the loss of valuable and potentially life-enhancing skills (Powell, Shennan et al. 2009).

Some might argue that there is an additional information flow to the one above: a biological one. It is certainly true that some traits gradually become ingrained in our genetic code, for selection preserves and propagates those genes that produce the type of phenotypic behaviour that enhance overall fitness. But this process occurs over considerable periods of time - perhaps over hundreds of generations in the human case - and produces generalised rather that specific behavioural patterns. So whilst it might be argued that there was a genetically encoded disposition for early humans to produce certain art forms such as music or dance, these would have been in a very basic form - any artistic innateness would only produce a generic tendency rather than any particular style or fashion. General dispositions are produced genetically, but individual styles are transmitted through culture - and non-material culture had to be passed on from brain to brain.

These different information channels highlight the often precarious nature of maintaining a new type of fitness-enhancing behaviour, and we will never completely know which ones failed to persist and disappeared - possibly due to quirks of fate (although archaeological discoveries have at times revealed some examples). It is of course possible that some forms of a particularly useful behaviour - for example an innovative hunting technique - might have been lost only to be 'reinvented' at a later date (Hovers, Kuhn et al. 2006). But the ability to store information in the environment would make an idea a lot more robust and give it a much better chance of surviving. 


\subsection{Material culture as a store of information}

Unlike non-material culture, parietal and portable art can leave an enduring mark. These artefacts store individual ideas and pieces of cultural information, and leave them out in the environment for all and sundry to assimilate. Most importantly, this information is stored completely independent of a human brain - it is stored in the environment and has the potential to live on until a new brain detects, interprets, and assimilates it once more. Of course, the method of production may be lost, but the representation itself is not - however, if it must be interpreted through local conventions, then some or all of its information will be inaccessible. One reason why we are puzzled by cave art is that we have lost all those conventions, as well as losing most of the art itself. So cave art and other art is only partly independent of the agents who made it, and the initial idea or information only sometimes lives on in its entirety. Nowadays, we take it for granted that we can preserve information in the environment for later retrieval, but as previously discussed, this continuity of information flow was not so easy during the Paleolithic.

I have previously mentioned some of Steven Mithen's views on material culture in chapter 3. To recap, Mithen has argued that the human use of material culture was a critical factor in our cognitive evolution. He believes that it created a positive feedback loop that increased its potential still further; it also enabled the creation of a network of minds that could become partially disembodied. Furthermore, he says that material culture allowed ideas to span long periods of time, and to be passed between people unknown to each other. He also claims that it is "the prime means by which minds are extended out of the body" (Mithen 1998, p. 181) - a notion that I will discuss in detail shortly.

Material culture can, in this way, be viewed as a kind of non-biological memory, and Mithen shows that archaeologists believe that the artwork of the Upper Paleolithic was probably functioning as a store of information (Mithen 1998). Merlin Donald (1991) has investigated this possibility in great depth and also claims that material objects were a form of 'external symbolic storage'. Donald uses the word 'exogram' to describe an external memory, such as an externally stored record of an idea, whilst he calls an internal memory an 'engram' (Donald 1991). Mithen refers to cave art and carvings as being a "remarkable development of culture" (Mithen 1998, p. 10) because they were an important step in the extension of the mind into material culture that provided the possibility for knowledge to accumulate. 
Additionally, Mithen argues that cave art functions "to cue the recall of knowledge about the natural world, and explicitly store some of that knowledge so that it can be transmitted to future generations" (Mithen 1998, p. 182). But I'm not convinced by this explanation. The people of the Paleolithic era were very much in touch with their surrounding world, unlike the more disengaged people of today who decorate their homes with posters and paintings. They would have come into regular contact with most of the species depicted on the cave walls - so why would they have needed such mechanisms to recall this information? Equally, however, survival and reproduction would have been the two main themes dominating their minds, so it might not be so surprising that images linked to hunting and sex would be what they mostly drew. Although people of the Paleolithic were acutely aware of their environments, cave art and the rituals and culture that surrounded it emphasised and reinforced the special nature and mythical importance of the creatures that were central to their survival. Due to the amount of effort that went into a painting, the choice of animal depicted would have indicated how significant it was to the people who drew it - a cave painting stored the subject's importance. The hunt was necessary for food, but it also fostered bonding and improved group coordination. Cave art strengthened the importance of their relationships to their prey and between themselves. So although purely speculation, I believe that cave art was an external information store, but not a field guide; what it did was store social information, or information about what was important to the artists and their community. The implicit information supplied by cave art improved survival on a number of fronts.

\subsection{Epistemically engineering the environment}

As humans increasingly manipulated their environment through their more sophisticated behaviour, they began to leave valuable information there. The interpretation of some of that information, however, was becoming ever more opaque due its growing complexity. This was particularly true of the harsher environments, such as those in glacial Europe. Additionally, the amount of information that brains were required to hold was becoming increasingly more demanding. And as that amount of information grew, humans living in small and isolated groups like the ones in Western Europe faced the risk of permanently losing crucial data and skills. Storing information in the world would have both reduced its susceptibility to being lost, and also alleviated the internal cognitive load being placed upon the brain. Thus, the ability to epistemically engineer the environment may have proved an adaptive advantage.

It has been speculated that although Neanderthals living in Western Europe were capable of surviving in that harsh climate, when anatomically modern humans arrived there, their brains were 
- based upon what has been reported by McBrearty \& Brooks (2000) - more flexible and creative when encountering the additional climatic pressures (Henshilwood and Marean 2003). So the advantages of using the environment as a store of information might have been better exploited by the Cro-Magnons than the Neanderthals. I believe that one of the early technologies used by CroMagnons for externalising the information stored in their brains was cave art. Another example was the creation of portable art in the form of carvings. Other epistemic tools might well have existed, but they have either not (as yet) appeared in the archaeological record, or have not been recognised for their true use. An example could be, for instance, that a piece of wood was used for keeping count of something by placing notches in it. If this was the case, then it is possible that rather than cave art being explicitly for the purpose of putting information out into the world, it may instead have been a by-product of this type of behaviour. But without knowing the true motivations for cave art's production, this notion cannot be pursued any further. What can be said is that early forms of cognitive tools helped to make humans smarter. The use of epistemic engineering would have improved the fidelity of information transfer and made human cognitive systems both more efficient, and more reliable.

Paul Bahn supports the above claims and believes that "the function of most prehistoric art was probably to affect the knowledge or behaviour of the people who could read those messages" (Bahn 1998, p. xvii). He argues that these early paintings were a way of recording and transmitting information, and in this way they were effectively an early form of 'proto-writing'. Both writing and prehistoric art, he says, are methods of documenting information for later retrieval (Bahn 1998). In the painting below from Chauvet cave, not just the animals' representations are recorded: it is also noteworthy for an apparent attempt to portray movement in the case of the top rhino. The occasional paintings of a hunting scene, or animals marked with spears in them, contain even more stored information and would all be considered as proto-recordings. 


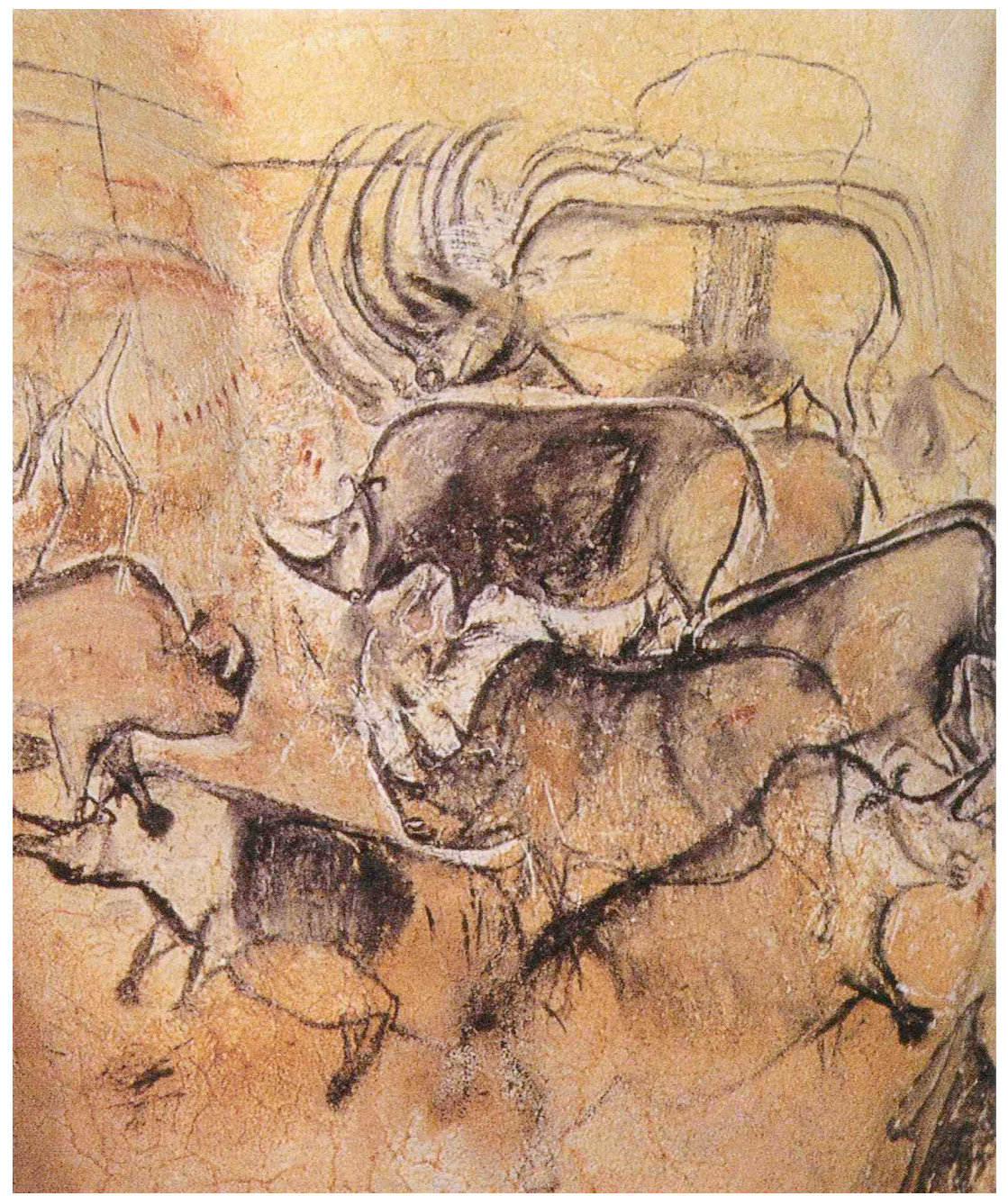

Figure 4.1 Rhinoceri, Chauvet Cave, France - over 30.000 yrs old (White 2003)

The act of creating cave art was a method of putting information stored in a brain out into the world - but how can this best be explained in terms of the brain's cognitive processes? Was cave art either an early form of cognitive extension, or was it an early and important form of epistemic technology? I will now examine two theories that attempt to address this issue: one is the theory of the Extended Mind, whilst the other is the theory of Niche Construction. Extended mind theory makes the claim that at times elements of the environment constitute a part of a human's cognitive system. The theory of niche construction examines how organisms alter their habitat to improve their chances of survival and/or reproductive success; sometimes they deliberately alter it epistemically, in ways that allow current and future generations to scaffold their cognitive powers. 


\section{THE MIND BEYOND THE BODY?}

Intuitively, we would claim that the surrounding environment plays only a passive role in our mind's cognitive operations and that our cognitive processes are contained solely within our brain. Via our perceptual apparatus we encounter the world; our brain receives and then analyses the information that our perceptions provide, and then it initiates appropriate bodily actions. It's a process of perception, followed by cognition, which then results in action. But some argue that this picture of cognition is incorrect and that it should not be strictly limited to the bounds of the body. In 1998, Andy Clark and David Chalmers wrote a paper entitled The Extended Mind in the journal Analysis that put forward their initial claims for this alternative view of human cognition.

Additionally, although one might regard the brain alone as being responsible for all of our cognitive function, it could reasonably be argued that the rest of the body should be included too. The complete nervous system, or the sensorimotor functions, which are comprised of our motor skills and sense organs, could equally be viewed as being part of a human's cognitive apparatus because they also play a role in cognitive processes. For this reason, the theory of 'embodied cognition' claims that the whole of the body is involved in cognition, not just the brain. This theory has it's own set of arguments, but for the purpose of this discussion they do not need to be expanded upon here. ${ }^{17}$ It should be noted that during the following appraisal of the extended mind thesis I will, when discussing a non-extended mind, be implicitly referring to a cognitive system that encapsulates the whole body - everything up until the barrier of the skin. Although this somewhat simplifies the issue, it does at least allow me to make a clear distinction between cognition contained within the body, and anything else that lies outside of it.

\subsection{The Extended Mind Thesis}

In their original paper, Andy Clark and David Chalmers (1998) illustrated their extended mind thesis through an imaginary person, Otto, and his use of a notebook to store information that includes names and addresses. The premise of their theory is that Otto has become so accustomed to using his notebook that he accesses it automatically, just like any part of his mind contained within his head - thus the notebook constitutes a part of his extended mind. This notion, which relies on what has been termed the Parity Principle, makes the claim that the notebook representation and an

17 See Richard Menary's collection of papers in his book The Extended Mind (2010) for an in-depth discussion of this issue. 
internal belief are functionally equivalent in Otto's cognitive system, and I will discuss this issue in further detail shortly.

This seminal paper by Clark \& Chalmers has subsequently sparked much debate. Andy Clark has since gone on to publish numerous papers and books that both defend and expand his and David Chalmers' initial ideas. Richard Menary, in his recent book The Extended Mind (2010), has collated a group of authoritative papers on the subject and provides a nice summary of the Extended Mind thesis in the introduction.

As previously mentioned, the presiding school of thought is that whilst humans may alter or use their environment (as indeed many other animals do), the world that is external to the human body has only a passive influence on our cognition, and hence this theory is referred to as passive externalism. However, Clark \& Chalmers argue the case for active externalism, the converse of this situation. Active externalism claims that sometimes parts of the environment play an active role in human cognitive processes. And Clark \& Chalmers go even further than this, by claiming that at times elements of the environment actually constitute and drive cognitive processes. Their thesis claims that the brain and the environment can be coupled together in a two-way interaction, forming one complete cognitive system - and if any one of these environmental cognitive props were to be removed, then it is claimed that the resulting effect and change in behaviour would be the equivalent of losing a part of the brain along with its particular function (Clark and Chalmers 1998).

According to Clark \& Chalmers, when a human organism and part of its environment are linked together cognitively, they are deemed to have formed what they term a coupled system. Clark \& Chalmers provide an example of one of these coupled systems as a way of better demonstrating their idea: when playing scrabble, moving the tiles is not just a physical action, it is a cognitive act that forms a cognitive loop between the player and the tiles. They argue that the choice of any given word is the result of an extended cognitive process that involved the rearrangement of the tiles; the rearrangement should be seen as part of thought, not of action (Clark and Chalmers 1998).

One of the problems that arise when attempting to partially externalise human cognition, is the issue of coupling. In particular, this is highlighted by the contrast between the inherent coupling of processes of the brain and body, and the far more tenuous coupling that occurs between the brain and the environment. Linked to the issue of coupling is the need for portability. By their very nature, many features of the environment that otherwise have the potential to become a part of Clark \& Chalmers' extended mind are either fixed in location, or are at least not readily movable. 
The immobility of many components of the environment contrasts sharply with the free-flowing portability of the brain and body, which has its cognitive processes contained solely within the bounds of the skin, making it a complete and totally portable cognitive system. In an attempt to preclude this expected attack Clark \& Chalmers argued that to be considered as an external cognitive process, any part of the environment needs only to be coupled reliably.

In his 2003 book Natural Born Cyborgs, Clark expands on the idea of agent-world coupling by discussing the important human cognitive ability of treating a piece of the environment in two completely different ways: in one way we can treat pieces of it as merely independent objects, but in another way we can 'absorb' those same pieces into feeling like they were a part of our body. Clark refers to this trick as a kind of mental 'flippability' and he highlights our use of a hammer or a pen to demonstrate this cognitive skill (Clark 2003). Being able to treat inanimate objects as though they were a part of the body may also have led to humans seamlessly using cognitive tools, because if the brain is capable of assimilating an extension to the physical body, then it might also be capable of extending its cognitive processes.

In contrast to the imaginary Otto and his dependence on the use of a notebook to supplement his poor memory, Clark \& Chalmers also introduced an alternative fictitious person named Inga, who has a good memory and is able to remember names, places, and addresses without the need for external aids such as notebooks. This contrast highlights one of the key requirements of their thesis: the reliance on the theory of functionalism - the claim in philosophy of mind discussions that it is a cognitive system's function, rather than its substance, that is the important issue when analysing it. For their extended mind thesis to be credible it needed to rely on this theory of functionalism. As an example, if an element of a cognitive process contained within the brain can equally perform that same function externally (such as Otto's notebook versus Inga's biological memory), then it can form part of a coupled system resulting in an extended mind.

To assist in determining whether any particular environmental feature could be deemed as being part of a cognitive process, Clark \& Chalmers refer to the parity principle, as previously mentioned. The parity principle is defined as follows:

If, as we confront some task, a part of the world functions as a process which, were it done in the head, we would have no hesitation in recognizing as part of the cognitive process, then that part of the world is (so we claim) part of the cognitive process. (Clark and Chalmers 1998, p. 8) 
Although the parity principle may be used as a guide for whether an external resource can be regarded as a part of a mind, Clark \& Chalmers also provide a set of rules that further distinguish those that can and can not be part of the system. They examine some 'puzzle cases' that challenge our intuitions and list 4 criteria based upon their example of Otto and his notebook. Firstly, the notebook is a constant in Otto's life and he would almost always refer to it, if applicable, before taking any action or making a decision. Secondly the information in the notebook is at any given moment easily accessed. Thirdly, the information is trusted unequivocally. And finally, the information was consciously endorsed at some previous point in time - Otto believes it is true.

In recent times however, the parity principle has been criticised as being too liberal for at least two reasons: firstly, it could be interpreted as being a method of externalising all internal cognitive processes, and secondly, it could also be used for classifying an external process as being cognitive based solely upon its similarity to an internal process (Menary 2010). A cognitive feature such as biological memory clearly has disparate physical properties to its external equivalent, so any claim that the two are directly interchangeable would seem incorrect.

Because of the criticisms associated with the parity principle, Menary and John Sutton (in Menary 2010) report that the defenders of the extended mind thesis have in more recent times shifted their arguments away from the functionality claims that it makes. The new focus is to stress the complementary contributions of external cognitive props. So rather than arguing that what matters for an extended mind is whether an external component (e.g. a notebook) functions the same way as an internal component (e.g. biological memory), the emphasis has instead become one of claiming that if external processes complement internal cognitive processes, then they qualify as being a part of that particular extended mind. The external processes may well have different properties to their internal counterparts, but just as long as they make complementary contributions they can qualify as a component of an extended mind. Sutton calls this new method of evaluating an extended mind the complementarity principle (Menary 2010). Thus, the complementarity principle allows exograms to be radically different from engrams, even when performing in the same process (Menary 2010).

In arguing for the extended mind thesis, Clark \& Chalmers use real-world examples to demonstrate situations where some of the cognitive processing is off-loaded to the world, and thus argue that we use the environment in myriad ways to effectively 'outsource' the cognitive processes of our minds (Clark and Chalmers 1998). They refer to a series of experiments conducted by David Kirsh and Paul Maglio (1994) that involve the playing of a computer game of Tetris, and which examine the ability of players to rotate objects both mentally and physically. During a game, the best players 
made use of the extremes of the screen console when identifying each game piece - something Clark \& Chalmers claim was the formation of a cognitive loop. They also provide everyday examples of the human manipulation of the environment (e.g. the use of a notebook).

The most obvious modern-day case of this cognitive outsourcing is the regular supplementing of our memory by the use of simple aids like notebooks and diaries, through to the utilising of technological aids such as computers and personal digital assistants (PDA's). We also use the environment to help off-load other aspects of cognitive processing, such as when we rearrange the playing cards we have been dealt in order to make it easier to calculate how we should best play the hand, or when we move letters in a scrabble rack to make it easier for our brains to think of words that they can produce. We effectively form and create patterns in the environment to assist our brains based upon the way that they have evolved to process environmental information.

Clark \& Chalmers also discuss how the extended mind fits in with human evolution: "it may be that the biological brain has evolved and matured in ways which factor in the reliable presence of a manipulable external environment" (Clark and Chalmers 1998, p. 11). In particular, they speculate that the brain has evolved to make use of parts of the environment to reduce its memory load, and they refer to how our visual system makes assumptions about the natural scenes it encounters (Clark and Chalmers 1998). These cognitive short cuts are often exploited by magicians' tricks.

So Clark \& Chalmers claim that humans may have evolved to off-load cognitive processes such as memory into the environment and also alter it in ways that allow us to dramatically increase our cognitive powers - the environment is part of a cognitive loop that traverses brain, body, and world (Clark and Chalmers 1998). To support this idea, they suggest that our use of language is possibly one of the main methods of how we extend our cognitive processes out into the world so as to improve our cognitive abilities. Language allows brains to share and solve problems together; it also lets an individual place their thoughts out in the world (e.g. writing on paper), allowing them to be analysed and reinterpreted in a more manageable and orderly way than if they were held solely within the head. They further speculate that language might even have evolved specifically - or at least in part - for the purpose of facilitating an extended mind (Clark and Chalmers 1998).

At this point I will resist any further highlighting of the extended mind theory or criticisms by its opponents. My sole interest here is to evaluate whether cave art was a new way of manipulating the environment to improve human cognitive capabilities, and if the extended mind theory is able to capture this - I therefore remain neutral on the conceptual arguments associated with the theory. I 
will now examine a claim made by Steven Mithen that cave art was an early example of an extended mind.

\subsection{Cave Art and The Extended Mind}

So the theory of the extended mind claims that part of our minds are extended out into the world, beyond the barrier of our skin; the mind is not solely confined to the head, it incorporates parts of the environment as well. In the journal Metascience, Steven Mithen (2004) wrote a review of Andy Clark's 2003 book Natural Born Cyborgs where he elaborated on his extended mind theory. In that review Mithen admitted to realising that his own 1998 book The Prehistory of the Mind had overlooked an important part of our cognitive evolution. Whilst he wrote a favourable review of Clark's book, Mithen had one main criticism: it did not address when the extended mind of humans first began - what were its origins? Mithen then went on to tentatively suggest to Clark what could have been four possible stages of a developing extended mind.

The first stage of an extended mind, says Mithen, would have happened with the development of language, which he claims occurred approximately 500,000 years ago (he argues for this date based upon archaeological evidence of the evolution of the vocal tract). The invention of language would have enabled the sharing of ideas and the networking of minds together, producing a much more complex and flexible behaviour. The possession of language during this period would also explain the evidence of big game hunting and the emergence of the hybrid 'Levallois' flint knapping technique - both of which, he says, would have required a method of communication to convey planned actions (in the case of hunting) or the sharing of different knapping knowledge in developing the latter skill.

According to Mithen, the second stage of an extended mind was brought about by the origin of art (in the form of cave art and carvings) approximately 100,000 years ago (and I will discuss this stage in more detail shortly). The third stage was associated with the origin of sedentism roughly 10,000 years ago, which meant that for the first time in their history human brains were permanently surrounded by material culture. The fourth and final stage of an extended mind was brought about by the origin of writing, which occurred roughly 3000 BC in Mesopotamia; writing allowed the separate powers of material culture and language to be combined, thus ramping up the potential of both to further develop the modern human mind. 
Of these proposed four stages, Mithen argues that the origin of cave art and carvings was the key development for an extended mind, "it was a time when culture became as important as biology for human thought" (Mithen 2004, p. 167). Prior to the cave art era, ideas or thoughts could have been exchanged via language but they would have remained within the heads of all those who encountered them. The arrival of the ability to produce these parietal and portable art forms immediately enabled the possibility of 'freezing' an individual thought or idea out in the world for all to appreciate. An idea could now spread beyond earshot to other, non-present minds, and also to later generations, devoid of the risk of it being corrupted or lost. Most importantly, it allowed thoughts to be re-interpreted - as Mithen puts it, it opened "the floodgates of self-reflective reason" (Mithen 2004, p. 167). I will discuss this last point again in this chapter's conclusion.

Responding to Mithen's review in the same edition of Metascience, Andy Clark (2004) immediately accepted the claim that material culture has played a critical role in our cognitive advancement and agrees that pinning down its key developments is an important issue that needs to be addressed. Like Mithen, Clark also admits to wondering whether it was something other than the regularly touted advent of language that triggered the most significant advance in human cognitive development; he is therefore excited by Mithen's suggestion that it was instead art and its ability to 'freeze' thoughts. So, molding his extended mind thesis onto Mithen's idea, Clark finds it "compelling" to think that the beginning of art some 100,000 years ago "marked the moment when we humans first began to actively extend, manipulate, and augment our own minds" (Clark 2004, p. 176). He further argues that art might well have been "the early signature of cognition-enhancing technology" (Clark 2004, p. 176).

\subsection{Is Mithen right?}

Prima facie, cave art appears to be an early example of an epistemic tool; it seems to be a clear attempt by the brain to off-load some of its resources out into its environment. But can cave art really be seen as one of the first stages in the development of an extended mind as Steven Mithen has claimed? The best way to answer this question is to evaluate cave art's attributes and compare them with the main tenets of the extended mind thesis - it should then be possible to ascertain whether they might - or might not - apply to cave art.

When applying Clark \& Chalmers' Parity Principle to cave art, it seems that as a method of storing of internal representations externally it satisfies this initial test. From a functionalist's point of view, it could be claimed that cave art has a reasonable symmetry with biological memory; it reliably 
stores information out in the environment for later retrieval. Cave art is a combination of creating a representation in the head and then reproducing (and storing) that image physically in the environment, rather than biological memory.

But satisfying the parity principle alone is not sufficient. A second condition for qualifying as part of an extended mind is that a piece of the environment has to be readily available for coupling. Cave art however, in the form of parietal paintings and engravings, does not comply with this requirement because it is not so easily accessed. So whilst cave art appears to be functionally akin to biological memory, due to its lack of ready accessibility it has different qualities. But this is more applicable to the cave paintings that were in the deeper parts of the caves. Any Paleolithic drawings that were either near a cave's entrance, or out in the open - but have since been destroyed - would have been much more accessible, and thus more easily coupled, but they were still dissimilar to biological memory.

However, as explained above, a number of criticisms have been levelled at the parity principle, and the complementarity principle is now the preferred method of appraising potential components of an extended mind. The external representations of cave art are more stable than internal biological memories, and so allow for better cognitive scaffolding. More importantly, cave art also complements internal cognitive processes by allowing thoughts and ideas to be reinterpreted via our visual apparatus. So it would appear that these methods of representation satisfy the complementarity principle. But it is not obvious either way from Menary's discussion whether complementary representations need to be readily available. So cave art reveals an ambiguity in the concept of complementary coupling - is it enough to know where the art is, so you can consult it as needed?

An extended mind also requires portability. By their very nature, many features of the environment that have the potential to become a part of an extended mind are either completely fixed in location, or are at least not readily movable. This is certainly true of cave art; it is physically immobile and therefore fails the test for portability. The small, painted plaquettes, however, which were probably used for refining or honing the artist's skills, do to some extent fulfil the requirement for portability, and thus reliable coupling; they would have been readily available as and when required. The Paleolithic carvings such as Venus figurines, which were relatively light and mobile, could also travel with an individual. 
As a final examination of whether cave art fits the extended mind model, we can review the four criteria listed by Clark \& Chalmers in their original paper. Firstly, cave art is required to have been a constant in the life of a Cro-Magnon, but due to its remote nature this would not have been the case. The evidence of a lack of footprints in the caves supports this, and so the paintings would not have been referred to on a regular basis before actions were taken. Accessibility was another requirement specified by Clark \& Chalmers but as we have seen, apart from the paintings close to cave entrances, most would have been difficult to reach. An element of planning was also needed before venturing into a cave to view the paintings, with the gathering of lighting equipment and sufficient fuel for the journey an obvious first step - so accessibility was limited. Of the final two requirements, we do not know whether Cro-Magnons would have either consciously endorsed the paintings or trusted them unequivocally.

So the static nature of cave art restricts its ability to be seen as a component of an extended mind, but the mobility of carvings and plaquettes provide a much greater potential for being tied to a human's cognitive processes. The plaquettes, and even more so the portable carvings, are somewhat similar to Otto's notebook - they can be carried on one's person and are thus readily available for reliable coupling. To summarise, different forms of cave art are integrated into cognitive performance in different ways. And it is unlikely that any, or many examples, clearly meet the Clark \& Chalmers criteria for being part of the system. Due to its lack of portability and fixed location, cave art was more like a library for Otto than his notebook.

So whilst there is much ongoing debate surrounding the extended mind thesis, I do not believe that Paleolithic cave paintings can be regarded as being components of extended minds. Both cave art and carvings were, I believe, a case of passive externalism - they may have had an input to our ancestors' cognitive processes, but they did not constitute a part of them. I think that cave art was much more likely to have been an early example of manipulating the environment epistemically. This method of storing information and ideas allowed them to be spread and reinterpreted by either the artists themselves, or by others.

The use of material culture, such as cave art, has allowed for the ramping up of cognitive power by the process of cultural scaffolding. In the next section I examine an alternative method to the extended mind theory for appraising this type of information storage. 


\section{AN ALTERNATIVE VIEW}

Ignoring any cultural meaning, what we can say the creation of cave art did was that it altered a part of the environment of the Upper Paleolithic. But it did more than just physically alter it; it also introduced information into the environment that could subsequently be assimilated by anyone who encountered it - not just the artist. I will now review niche construction theory, which is a method of analysing the effects produced when organisms alter their environments. I will then discuss how this theory can account for the behaviour that produced the cave art of the Upper Paleolithic in Western Europe.

\subsection{Niche Construction theory}

Niche construction theory is a relatively recent development in evolutionary biology, and I will be referring to the 2003 book Niche Construction by Odling-Smee, Laland et al. when presenting the following summary of its basic principles.

During the course of their lifetime, organisms alter their surrounding environment as a by-product of their existence by using and redistributing its resources; they further alter it during their subsequent demise. Some organisms also rearrange it deliberately by modifying their habitat: birds create nests, some animals make their own burrows, and spiders create webs. These changes can have an impact not only upon the organisms themselves, but also on other organisms that encounter or share that same living space. This modification of the environment has been termed niche construction. Additionally, once a niche exists, its effects can persist quite separately from the organism that created it, sometimes for many years afterwards. Niche construction is often linked to the effects produced by whole populations, not just solitary individuals. Furthermore, it has the potential to affect entire ecosystems.

Proponents of niche construction theory argue that the effects of these alterations are sometimes so significant that they can modify selective environments (Odling-Smee, Laland et al. 2003). A good example of this is how earthworms enrich the soil in which they live by both aerating it and converting dead organic matter into rich nutrients via excreted casts - and this improved soil fertility then promotes increased plant growth. So the construction of a niche can affect the selection pressures operating on not only an organism and its population, but also on those of other organisms. Niche construction can therefore play an important role in evolution, but only if the 
effects impact the same local environment, and persist for a long enough period of time - a transitory effect will not be sufficient (Odling-Smee, Laland et al. 2003). The effects may be continuous, cumulative, or reliably repeated by each subsequent generation as a result of inherited genes that express themselves in the same way (e.g. spiders creating webs). Through this mechanism it is therefore argued that niche construction can have evolutionary, as well as ecological consequences.

Additionally, this ecological engineering can, over time, result in a process of cumulative niche construction whereby it affects the selective pressures operating not only on the present, but also the succeeding generations. A regularly used example of this type of niche construction, which was first suggested by Richard Dawkins (1982), is that of a beaver's dam. A beaver's dam improves its chances of survival by being both a refuge from predators, and by providing improved access to food. But it may also be inherited later on by its offspring, and the original dam may be improved upon and strengthened over time by those subsequent generations. A beaver can therefore create an ecological inheritance. Importantly then, offspring not only inherit their parents' genes, but they can sometimes inherent an engineered environment of changed selection pressures.

At this point it is also worth noting that whilst a genetic inheritance includes information in the form of a set of instructions within their DNA, an ecological inheritance is typically a physical rather than informational process (Odling-Smee, Laland et al. 2003). But humans are different in this respect, and I will be discussing this shortly. An additional difference between genetic and ecological inheritance is the way it is transmitted. A genetic inheritance is solely a vertical, downstream process between parent and offspring. But an ecological inheritance can also be passed horizontally and obliquely, so that whole populations, as well as different organisms can benefit. They can also produce feedback into the ecosystem by making further alterations to that particular niche. Changes can therefore be much more dynamic and rapid than their genetic equivalent.

Niche construction's ability to modify selection pressures over prolonged periods of time produces the strongest claim made by this theory: that an organism's evolution not only depends on natural selection, but also on niche construction. An organism's evolution is influenced by the way it adapts to its environment, but also by how it continues to alter that environment in order to improve its fitness. In this way, there follows an ongoing process of two-way interaction between environment and organism; niche construction "introduces feedback into the evolutionary dynamic" (OdlingSmee, Laland et al. 2003, p. 2). An example of this is the defecation habits of burrowing animals: over time, burrows gradually become polluted and uninhabitable through the build up of excrement. 
Natural selection would favour those animals that place their faeces outside of the burrow because the animals with the cleaner (and therefore longer-lasting) burrows will have a fitness advantage.

Another important aspect of niche construction is social organisation (Sterelny 2003). A group that cooperates together is often much more effective (and safer) than individuals who operate alone for example, schooling fish make life much more difficult for predators. An even more advanced form of cooperation is demonstrated by honey bees: they not only create a nest, but they also collectively regulate its temperature throughout the year and store honey so that they can remain active through the winter (Odling-Smee, Laland et al. 2003). Apart from protecting them against climate extremes, this also provides ideal conditions for offspring to develop (Laland and Brown 2006).

So organisms make use of their environment by manipulating it in a variety of ways. But as I have previously mentioned, the world is informationally rich and those individuals who make the best use of this information are better able to negotiate and prosper in it. Plants use day length to trigger their seasonal variation; bird migrations are triggered by seasonal changes, but they then use the earth's magnetic field and the position of the sun for navigation (Sterelny 2003). Some organisms not only use their environment's information, they also change it through a process of epistemic engineering. In this way it is possible to either hide or emphasise certain pieces of environmental information. Examples of this practice are the camouflaging of burrows or nests to protect against predators, or how bowerbirds decorate their nests with shiny objects to attract mates. Others mark their territory with scent (Sterelny 2003).

\subsection{Human Niche Construction}

Humans are incredibly sophisticated in their manipulation of the environment. Tool use in particular required a great understanding of materials and how they could be transformed. By creating tools, and then using them, humans were able to engineer their worlds even further. Other animals are also capable of tool use, but they only use them to solve an immediate or specific problem (Sterelny 2003); humans use tools much more creatively and are able to apply them to new problems.

The use of niche construction has seen humans modify selection pressures in many ways to improve their overall fitness. This has also affected their evolution. As an example, humans have chosen the least aggressive and most productive individuals of a species when domesticating them, which has 
resulted in breeds of animals that are vastly different from their wild cousins. Humans have in turn adapted to the food supply provided by these animals, for instance by becoming lactose tolerant (Laland and Brown 2002).

Humans are particularly good at providing an ecological inheritance for their offspring. Human infants depend on their parents nurturing them for a much longer period of time than any other animal, and humans have modified their environment to not only provide a high degree of protection for their offspring, but also to enhance an offspring's learning processes. This appears to have resulted in a process of co-evolution: humans have adapted to the availability of this kind of 'knowledge incubator' by maturing at a slower rate than other primates in order to allow for this extended period of skill acquisition (Sterelny 2003). And this brings us to the issue of epistemic inheritance.

The social environment created by humans is both advanced and complex. The high degree of cooperation between humans has allowed them to gain access to parts of their environment that would have been otherwise impossible. Hunting large animals during the Paleolithic is one such example. But humans also make good use of the information in their environmental, not just its physical resources. Being able to track an animal's footprints during the aforementioned hunt is just one example of this. The use of environmental information, combined with their sociability, has allowed humans to share what they learn and scaffold it, producing a cumulative epistemic niche. All of this information is subsequently passed on to offspring in the form of an epistemic inheritance.

Cultural transmission through social learning is believed to have played a major role in the development of human cognitive skills (Tomasello 1999). Additionally, it is claimed that the ability to manipulate the environment to both assist learning and also to transmit accumulating amounts of scaffolded information to succeeding generations (through the use of language, tools, and material culture), lies behind the intelligent behaviour, and superior intelligence of humans (Tomasello 1999; Sterelny 2003). It is this inheriting of accumulated information, not just a physically altered environment, that sets humans apart from other animals. Furthermore, as Tomasello points out, social or cultural transmission "works on time scales many orders of magnitude faster than those of organic evolution" (Tomasello 1999, p. 4). He also refers to this unique human accumulation of skills and cultural information as the "ratchet effect" (Tomasello 1999). 
So, in addition to ecological engineering, humans also alter their informational environment through epistemic engineering. They create a cultural niche via which adaptive information can be transmitted and improved upon over subsequent generations. But with this growing amount of information there would have come an ever-increasing cognitive load.

\subsection{Cave Art and Niche Construction}

I believe that the act of creating cave art is a form of niche construction. I think that this early parietal art was an important milestone in a new use of the environment. It was a part of an accumulating epistemic niche that allowed successive generations to grow smarter by making smarter choices. I will now evaluate the specifics of cave art and attempt to connect them with the specifics of niche construction.

The first part of niche construction theory addresses the issue of environmental engineering. Producing cave art involved deliberately manipulating and redistributing various materials within the environment. It also required an understanding of how those materials could be combined and transformed. The act of creating a cave painting changed the environment in a relatively permanent way, and was more akin to the creation of a bird's nest than a transient spider's web. It was an enduring change that could persist quite separately from its creator and could also be utilised by other individuals.

It is difficult to say exactly how much cave art modified the selection pressures of other organisms; most of the materials used were inert (e.g. the pigment and charcoal), and so their redistribution was unlikely to have affected other organisms. However, the use of fat for lamps would have removed a portion of a human's food source, and so conceivably required hunting more animals. If blood was the binding agent of choice for crayon manufacture, then this would have also created an additional demand for animal carcasses - but unless the quantities involved were considerable, it might have had only a limited impact on hunted animal populations. It is certainly true that if the practice of creating representations on the outside of caves was extensive, then the effects of this type of behaviour may well have been much greater than that of cave art alone. An additional effect may have been the displacing of other animals from the caves and rock shelters used for painting, but the actual impact will never be known.

An ecological inheritance is an important fitness advantage for many animals, but cave art does not appear to offer any physical benefits to humans. What cave art did do, however, was to act as a 
technology for storing information. So rather than being an ecological inheritance, cave art bequeathed an epistemic inheritance. The ability to pass information from generation to generation in a cumulative way has allowed humans to scaffold their knowledge and skills, and the use of language has dramatically improved this process. But cave art was one of the early technologies for retaining and transmitting this information with a higher degree of fidelity. Furthermore, the information contained in cave paintings does not reside within human brains and is therefore much more stable.

This epistemic inheritance also exhibited signs of being cumulative, even though the skills and techniques themselves do not appear to have changed during the period of cave art production. As explained in chapter 1 , the amount of information contained within a cave painting was not always fixed - it sometimes accumulated. Some drawings were created over very long periods - hundreds, or even thousands of years; some were created in stages, with additions gradually being made to existing paintings, whilst others were simply painted over the top of older ones (Clottes and LewisWilliams 1998).

Cave art lasted for perhaps 25,000 years, and thus satisfies a niche construction's requirement of being an enduring presence. According to this theory then, it could have played a role in natural selection. But as I have just stated, its influence on the local environment's selection pressures may have been minimal. It is therefore doubtful whether it played any part in the adaptations of the organisms inhabiting that same ecosystem. Cave art probably did, however, affect the selection pressures that were acting upon humans.

Humans were beginning to use their environment to store information. This type of behaviour would have been beneficial and thus selected for: the action of moving information from the brain to the environment was a method of reducing cognitive load. And as I have already discussed, the amount of information needing to be retained by humans was continuing to grow throughout the Paleolithic and the increasing cognitive load associated with it would have produced selective pressures on humans. Brains capable of utilising technologies for reducing this load would have been at an advantage. The ability to produce cave paintings was one of those technologies. This type of behaviour may also have brought additional (e.g. sexual) benefits as a by-product of participating in this activity, and these would have added to the already present selection pressures - but we will probably never know if this was the case. But the cognitive benefits of cave painting and associated technologies are clear. 
Cave art was also made possible by the existence of the human social niche, which it in turn reinforced. The production of cave paintings would have needed many collaborative acts and a high degree of social organisation: from the collection of all the materials, through to the painting of the larger compositions. An effective human social niche facilitated the large amount of cooperation required for this type of behaviour to occur. It also enabled the artists to acquire the necessary skills for creating the paintings.

\subsection{Summary}

Just as we use tools such as hammers and spades to enhance our physical abilities, so too do we use epistemic tools to manipulate our epistemic environment. The use of epistemic tools improves and supplements - but does not replace - our cognitive abilities. I believe that cave art was an early example of externalising the brain's information, and once embedded in the environment that information could be shared and scaffolded by others. Furthermore, cave art provided a highfidelity flow of information between individuals, as well as generations, by allowing its representations to be passed on unadulterated.

When evaluating cave art's cognitive significance, I believe that niche construction theory provides a better explanation of what it might have been indicating than the extended mind thesis. One immediate advantage that niche construction has is that it is neutral on the issue of functionalism. So when applying niche construction theory to cave art one does not also need to make controversial claims about the philosophy of mind. Put simply, cave art placed information out into the world, how the mind fits in with this is a separate issue.

As a method of modelling human cognition with respect to the epistemic use of the environment, Kim Sterelny $(2004 ; 2010)$ has argued that niche construction theory does this more accurately than the extended mind thesis. Sterelny claims that the human use of epistemic tools should be seen as more of a group than an individual activity - it allowed an idea to become accessible to all and sundry for them to grasp and develop (both immediately and in the future by subsequent generations). Equally, cave art - but even more so the now long gone external paintings - would have been accessible to many, not just the artist. Paleolithic drawings were not the equivalent of someone's personalised private notebook. Cave art extends human cognition, not by forming a part of a human's cognitive system, but instead by being a crucial environmental support that amplifies the capacities of body-bound systems. 


\section{CHAPTER SUMMARY}

Early human tools such as handaxes when used as templates perform the function of storing information in the environment, but this was not their primary role, they were for physical rather than epistemic purposes. Epistemic tools are specifically made for the storing of information out in the environment rather than letting it reside solely inside the head. I believe that cave art was an early example of a specialised epistemic tool and might have been the beginning of purpose-built information technology. Creating pictorial representations in the form of cave art and carvings was not simply an example of off-loading memory into the environment, it was much more than that, it was off-loading an idea. Memory is often very personal and relies on individual perceptual interpretation, but an idea can be interpreted differently and can be developed by others. For instance, tying a knot in one's handkerchief does not in itself provide specific information, but it does act as a mental cue which triggers the brain to ask itself "there was something I had to remember to do, now what was it...?" - but that same knot would mean nothing to someone else.

Cave art was an advancement on the use of language, which could only spread information within earshot. Cave art enabled information to be stored out in the environment long before written language was invented. Humans are highly social animals and one of the reasons why we have been so successful as a species is because we are good at copying and learning from each other and doing this involves the sharing of our information. The joint use of epistemic tools would have been an important advance in facilitating this. It allowed cognitive scaffolding, and cultural changes to develop. It could be argued that both cave art and carvings enhance the creative abilities of the mind by allowing its internal representations to be placed out into the world for additional mental manipulation by allowing the senses to reinterpret them. The possibility of having thoughts about thoughts was suddenly made available to those who encountered this art - and this might have been the cognitive development that led to self-consciousness. ${ }^{18}$

So whilst I agree with Mithen's claim that cave art (and carvings) were a way of 'freezing a thought', I do not believe this was an early case of an 'extended mind' - what cave art facilitated was to make an idea accessible to many, to be shared and expanded on. It seems like much more of a case of epistemic niche construction. According to Sterelny (and I agree), our use of epistemic artefacts should be seen as a social, rather than individual advance - and it is this that has allowed

18 Higher-order thought (HOT) theories of consciousness are based upon this cognitive ability. 
us to gain the intelligence that we now possess (Sterelny 2004). He argues that the extended mind project is really only addressing how a single mind functions, but Sterelny is more interested in examining how minds work together: we share information, including epistemic artefacts which we do not always use selfishly, monopolistically protecting it (Sterelny 2004).

Many organisms participate in epistemic niche construction. Some animals mark their territory with urine, leaving information for others to take notice of. Humans do something similar but in far more sophisticated manner: we erect signs that might contain many details for marking (say) the edge of a plot of land. We can use written language to pass on much more information than any spray of urine could ever manage, but there are similarities. I would not claim that a dog has extended its mind onto some tree, yet it has done a similar thing to what we humans do: information has been placed out in the world deliberately. The environment is full of information, but much of it is neutral and each brain interprets it in own particular way. The act of deliberately altering an organism's world to pass information to members of either the same or different species is, I believe, a major evolutionary step. I think that it is an attempt by a brain to reach out beyond its body, but not only into its environment (for that is just a conduit), I would argue that it is actually attempting to reach out to other brains. Cave art was a primitive form of communication. Just as the Internet has allowed many computers to interconnect, cave art was one step in the growing ability to network minds together.

As human lives became ever more complex, the cognitive load on the brain increased. By using the environment to off-load some of it would have allowed the increase in complexity to continue by reducing internal cognitive load. Cave art was a method of transferring information from the brain into the world, resulting in reduced internal cognitive load and hence costs. It also improved the fidelity of information transfer. Most importantly, cave art began a process of allowing minds to share information and ideas without the need for verbal communication. 


\section{CONCLUSION}

I began this thesis by looking into the background of cave art: in particular, paintings of astonishing quality from up to 40,000 years ago have been found in Western Europe. I have shown however, that the creation of pictorial representations has existed for perhaps up to 100,000 years ago with some of the oldest found so far being discovered in southern Africa - so claims of a 'creative explosion' occurring once anatomically modern humans arrived in Europe are misplaced; they were capable of such cognitive feats well before they arrived in Western Europe, and it seems much more likely that it was a gradually improving process of technical improvement.

The motivations for creating cave art have been debated at length, with many possible scenarios having been put forward. Some claim that this type of behaviour was a by-product of evolution, whilst others have argued that it had evolutionary benefits that clearly demonstrate it was the result of selection pressures. Whether the artists benefitted sexually, thus improving their reproductive fitness, or whether it helped adolescents train their minds to make them better prepared to face the adult world is an open question. Could this type of behaviour be solely for aesthetic reasons and used only for idling away one's leisure time for pleasurable purposes? Or maybe it was part of mysterious pseudo-religious ceremonies resulting in no survival or reproductive advantages, as others have claimed. For all the insightful and interesting discussion about cave art's motivations, we are probably never likely to unravel the apparently mysterious reasons behind its production.

Although there are many questions surrounding the advent of cave art, we can deduce with greater certainty what was required for its production. Creating a sublime pictorial representation on a cave wall required a whole range of cognitive competences - and well before the image was even made there were many actions to plan and prepare. Materials to paint with, tools for applying the colours and creating the lighting, as well as the need to choose a location, were all necessary. These combined skills required the possession of a diverse set of cognitive abilities and I attempted to highlight many of the basic brain skills. I then evaluated what higher-level skills might have been made possible with the possession of these basic cognitive tools.

Whatever motivation lay behind the production of cave art, it appears to be a radically different type of behaviour to anything previously seen. So I next examined whether it could be indicating some other as yet unexplored cognitive breakthrough. The theory of an extended mind, as originally 
suggested by Andy Clark and David Chalmers, was proposed as an explanation for humans making epistemic use of their environment by incorporating it as a part of their mind's cognitive processes. Additionally, Steven Mithen has claimed that the cave art (and carvings) of the Paleolithic, were an early stage in the development of an extended mind. After careful analysis, I believe that the act of altering the surrounding environment in a way that stores information externally from the body, appears to be much better explained by the theory of niche construction. Epistemically engineering the world occupied by humans allows information and ideas to be shared and then scaffolded upon; together with an improved cognitive skill set, activities such as cave art laid the foundations for the eventual information-rich society in which we live in today. Epistemic engineering may even be linked to the cognitive development that led to human self-consciousness. 


\section{REFERENCES}

Bahn, Paul G. (1998). The Cambridge illustrated history of prehistoric art. Cambridge, U.K., New York : Cambridge University Press.

Balter, Michael (2009). "ORIGINS: On the Origin of Art and Symbolism." Science 323(5915): 709-711.

Boyd, Brian (2009). On the origin of stories : evolution, cognition, and fiction. Cambridge, Mass., Belknap Press of Harvard University Press.

Brown, Kyle S., Curtis W. Marean, et al. (2009). "Fire As an Engineering Tool of Early Modern Humans." Science 325(5942): 859-862.

Chauvet, Jean-Marie, Eliette Brunel Deschamps, et al. (1996). Dawn of art : the Chauvet Cave : the oldest known paintings in the world. New York, H.N. Abrams.

Clark, Andy (2003). Natural-born cyborgs : minds, technologies, and the future of human intelligence. Oxford ; New York, Oxford University Press.

Clark, Andy (2004). "We Have Always Been . . Cyborgs." Metascience 13(2): 169-180.

Clark, Andy and David Chalmers (1998). "The Extended Mind." Analysis 58(1): 7-19.

Clottes, Jean (2003). Return to Chauvet Cave : excavating the birthplace of art : the first full report. London, Thames \& Hudson.

Clottes, Jean (2008). Cave art. London ; New York, Phaidon Press.

Clottes, Jean and J. David Lewis-Williams (1998). The Shamans of prehistory : trance and magic in the painted caves. New York, Harry N. Abrams.

Conard, Nicholas J. (2009). "A female figurine from the basal Aurignacian of Hohle Fels Cave in southwestern Germany." Nature 459(7244): 248-252.

Conard, Nicholas J., Maria Malina, et al. (2009). "New flutes document the earliest musical tradition in southwestern Germany." Nature 460(7256): 737-740.

Currie, Gregory and Ian Ravenscroft (2002). Recreative minds : imagination in philosophy and psychology. New York, Oxford University Press.

D'Errico, Francesco (2003). "The invisible frontier. A multiple species model for the origin of behavioral modernity." Evolutionary Anthropology: Issues, News, and Reviews 12(4): 188-202.

D'Errico, Francesco, Paola Villa, et al. (1998). "A Middle Palaeolithic origin of music? Using cavebear bone accumulations to assess the Divje Babe I bone 'flute'." Antiquity 72(275): p65(15).

Dawkins, Richard (1982). The extended phenotype : the gene as the unit of selection. Oxford, U.K., Freeman. 
Deacon, Terrence William (1997). The symbolic species : the co-evolution of language and the brain. New York, W.W. Norton.

Dennett, Daniel Clement (1995). Darwin's dangerous idea : evolution and the meanings of life. New York, Simon \& Schuster.

Dennett, Daniel Clement (1996). Kinds of minds : toward an understanding of consciousness. New York, Basic Books.

Dennett, Daniel Clement (1998). Brainchildren : essays on designing minds. Cambridge, Mass., MIT Press.

Donald, Merlin (1991). Origins of the modern mind : three stages in the evolution of culture and cognition. Cambridge, Mass., Harvard University Press.

Dunbar, R. I. M., Chris Knight, et al. (1999). The evolution of culture : an interdisciplinary view. Edinburgh, Edinburgh University Press.

Dutton, Denis (2009). The art instinct : beauty, pleasure, \& human evolution. New York, Bloomsbury Press.

Fox, Douglas S. (2002). "The Inner Savant." Discover 23(2): 44-49.

Guthrie, R. Dale (2005). The nature of Paleolithic art. Chicago, University of Chicago Press.

Henshilwood, Christopher, Francesco d'Errico, et al. (2004). "Middle Stone Age Shell Beads from South Africa." Science 304(5669): 404.

Henshilwood, Christopher S., Francesco d'Errico, et al. (2009). "Engraved ochres from the Middle Stone Age levels at Blombos Cave, South Africa." Journal of Human Evolution 57(1): 27-47.

Henshilwood, Christopher S., Francesco d'Errico, et al. (2002). "Emergence of Modern Human Behavior: Middle Stone Age Engravings from South Africa." Science 295(5558): 1278-1280.

Henshilwood, Christopher S. and Curtis W. Marean (2003). "The Origin of Modern Human Behavior: Critique of the Models and Their Test Implications." Current Anthropology 44(5): 627651.

Hovers, Erella, Shimon Ilani, et al. (2003). "An Early Case of Color Symbolism: Ochre Use by Modern Humans in Qafzeh Cave." Current Anthropology 44(4): 491-522.

Hovers, Erella, Steven L. Kuhn, et al. (2006). "Now You See it, Now You Don't"-Modern Human Behavior in the Middle Paleolithic. Transitions Before the Transition. M. A. Jochim and R. S. Dickens, Springer US: 295-304.

Humphrey, Nicholas (1998). "Cave Art, Autism, and the Evolution of the Human Mind." Cambridge Archaeological Journal 8(02): 165-191.

Kirsh, David and Paul Maglio (1994). "On distinguishing epistemic from pragmatic action." Cognitive Science 18(4): 513-549. 
Kohn, Marek and Steven Mithen (1999). "Handaxes: products of sexual selection?" Antiquity 73(281): 518.

Laland, Kevin N. and Gillian R. Brown (2002). Sense and nonsense : evolutionary perspectives on human behaviour. Oxford; New York, Oxford University Press.

Laland, Kevin N. and Gillian R. Brown (2006). "Niche construction, human behavior, and the adaptive-lag hypothesis." Evolutionary Anthropology: Issues, News, and Reviews 15(3): 95-104.

Leroi-Gourhan, André (1982). The dawn of European art : an introduction to Palaeolithic cave painting. Cambridge [Cambridgeshire] ; New York, Cambridge University Press.

Lewin, Roger (1998). Principles of human evolution : a core textbook. Malden, Mass., Blackwell Science.

Lewis-Williams, J. David (1983). The rock art of southern Africa. Cambridge [Cambridgeshire] ; New York, Cambridge University Press.

Lewis-Williams, J. David (2002). The mind in the cave : consciousness and the origins of art. London, Thames \& Hudson.

McBrearty, Sally and Alison S. Brooks (2000). "The revolution that wasn't: a new interpretation of the origin of modern human behavior." Journal of Human Evolution 39(5): 453-563.

Menary, Richard (2010). The extended mind. Cambridge, Mass., MIT Press.

Mithen, Steven (2004). "We Have Always Been .. . Cyborgs." Metascience 13(2): 163-169.

Mithen, Steven J. (1996). The prehistory of the mind : a search for the origins of art, religion, and science. London, Thames and Hudson.

Mithen, Steven J. (1998). Creativity in human evolution and prehistory. London ; New York, Routledge.

Mithen, Steven J. (2005). The singing Neanderthals : the origins of music, language, mind and body. London, Weidenfeld \& Nicolson.

Odling-Smee, F. John, Kevin N. Laland, et al. (2003). Niche construction : the neglected process in evolution. Princeton, Princeton University Press.

Pettitt, Paul, Paul G. Bahn, et al. (2007). Palaeolithic cave art at Creswell Crags in European context. Oxford ; New York, Oxford University Press.

Pike, Alistair (2008). "New dating method sheds light on cave art." Planet Earth online. Retrieved 2 July 2010, from http://planetearth.nerc.ac.uk/news/story.aspx?id=186.

Pinker, Steven (1997). How the mind works. New York, Norton.

Powell, Adam, Stephen Shennan, et al. (2009). "Late Pleistocene Demography and the Appearance of Modern Human Behavior." Science 324(5932): 1298-1301.

Ruspoli, Mario (1987). The cave of Lascaux : the final photographs. New York, Abrams. 
Shaw-Williams, Kim (forthcoming). The Relevance of Track-ways Reading (TWR) to the Evolution of Human Cognition, Culture and Communication. MA thesis. Philosophy, Wellington, New Zealand, Victoria University.

Spivey, Nigel Jonathan (2005). How art made the world : a journey to the origins of human creativity. New York, Basic Books.

Sterelny, Kim (2003). Thought in a hostile world : the evolution of human cognition. Malden, MA, Blackwell Pub.

Sterelny, Kim (2004). Externalism, Epistemic Artefacts and the Extended Mind. In R. Schantz (ed.), The Externalist Challenge. Berlin ; New York, Walter de Gruyter: 239-255.

Sterelny, Kim (2010) Minds: extended or scaffolded? Phenomenology and the Cognitive Sciences DOI: $10.1007 / \mathrm{s} 11097-010-9174-\mathrm{y}$

Stiner, Mary and Steven Kuhn (2006). "Changes in the 'Connectedness' and Resilience of Paleolithic Societies in Mediterranean Ecosystems." Human Ecology 34(5): 693-712.

Tomasello, Michael (1999). The cultural origins of human cognition. Cambridge, Mass., Harvard University Press.

Wadley, Lyn (2010). "Compound Adhesive Manufacture as a Behavioral Proxy for Complex Cognition in the Middle Stone Age." Current Anthropology 51(S1): S111-S119.

White, Randall (2003). Prehistoric art : the symbolic journey of humankind. New York, Harry N. Abrams.

Zilhão, João, Diego E. Angelucci, et al. (2010). "Symbolic use of marine shells and mineral pigments by Iberian Neandertals." Proceedings of the National Academy of Sciences 107(3): 10231028. 